\title{
AMALGAMS DETERMINED BY LOCALLY PROJECTIVE ACTIONS
}

\author{
A. A. IVANOV AND S. V. SHPECTOROV
}

\begin{abstract}
A locally projective amalgam is formed by the stabilizer $G(x)$ of a vertex $x$ and the global stabilizer $G\{x, y\}$ of an edge (containing $x$ ) in a group $G$, acting faithfully and locally finitely on a connected graph $\Gamma$ of valency $2^{n}-1$ so that (i) the action is 2-arc-transitive; (ii) the subconstituent $G(x)^{\Gamma(x)}$ is the linear group $S L_{n}(2) \cong L_{n}(2)$ in its natural doubly transitive action and (iii) $[t, G\{x, y\}] \leq O_{2}(G(x) \cap G\{x, y\})$ for some $t \in G\{x, y\} \backslash G(x)$. D. Ž. Djoković and G. L. Miller [DM80], used the classical Tutte's theorem [Tu47], to show that there are seven locally projective amalgams for $n=2$. Here we use the most difficult and interesting case of Trofimov's theorem [Tr01] to extend the classification to the case $n \geq 3$. We show that besides two infinite series of locally projective amalgams (embedded into the groups $A G L_{n}(2)$ and $O_{2 n}^{+}(2)$ ) there are exactly twelve exceptional ones. Some of the exceptional amalgams are embedded into sporadic simple groups $M_{22}, M_{23}, C_{2}, J_{4}$ and $B M$. For each of the exceptional amalgam $n=3,4$ or 5 .
\end{abstract}

\section{$\S 1$. Locally projective actions}

An amalgam of rank $m$ is a collection

$$
\mathcal{A}=\left\{\left(G^{[i]}, *_{i}\right) \mid 0 \leq i \leq m-1\right\}
$$

of $m$ groups $\left(G^{[i]}, *_{i}\right), 0 \leq i \leq m-1$, such that for all $0 \leq i<j \leq m-1$ the intersection $G^{[i j]}:=G^{[i]} \cap G^{[j]}$ of the element sets is non-empty and the group operations $*_{i}$ and $*_{j}$ coincide, when restricted to $G^{[i j]}$. If $(G, *)$ is a group and $G^{[0]}, G^{[1]}, \ldots, G^{[m-1]}$ are subgroups in $G$, then $\left\{\left(G^{[i]},\left.*\right|_{G^{[i]}}\right) \mid\right.$ $0 \leq i \leq m-1\}$ is an amalgam.

Let $\mathcal{A}=\left\{\left(G^{[i]}, *_{i}\right) \mid 0 \leq i \leq m-1\right\}$ be an amalgam, $(G, *)$ be a group and $\varphi$ be a mapping of the union of the element sets of the groups constituting $\mathcal{A}$ into $G$ such that for every $0 \leq i \leq m-1$ and all $g, h \in G^{[i]}$ the equality

$$
\varphi\left(g *_{i} h\right)=\varphi(g) * \varphi(h)
$$

Received January 6, 2003.

2000 Mathematics Subject Classification: 20B25, 20E08, 20 D08. 
holds (i.e., the restriction of $\varphi$ to each $G^{[i]}$ is a homomorphism). Then the pair $(G, \varphi)$ is called a completion of $\mathcal{A}$ (here $G$ is the completion group and $\varphi$ is the completion map). The completion is said to be faithful if $\varphi$ is injective and generating if $G$ is generated by the image of $\varphi$. A completion $(\widetilde{G}, \widetilde{\varphi})$ is said to be universal if for every completion $(G, \varphi)$ there is a homomorphism $\psi$ of $\widetilde{G}$ into $G$, such that $\varphi$ is the composition of $\widetilde{\varphi}$ and $\psi$. A universal completion is always generating and the universal completion group is unique up to isomorphism. Furthermore, an amalgam possesses a faithful completion (which is not always the case) if and only if its universal completion is faithful.

Whenever the group operations are clear from the context or irrelevant, we simply write

$$
\mathcal{A}=\left\{G^{[i]} \mid 0 \leq i \leq m-1\right\}
$$

for an amalgam of rank $m$ and also for the union of the element sets of the groups constituting the amalgam. We will also drop the explicit reference to the completion maps whenever it does not cause confusion (in this case by 'completion' we mean the completion group). It is known [K60] that for an amalgam $\mathcal{A}=\left\{G^{[0]}, G^{[1]}\right\}$ of rank 2 the universal completion is faithful and the universal completion group is isomorphic to the free product of $G^{[0]}$ and $G^{[1]}$ amalgamated over the common subgroup $G^{[01]}$. The free amalgamated product is infinite whenever $G^{[01]}$ is proper in both $G^{[0]}$ and $G^{[1]}$.

Let $\Gamma$ be a connected undirected regular graph of finite valency $k$ and $G$ be an automorphism group of $\Gamma$ which acts transitively on the set of vertices. We assume that the action of $G$ is locally finite in the sense that for every vertex $x \in \Gamma$ its stabilizer $G(x)$ is a finite subgroup of $G$. Let $\Gamma(x)$ denote the neighbourhood of $x$ in $\Gamma$ (the set of vertices adjacent to $x$ ), so that $|\Gamma(x)|=k$ and for a non-negative integer $i$ put

$$
\Gamma_{i}(x)=\left\{y \mid y \in \Gamma, d_{\Gamma}(x, y)=i\right\}
$$

where $d_{\Gamma}$ is the natural metric on $\Gamma$, so that $\Gamma_{0}(x)=\{x\}$ and $\Gamma_{1}(x)=\Gamma(x)$. An $s$-arc in $\Gamma$ is an ordered sequence $\left(x_{0}, x_{1}, \ldots, x_{s}\right)$ of $(s+1)$ vertices, such that $x_{i+1} \in \Gamma\left(x_{i}\right)$ for $0 \leq i \leq s-1$ and $x_{i+2} \neq x_{i}$ for $0 \leq i \leq s-2$. When a subgroup $H \leq G$ stabilizes a subset $\Xi \subseteq \Gamma$, we write $H^{\Xi}$ for the permutation group on $\Xi$ induced by $H$. For an integer $i$ let $G_{i}(x)$ denote the vertex-wise stabilizer in $G$ of the ball of radius $i$ centred at $x$, so that

$$
G_{i}(x)=\bigcap_{d_{\Gamma}(x, y) \leq i} G(y) .
$$


If $\{x, y\}$ is an edge of $\Gamma$, we put $G(x, y)=G(x) \cap G(y)$ and $G_{i}(x, y)=$ $G_{i}(x) \cap G_{i}(y)$ for $i \geq 1$.

It is well known and easy to prove that a vertex-transitive group $G$ is 2-arc-transitive if and only if the subconstituent $G(x)^{\Gamma(x)}$ is a doubly transitive permutation group. When the action under consideration is $s$ but not $(s+1)$-arc-transitive we say that it is strictly $s$-arc-transitive.

Let $n \geq 2$ be an integer and $q$ be a prime power. We say that a locally finite, vertex-transitive action of $G$ on $\Gamma$ is locally projective of type $(n, q)$ if the subconstituent $G(x)^{\Gamma(x)}$ contains a normal subgroup isomorphic to the special linear group $L_{n}(q)$ acting on the set of 1-dimensional subspaces in its natural module (an $n$-dimensional $G F(q)$-space), so that $k=\left(q^{n}-\right.$ $1) /(q-1)$,

$$
L_{n}(q) \unlhd G(x)^{\Gamma(x)} \leq P \Gamma L(n, q),
$$

and the action is 2-arc-transitive.

The special role of locally projective actions is due to the following result (cf. [W81]).

Proposition 1.1. Let $G$ be a 2-arc-transitive automorphism group of a graph $\Gamma$ and suppose that the subconstituent $G(x)^{\Gamma(x)}$ is one of the known doubly transitive permutation groups. Then either

(i) $G_{1}(x, y)=1$ for every edge $\{x, y\}$ of $\Gamma$, or

(ii) the action of $G$ on $\Gamma$ is locally projective.

From the middle of the 1970s till the beginning of the 1990s the existence problem of an absolute constant $c$ such that $G_{c}(x)=1$ for every locally projective action was open and considered to be extremely difficult. The following result known as Trofimov's theorem was announced in [Tr91a] and proved in the sequence of papers [Tr92], [Tr95a], [Tr95b], [Tr98], [Tr00], [Tr01], [Tr03b].

THEOREM 1.2. For every locally projective action the equality $G_{6}(x)=$ 1 holds.

Let us recall a few standard facts concerning locally projective actions (cf. [W77], [W78], [W82], [Tr91a], [Iv90] or Chapter 9 in [Iv99]).

Let $\Pi_{x}$ denote the projective $G F(q)$-space of rank $n-1$ having $\Gamma(x)$ as the set of points, which is invariant under the action of $G(x)$ (the elements 
of $\Pi_{x}$ are treated as subsets of $\left.\Gamma(x)\right)$. For $y \in \Gamma(x)$ let $\Pi_{x}(y)$ be the residue of $y$ in $\Pi_{x}$ which is the set of subspaces from $\Pi_{x}$ properly containing $y$. Then $\Pi_{x}(y)$ carries the structure of a $G F(q)$-projective space of rank $n-2$.

Proposition 1.3. Suppose that the action of $G$ on $\Gamma$ is locally projective of type $(n, q)$ and let $\{x, y\}$ be an edge of $\Gamma$. Then either

(i) $G$ is 3-arc-transitive and $G(x, y)$ acts flag-transitively on the direct sum of the spaces $\Pi_{x}(y)$ and $\Pi_{y}(x)$, or

(ii) $G$ is strictly 2-arc-transitive and there is a unique isomorphism $\varphi_{x, y}$ of $\Pi_{x}(y)$ onto $\Pi_{y}(x)$ which commutes with the action of $G(x, y)$, furthermore either

(a) $\varphi_{x, y}$ is a collineation, or

(b) $n \geq 4$ and $\varphi_{x, y}$ is a correlation.

Within the proof of Trofimov's theorem, the possible structure of the chief factors of the vertex stabilizer $G(x)$ in a locally projective action was established. This brings one very close to the description of such stabilizers up to isomorphism. The vertex stabilizer itself does not determine the whole group $G$, in any reasonable sense. On the other hand $G$ is a faithful completion of the amalgam

$$
\mathcal{A}=\mathcal{A}(G, \Gamma)=\left\{G^{[0]}=G(x), G^{[1]}=G\{x, y\}\right\}
$$

formed by the vertex and edge stabilizers. The graph $\Gamma$ itself has the (right) cosets of $G^{[0]}$ in $G$ as vertices. Two such cosets $G^{[0]} g$ and $G^{[0]} h$ are adjacent if

$$
G^{[1]} k \cap G^{[0]} g \neq \emptyset \quad \text { and } \quad G^{[1]} k \cap G^{[0]} h \neq \emptyset
$$

for some $k \in G$.

We can treat $\mathcal{A}$ as an abstract amalgam and define $G$ to be the universal completion of $\mathcal{A}$ (which is the free product of $G^{[0]}$ and $G^{[1]}$ amalgamated over the subgroup $\left.G^{[0]} \cap G^{[1]}\right)$, in which case $\Gamma$ is a tree. Thus the description up to isomorphism of the amalgams $\mathcal{A}(G, \Gamma)$ arising from locally projective actions is equivalent to the description of the locally finite automorphism groups of trees inducing locally projective actions.

The full automorphism group of a regular tree of valency at least 3 is 'large' in the sense that it is uncountable and not finitely generated. Every 
locally finite automorphism group we are after is 'small' in the sense that it is countable and finitely generated.

The description of the locally finite 1-arc-transitive automorphism groups of the cubic tree goes back to the classical result of W. Tutte in [Tu47]. There are exactly seven isomorphism types of amalgams $\mathcal{A}=$ $\{G(x), G\{x, y\}\}$ and only five isomorphism types of vertex stabilizers: $Z_{3}$, $S_{3}, S_{3} \times 2, S_{4}$ and $S_{4} \times 2$ (cf. [DM80]).

In the proof of Trofimov's theorem technically the hardest turned out to be the case when $q=2$ and the action is strictly 2-arc-transitive of collineation shape (the case (ii) (a) in Proposition 1.3 for $q=2$ ). The papers [Tr00], [Tr01], [Tr03b] deal almost solely with this case. This is not an incident since the famous examples of locally projective actions related to the flag-transitive Petersen geometries arise in this case. All such geometries have been classified in [Iv99] and [IS02]. There are exactly eight of then and among their automorphism groups we find sporadic simple groups $M_{22}$, $M_{23}, C_{2}, J_{4}, B M$ and certain non-split extensions of these groups by elementary abelian 3-groups.

In the present paper we give the complete classification of the amalgams $\mathcal{A}=\{G(x), G\{x, y\}\}$ coming from locally projective action of type $(n, q)$ where $n \geq 3, q=2$ and the action is strictly 2 -arc-transitive of collineation shape. These amalgams modulo twelve rather remarkable exceptions form two classical infinite series. The precise statement of the main result will be given in the next section after a few further definitions.

\section{§2. The main result}

For the remainder of the paper we assume that the action of $G$ on $\Gamma$ is locally projective of type $(n, q)$, where $q=2$ and $n \geq 3$ (the case $n=2$ is covered by the classical result of W. Tutte on cubic graphs), so that

$$
G(x)^{\Gamma(x)} \cong L_{n}(2) \text { and } \quad k=|\Gamma(x)|=2^{n}-1 .
$$

Furthermore, we assume that the action is strictly 2-arc-transitive and of collineation shape.

Let $\{x, y\}$ be an edge of $\Gamma$. Put $G^{[0]}=G(x), G^{[1]}=G\{x, y\}$ and let

$$
\mathcal{A}=\mathcal{A}(G, \Gamma)=\left\{G^{[0]}, G^{[1]}\right\}
$$

be the amalgam, formed by the vertex and edge stabilizers of the action under consideration. The above conditions on the action of $G$ on $\Gamma$ possess equivalent reformulation in group-theoretical terms: 
(1) $G^{[0]}$ is a finite group, such that $\bar{G}^{[0]}:=G^{[0]} / O_{2}\left(G^{[0]}\right)$ is isomorphic to $L_{n}(2)$;

(2) $G^{[0]} \cap G^{[1]}$ is the full preimage in $G^{[0]}$ of the stabilizer in $\bar{G}^{[0]}$ of a non-zero vector in its natural module $U$ (which is an $n$-dimensional $G F(2)$-space);

(3) $\left[G^{[1]}: G^{[0]} \cap G^{[1]}\right]=2$;

(4) $G^{[1]} / O_{2}\left(G^{[0]} \cap G^{[1]}\right) \cong L_{n-1}(2) \times 2$;

(5) $G^{[1]}$ does not normalize proper subgroups in $G^{[0]} \cap G^{[1]}$ normal in $G^{[0]}$.

The latter condition reflects the fact that the action of $G$ on $\Gamma$ is faithful.

Thus our goal is to describe up to isomorphisms the amalgams $\mathcal{A}=$ $\left\{G^{[0]}, G^{[1]}\right\}$ satisfying the conditions (1) to (5). Within an inductive approach sometimes we will deal with amalgams where (5) fails; therefore it is convenient to have a special name for that condition. If an amalgam $\mathcal{A}$ satisfies (5) it will be called simple.

Consider $\mathcal{A}=\left\{G^{[0]}, G^{[1]}\right\}$ as an abstract amalgam and let $\widetilde{G}$ be the universal completion of $\mathcal{A}$, so that $\widetilde{G}$ is the free product of $G^{[0]}$ and $G^{[1]}$ amalgamated over the common subgroup $G^{[01]}=G^{[0]} \cap G^{[1]}$. A faithful completion $\bar{G}$ of $\mathcal{A}$ can be defined as a homomorphic image of $\widetilde{G}$ such that $\mathcal{A}$ maps bijectively onto its image $\overline{\mathcal{A}}=\left\{\bar{G}^{[0]}, \bar{G}^{[1]}\right\}$ in $\bar{G}$. Having such a faithful completion $\bar{G}$ we can define a graph $\bar{\Gamma}=\Gamma(\mathcal{A}, \bar{G})$ whose vertices are the (right) cosets of $\bar{G}^{[0]}$ in $\bar{G}$ and two such cosets are adjacent if they intersect in a coset of $\bar{G}^{[01]}$. Then the natural action of $\bar{G}$ on $\bar{\Gamma}$ is locally projective and

$$
\mathcal{A}(\bar{G}, \bar{\Gamma}) \cong \mathcal{A}
$$

Furthermore $\Gamma(\mathcal{A}, \bar{G})$ is a tree if and only if $\bar{G}=\widetilde{G}$ and the original graph $\Gamma$ can be recovered as $\Gamma(\mathcal{A}, G)$, since $G$ is clearly a faithful completion of $\mathcal{A}$.

When studying locally projective actions it is convenient to consider the geometric subgraphs in $\Gamma$ defined in the following way (compare Chapter 9 in [Iv99]).

Let $\Pi_{x}$ be the projective geometry on $\Gamma(x)$ invariant under the action of $G(x)$ and let $\varphi_{x, y}$ be the unique collineation of $\Pi_{x}(y)$ onto $\Pi_{y}(x)$, which commutes with the action of $G(x, y)$. Let $2 \leq i \leq n-1$ and $\Delta \subset \Pi_{x}$ be a subspace of (linear) dimension $i$. We identify $\Delta$ with its point-set, which is a $\left(2^{i}-1\right)$-subset of $\Pi_{x}$. A subgraph $\Sigma$ in $\Gamma$ is said to be geometric determined by the subspace $\Delta$ if it is: 
(i) connected;

(ii) contains the vertex $x$;

(iii) $\Sigma \cap \Gamma(x)=\Delta$;

(iv) if $z \in \Sigma$ and $\left(x_{0}=x, x_{1}, \ldots, x_{s}=z\right)$ is an arc joining $x$ with $z$ in $\Sigma$, then

$$
\Sigma \cap \Gamma\left(x_{s}\right)=\varphi_{x_{s-1}, x_{s}}\left(\Sigma \cap \Gamma\left(x_{s-1}\right)\right)
$$

It is easy to see that if $\Sigma$ exists then it is unique and its valency is $2^{i}-1$ (we will denote this subgraph by $\Gamma_{\Delta}$ ). The subgraph $\Gamma_{\Delta}$ always exists if $\Gamma$ is a tree.

The geometric subgraph $\Gamma_{\Delta}$ can also be defined in terms of a subgroup in $G$ (below we assume that $y \in \Delta$ ). Indeed, let $H^{[0]}$ be the stabilizer in $G(x)=G^{[0]}$ of the subspace $\Delta$ and $H^{[1]}$ be the stabilizer in $G^{[1]}=G\{x, y\}$ of the set

$$
\Delta \cup \varphi_{x, y}(\Delta)
$$

in which case $H^{[1]}=\left\langle H^{[0]} \cap G(x, y), \tau\right\rangle$, where

$$
\tau \in(G\{x, y\} \backslash G(x, y)) \cap O_{2}(G(\{x, y\}))
$$

so that $\tau$ induces the collineation $\varphi_{x, y}$. Put

$$
H:=\left\langle H^{[0]}, H^{[1]}\right\rangle
$$

The following result was established in [Iv90] (cf. Lemma 9.6.4 in [Iv99]) and we sketch its proof here.

LEMma 2.1. The geometric subgraph $\Gamma_{\Delta}$ exists if and only if $H$ is a proper subgroup of $G$. If $\Gamma_{\Delta}$ exists then $H$ is the full stabilizer of $\Gamma_{\Delta}$ in $G$ and the action of $H$ on $\Gamma_{\Delta}$ is locally projective of type $(i, 2)$.

Proof. If $\Gamma_{\Delta}$ exists then it is by the uniqueness property that $H$ is its stabilizer in $G$, therefore $H$ is a proper subgroup of $G$ in this case. Suppose that $H$ is proper in $G$. Define $\Gamma_{\Delta}$ to be the subgraph induced by the images of $x$ under $H$. Since $H^{[0]}$ is maximal in $G^{[0]}$ and $H \not \leq G^{[0]}$ we have $G^{[0]} \cap H=H^{[0]}$ and similarly $G^{[1]} \cap H=H^{[1]}$. Therefore $\Delta$ is the set of neighbours of $x$ in $\Gamma_{\Delta}$. Since $H$ acts transitively on the vertex-set of $\Gamma_{\Delta}$ it is clear that $\Gamma_{\Delta}$ satisfies all the imposed conditions. 
If $\Gamma$ is a tree and $G$ is the free amalgamated product of $G^{[0]}$ and $G^{[1]}$, then $H$ is always proper isomorphic to the free amalgamated product of $H^{[0]}$ and $H^{[1]}$ over the subgroup $H^{[0]} \cap H^{[1]}$ and $\Gamma_{\Delta}$ is a subtree in $\Gamma$.

Let $K$ denote the kernel of the action of $H$ on $\Gamma_{\Delta}$. Then $K$ is the largest subgroup in $H^{[0]} \cap H^{[1]}$, normal in both $H^{[0]}$ and $H^{[1]}$. Therefore

$$
\mathcal{A}\left(H / K, \Gamma_{\Delta}\right)=\left\{H^{[0]} / K, H^{[1]} / K\right\}
$$

is the amalgam formed by the vertex and edge stabilizers in the faithful locally projective action of type $(i, 2)$ which enables us to follow an inductive approach. Since $K$ is contained in $G^{[0]}$ it can not be normal in $G$ unless it is trivial and by Lemma 2.1 this gives the following.

LemmA 2.2. If $K \neq 1$ then $H$ is proper in $G$ and $\Gamma_{\Delta}$ exists.

Certainly the structure of $H$ depends on the particular choice of $G$. On the other hand, the isomorphism types of the kernel $K$ and the amalgam $\mathcal{A}\left(H / K, \Gamma_{\Delta}\right)$ are internal characteristics of the amalgam $\mathcal{A}(G, \Gamma)$. Another important characteristic is the group

$$
\widehat{H}:=H /\left(K C_{H}(K)\right),
$$

isomorphic to the image of $H$ in the group of outer automorphisms of $K$.

We refer to [P94] for basic definitions concerning diagram geometries. Suppose that $\Gamma$ (with respect to the action of $G$ ) contains a complete family of geometric subgraphs (i.e., $\Gamma_{\Delta}$ exists for every vertex $x$ and every $i$ dimensional subspace $\Delta$ in $\Pi_{x}$ for $\left.2 \leq i \leq n-1\right)$. Define a geometry $\mathcal{G}=\mathcal{G}(G, \Gamma)$ of rank $n$ over the set of types $\{0,1, \ldots, n-1\}$ in which the elements of types zero and one are, respectively the vertices and edges and for $2 \leq i \leq n-1$ the elements of type $i$ are the geometric subgraphs $\Gamma_{\Delta}$ where $\Delta$ is an $i$-dimensional subspace in $\Pi_{x}$ for some vertex $x$. The incidence relation is via inclusion. Then $\mathcal{G}$ belongs to a diagram of the shape

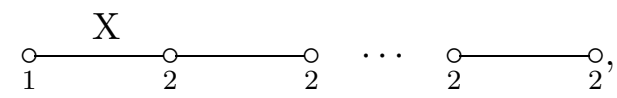

where $\underset{1}{\circ} \quad \mathrm{X} \quad{ }_{2}^{\circ}$ is the geometry of vertices and edges of a geometric subgraph of valency 3 (the types on the diagram increase rightwards). Notice that for a vertex $x \in \Gamma$ its residue $\operatorname{res}_{\mathcal{G}}(x)$ in $\mathcal{G}$ coincides with $\Pi_{x}$. The action of $G$ on $\mathcal{G}$ is flag-transitive. 
Let $\{y\} \subset \Delta^{[2]} \subset \cdots \subset \Delta^{[n-1]}$ be a maximal flag in $\Pi_{x}, \Sigma^{[i]}=\Gamma_{\Delta^{[i]}}$ and $G^{[i]}$ be the stabilizer of $\Sigma^{[i]}$ in $G$ for $2 \leq i \leq n-1$. Then

$$
\{x\} \subset\{x, y\} \subset \Sigma^{[2]} \subset \cdots \subset \Sigma^{[n-1]}
$$

is a maximal flag in $\mathcal{G}$ and

$$
\left\{G^{[0]}=G(x), G^{[1]}=G\{x, y\}, G^{[2]}, \ldots, G^{[n-1]}\right\}
$$

is the corresponding amalgam of maximal parabolics.

For an element $\alpha \in \mathcal{G}$ let $\operatorname{res}_{\mathcal{G}}^{+}(\alpha)$ and $\operatorname{res}_{\mathcal{G}}^{-}(\alpha)$ denote the set of elements incident to $\alpha$ whose type is greater than the type of $\alpha$ and less than the type of $\alpha$, respectively. Let $G_{+}^{[i]}, G_{-}^{[i]}$ and $G_{0}^{[i]}$ denote the element-wise stabilizers in $G^{[i]}$ of $\operatorname{res}_{\mathcal{G}}^{+}\left(\Sigma^{[i]}\right), \operatorname{res}_{\mathcal{G}}^{-}\left(\Sigma^{[i]}\right)$ and $\operatorname{res}_{\mathcal{G}}\left(\Sigma^{[i]}\right)$, respectively. Then $G_{-}^{[i]}$ is the kernel of the action of $G^{[i]}$ on $\Sigma^{[i]}$ and it is the largest subgroup in $G(x, y) \cap G^{[i]}$ normal in both $G(x) \cap G^{[i]}$ and $G\{x, y\} \cap G^{[i]}$.

As above put

$$
\widehat{G}^{[i]}=G^{[i]} /\left(G_{-}^{[i]} C_{G^{[i]}}\left(G_{-}^{[i]}\right)\right)
$$

and say that $G$ is constrained at level $i$ if $C_{G^{[i]}}\left(G_{-}^{[i]}\right) \leq G_{-}^{[i]}$.

Let $\mathcal{G}$ be an arbitrary geometry of rank $n$ belonging to the above diagram and $G$ be a flag-transitive automorphism group of $\mathcal{G}$ such that the stabilizer in $G$ of a zero-type element induces the full automorphism group $L_{n}(2)$ of the corresponding residue. Let $\Gamma$ be the derived graph of $\mathcal{G}$ which is a graph on the set of elements of type zero in $\mathcal{G}$ in which two such elements are adjacent if in $\mathcal{G}$ they are incident to a common element of type one. Then the action of $G$ on $\Gamma$ is locally projective of type $(n, 2)$. This observation enables us to switch from graph to geometry and back.

Now before formulating the main results it only remains to recall some known examples.

ExAmple 1. Let $V_{n}(2)$ be an $n$-dimensional $G F(2)$-space and $G=$ $A G L_{n}(2)$ be the group of affine transformations of $V_{n}(2)$. Let $\Gamma \cong K_{2^{n}}$ be the complete graph on the set of vectors in $V_{n}(2)$. Then the natural action of $G$ on $\Gamma$ is locally projective of type $(n, 2), G_{1}(x)=1, \Gamma$ contains a complete family of geometric subgraphs which are cosets of the proper 
subspaces in $V_{n}(2), \widehat{G}^{[i]} \cong L_{i}(2), G$ is not constrained, the geometry $\mathcal{G}(G, \Gamma)$ is the affine space with the diagram

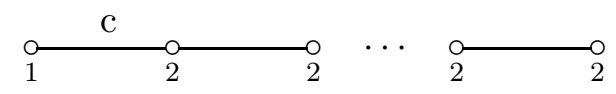

and

$$
\mathcal{A}\left(A G L_{n}(2), K_{2^{n}}\right)=\left\{L_{n}(2), 2^{n-1}: L_{n-1}(2) \times 2\right\} .
$$

ExAmPLE 2. Let $V_{2 n}(2)$ be a $2 n$-dimensional $G F(2)$-space equipped with a non-degenerate quadratic form $f$ of plus type, $n \geq 3$. Let $G=O_{2 n}^{+}(2)$ be the corresponding orthogonal group (which contains the simple group $\Omega_{2 n}^{+}(2)$ with index 2$)$. Let $\Gamma=D^{+}(2 n, 2)$ be the dual polar graph which is the graph on the set of maximal ( $n$-dimensional) totally singular subspaces in $V_{2 n}(2)$ with respect to the form $f$, where two subspaces are adjacent if their intersection has dimension $n-1$. Then (cf. [CP82]) the action of $G$ on $\Gamma$ is locally projective of type $(n, 2), G_{2}(x)=1, G_{1}(x)$ is the exterior square of $x$ (when the latter is considered as a natural module for $G(x)^{\Gamma(x)} \cong L_{n}(2)$ ). $\Gamma$ contains a complete family of geometric subgraphs, which are indexed by the subspaces in $V_{2 n}(2)$ totally singular with respect to $f, \widehat{G}^{[i]} \cong O_{2 i}^{+}(2)$, the group $G$ is constrained, the geometry $\mathcal{G}(G, \Gamma)$ is the building with the diagram

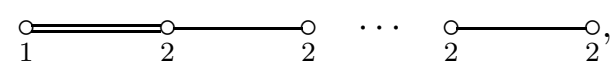

a geometric subgraph of valency 3 is the complete bipartite graph $K_{3,3}$ with the automorphism group $S_{3} \prec S_{2} \cong O_{4}^{+}(2)$.

Example 3 . Let $\mathcal{G}$ be a $P$-geometry (a Petersen type geometry [Iv99]) of rank $n \geq 3$ with the diagram

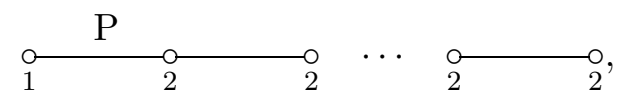

where $\underset{1}{\circ} \quad \mathrm{P} \__{2}$ is the geometry of vertices and edges of the Petersen graph, whose automorphism group is $S_{5} \cong O_{4}^{-}(2)$. If $G$ is a flag-transitive automorphism group of $\mathcal{G}$ then the action of $G$ on the derived graph of $\mathcal{G}$ is locally projective of type $(n, 2)$. It is known [Iv99], [IS02] that there are exactly eight flag-transitive $P$-geometries:

$$
\begin{aligned}
& \mathcal{G}\left(M_{22}\right), \quad \mathcal{G}\left(3 \cdot M_{22}\right), \quad \mathcal{G}\left(M_{23}\right), \quad \mathcal{G}\left(\mathrm{Co}_{2}\right), \\
& \mathcal{G}\left(3^{23} \cdot C_{2}\right), \quad \mathcal{G}\left(J_{4}\right), \quad \mathcal{G}(B M), \quad \mathcal{G}\left(3^{4371} \cdot B M\right) \text {. }
\end{aligned}
$$

Every flag-transitive automorphism group of $\mathcal{G}$ is constrained at level 2 . 
EXAMPLE 4. Let $V=V_{4}(2)$ be a 4-dimensional $G F(2)$-space, $L=$ $G L(V) \cong L_{4}(2)$ and $\delta$ be an outer automorphism of $L$ of order 2 (so that $\left.\langle L, \delta\rangle \cong S_{8} \cong O_{6}^{+}(2)\right)$. Let $H=\{(A, B) \mid A, B \in L\}$ be the direct product of two copies of $L$. Let $\sigma_{1}$ and $\sigma_{2}$ be automorphisms of $H$ such that

$$
\begin{aligned}
& \sigma_{1}:(A, B) \longmapsto(B, A) \text { and } \\
& \sigma_{2}:(A, B) \longmapsto\left(A^{\delta}, B^{\delta}\right) .
\end{aligned}
$$

Notice that $\sigma_{1}$ and $\sigma_{2}$ commute. Let $G$ be the semidirect product (with respect to the natural action) of $H$ and $\left\langle\sigma_{1}, \sigma_{2}\right\rangle \cong 2^{2}$. Let $\Delta$ be the conjugacy class of $\sigma_{1}$ in $G$. It is easy to see that $\Delta=\{\sigma(l) \mid l \in L\}$ and

$$
C_{H}(\sigma(l))=\left\{\left(A, A^{l}\right) \mid A \in L\right\}
$$

(isomorphic to $L$ ).

Let $P_{1}$ be the stabilizer of a non-zero vector $u \in U$, so that $P_{1} \cong$ $2^{3}: L_{3}(2)$ and the non-identity elements in $O_{2}\left(P_{1}\right)$ are the transvections having $u$ as the centre. Let $X$ be the subgroup in $G$ generated by $\sigma_{1}$ and $\left\{(A, A) \mid A \in P_{1}\right\} \leq C_{H}\left(\sigma_{1}\right)$. Then

$$
X \cong P_{1} \times\left\langle\sigma_{1}\right\rangle \cong 2^{3}: L_{3}(2) \times 2
$$

and $X$ is self-normalized in $G$. Let $l$ be a transvection from $O_{2}\left(P_{1}\right)$ and let $W$ be the axis of $l$. Then $W$ is a 3 -subspace in $U$ containing $u$ and if $P_{3} \cong 2^{3}: L_{3}(2)$ is the stabilizer of $W$ in $L$, then

$$
P_{1} \cap P_{3}=C_{L}(l) \cong 2_{+}^{1+4} \cdot S_{3} .
$$

Let $Y$ be the subgroup in $G$, generated by $\sigma(l)$ and $\left\{\left(A, A^{l}\right) \mid A \in P_{2}\right\} \leq$ $C_{H}(\sigma(l))$. Then $X$ and $Y$ are conjugate in $G$ and the intersection $X \cap Y$ is generated by $\sigma_{1}, \sigma(l)$ and

$$
\left\{(A, A) \mid A \in P_{1} \cap P_{3}=C_{L}(l)\right\} .
$$

Furthermore, the intersection has index 7 in both $X$ and $Y$.

Let $\Gamma$ be the graph whose vertices are the $G$-conjugates of $X$ and whose edges are the images of $\{X, Y\}$ under $G$ (acting by conjugation). Then $\Gamma$ is connected, $G$ induces on $\Gamma$ a locally projective action of type $(3,2)$ with $G_{2}(x) \cong 2, G_{1}(x) / G_{2}(x) \cong 2^{3}$.

A geometric subgraph $\Sigma^{[2]}$ of valency 3 in $\Gamma$ containing $X$ and $Y$ can be obtained as follows. Let $U$ be a 2 -space containing $u$ and contained in $W$ and $P_{2}$ be the stabilizer of $U$ in Aut $L=\langle L, \delta\rangle$. Then

$$
P_{2} \cong 2^{4}: O_{4}^{+}(2)
$$


is the semidirect product of $Q_{2}:=\mathrm{O}_{2}\left(P_{2}\right)$ which is elementary abelian of order $2^{4}$ and $O_{4}^{+}(2) \cong S_{3}<S_{2}$, so that $Q_{2}$ is the natural module for the latter.

Then $\Sigma^{[2]}$ is formed by the subgroups (conjugates of $X$ in $G$ ), each being generated by $\sigma(l)$ for some $l \in Q_{2}$ and $\{(A, A) \mid A \in R\} \leq C_{H}(\sigma(l))$ for the stabiliser $R$ of a 1 -subspace contained in $U$ or of a 3 -subspace containing $U$ (therefore $\Sigma^{[2]}$ contains exactly $16 \times 6=96$ vertices). It is easy to see that the vertex-wise stabilizer $G_{-}^{[2]}$ of $\Sigma^{[2]}$ is elementary abelian of order $2^{5}$ generated by $\sigma_{1}$ and $\left\{(A, A) \mid A \in Q_{2}\right\}$. This subgroup is selfcentralized in $G$ and

$$
\widehat{G}^{[2]} \cong P_{2} \cong 2^{4}: O_{4}^{+}(2)
$$

in particular $G$ is a completion of $\{G(x), G\{x, y\}\}$ which is constrained at level 2 (this example first appeared on pp. 191-192 in [Tr00].)

TheOREm 1. Let $\Gamma$ be a graph and $G$ be a group of locally finite automorphisms of $\Gamma$ whose action is locally projective of type $(n, 2)$ for $n \geq 3$. Suppose further that the action is strictly 2-arc-transitive of collineation shape. Let $\{x, y\}$ be an edge of $\Gamma$ and $\mathcal{A}=\mathcal{A}(G, \Gamma)=\{G(x), G\{x, y\}\}$ be the amalgam formed by the vertex and edge stabilizers. Then exactly one of the following holds:

(i) $\mathcal{A} \cong \mathcal{A}\left(A G L_{n}(2), K_{2^{n}}\right)$;

(ii) $\mathcal{A} \cong \mathcal{A}\left(O_{2 n}^{+}(2), D^{+}(2 n, 2)\right)$;

(iii) $\mathcal{A}$ is isomorphic to one of the twelve exceptional amalgams in Table 1.

If $\mathcal{A}$ is one of the amalgams $\mathcal{A}_{2}^{(2)}, \mathcal{A}_{3}^{(5)}, \mathcal{A}_{4}^{(1)}, \mathcal{A}_{4}^{(3)}, \mathcal{A}_{4}^{(4)}$ and $\mathcal{A}_{5}^{(2)}$ and $G$ is the group given in the last column of the row corresponding to $\mathcal{A}$, then $\mathcal{A}$ is formed by the stabilizers in $G$ of a vertex and an edge in the derived graph of the Petersen geometry $\mathcal{G}(G)$. The amalgam $\mathcal{A}_{3}^{(4)}$ is from Example 4 . The amalgam $\mathcal{A}_{5}^{(1)}$ corresponds to the locally projective action of the fourth Janko group $J_{4}$ on a graph of valency 31 (cf. [Iv87] and [SW88]). The remaining amalgams will be discussed within the proof of Theorem 1 .

Corollary 1. Let $\mathcal{A}$ be an amalgam in Theorem 1 for some $n \geq 3$. Let $G$ be a faithful completion of $\mathcal{A}$ and $2 \leq i \leq n-1$. Then one of the following holds:

(i) $G_{-}^{[i]}$ is non-trivial; 
Table 1: Exceptional Amalgams

\begin{tabular}{|c|c|c|c|c|c|c|}
\hline$n$ & $\mathcal{A}$ & $\frac{G^{[0]} / O_{2}\left(G^{[0]}\right)}{O_{2}\left(G^{[0]}\right)}$ & $\frac{\widehat{G}^{[2]}}{K^{[2]}}$ & $\frac{\widehat{G}^{[3]}}{K^{[3]}}$ & $\frac{\widehat{G}^{[4]}}{K^{[4]}}$ & $\begin{array}{c}\text { completions } \\
\text { constrained } \\
\text { at level } 2\end{array}$ \\
\hline \multirow[t]{5}{*}{3} & $\mathcal{A}_{3}^{(1)}$ & $\frac{L_{3}(2)}{2^{3}}$ & $\frac{S_{3} 2 S_{2}}{2^{4}}$ & & & - \\
\hline & $\mathcal{A}_{3}^{(2)}$ & $\frac{L_{3}(2)}{2^{3}}$ & $\frac{S_{5}}{2^{4}}$ & & & $M_{22}$ \\
\hline & $\mathcal{A}_{3}^{(3)}$ & $\frac{L_{3}(2)}{2^{3}}$ & $\frac{S_{5}}{2^{4}}$ & & & - \\
\hline & $\mathcal{A}_{3}^{(4)}$ & $\frac{L_{3}(2)}{2^{3} \times 2}$ & $\frac{2^{4}:\left(S_{3} 2 S_{2}\right)}{2^{5}}$ & & & $\left(S_{8} \backslash 2\right)^{+}$ \\
\hline & $\mathcal{A}_{3}^{(5)}$ & $\frac{L_{3}(2)}{2^{3} \times 2}$ & $\frac{S_{5}}{2^{5}}$ & & & Aut $M_{22}$ \\
\hline \multirow[t]{5}{*}{4} & $\mathcal{A}_{4}^{(1)}$ & $\frac{L_{4}(2)}{1}$ & $\frac{S_{5}}{2^{4}: 3}$ & $\frac{1}{1}$ & & $M_{23}$ \\
\hline & $\mathcal{A}_{4}^{(2)}$ & $\frac{L_{4}(2)}{2^{6}}$ & $\frac{S_{5} \times 2}{2_{+}^{1+8}: S_{3}}$ & $\frac{L_{6}(2)}{2^{6}}$ & & $A_{64}$ \\
\hline & $\mathcal{A}_{4}^{(3)}$ & $\frac{L_{4}(2)}{2^{1+4+6}}$ & $\frac{S_{5}}{2^{4+10} \cdot S_{3}}$ & $\frac{\text { Aut } M_{22}}{2^{10}}$ & & $\mathrm{Co}_{2}$ \\
\hline & $\mathcal{A}_{4}^{(4)}$ & $\frac{L_{4}(2)}{2^{4+4+6}}$ & $\frac{S_{5}}{2^{3+12+2} \cdot S_{3}}$ & $\frac{3 \cdot \text { Aut } M_{22}}{2_{+}^{1+12}}$ & & $J_{4}$ \\
\hline & $\mathcal{A}_{4}^{(5)}$ & $\frac{L_{4}(2)}{2^{4+4+6}}$ & $\frac{S_{5} \times 2}{2^{3+12+2} \cdot S_{3}}$ & $\frac{L_{6}(2): 2}{2_{+}^{1+12}}$ & & $A_{256}$ \\
\hline \multirow[t]{2}{*}{5} & $\mathcal{A}_{5}^{(1)}$ & $\frac{L_{5}(2)}{2^{10}}$ & $\frac{S_{5}}{2^{3+12} \cdot L_{3}(2)}$ & $\frac{\text { Aut } M_{22}}{2_{+}^{1+12}: 3}$ & $\frac{1}{1}$ & $J_{4}$ \\
\hline & $\mathcal{A}_{5}^{(2)}$ & $\frac{L_{5}(2)}{2^{5+5+10+10}}$ & $\frac{S_{5}}{2^{3} \cdot\left[2^{32}\right] \cdot L_{3}(2)}$ & $\frac{\text { Aut } M_{22}}{2^{2+10+20} \cdot S_{3}}$ & $\frac{\mathrm{Co}_{2}}{2_{+}^{1+22}}$ & $B M$ \\
\hline
\end{tabular}


(ii) $\mathcal{A} \cong \mathcal{A}_{4}^{(1)}$ and $i=3$;

(iii) $\mathcal{A} \cong \mathcal{A}_{5}^{(1)}$ and $i=4$.

Furthermore, if $\mathcal{A}$ is neither $\mathcal{A}_{4}^{(1)}$ nor $\mathcal{A}_{5}^{(1)}$, then $\Gamma(\mathcal{A}, G)$ contains a complete family of geometric subgraphs.

Proof. Consider $G=A G L_{n}(2)$ as a completion of $\mathcal{A}\left(A G L_{n}(2), K_{2^{n}}\right)$. Then $G_{-}^{[i]}$ is the vector-wise stabilizer in $G$ of an $i$-dimensional subspace in the $n$-dimensional affine space. It is easy to check that this subgroup is non-trivial for $2 \leq i \leq n-1$. Similarly, if $G=O_{2 n}^{+}(2)$ is considered as a completion of $\mathcal{A}\left(O_{2 n}^{+}(2), D(2 n, 2)\right)$ then $G_{-}^{[i]}$ is the elementwise stabilizer of the set of maximal totally singular subspace in the $2 n$-dimensional orthogonal space that contain a given totally singular $2(n-i)$-dimensional subspace. This subgroup is also known to be non-trivial (cf. [Iv04] for details). For the exceptional cases the result is immediate from Table 1 . The last sentence of the assertion is by Lemma 2.2 .

For both $\mathcal{A}_{4}^{(1)}$ and $\mathcal{A}_{5}^{(1)}$ we know completions where some geometric subgraphs are missing. If $\mathcal{A} \cong \mathcal{A}_{5}^{(1)}$ and $G \cong J_{4}$ then $\Sigma^{[4]}$ does not exist. In this case the subamalgam

$$
\left\{G^{[4]} \cap G(x), G^{[4]} \cap G\{x, y\}\right\}
$$

is isomorphic to $\mathcal{A}_{4}^{(4)}$ and it generates the whole $J_{4}$. A completion of $\mathcal{A}_{4}^{(1)}$ where $\Sigma^{[3]}$ does not exist was constructed in [IP04] (the completion group is isomorphic to $\left.S L_{20}(13)\right)$.

By Corollary 1 if the amalgams $\mathcal{A}_{4}^{(1)}$ and $\mathcal{A}_{5}^{(1)}$ are excluded then there is a complete family of geometric subgraphs and hence $G$ acts flag-transitively on a geometry with diagram of the form

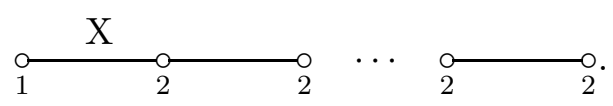

In fact there are some restrictions on the leftmost edge in the diagram (which symbolises the geometry of vertices and edges of $\Sigma^{[2]}$ ). Indeed, let $\Xi$ be the cubic graph associated with the embedding into $\widehat{G}^{[2]}$ of the images in $\widehat{G}^{[2]}$ of $G^{[2]} \cap G^{[0]}$ and $G^{[2]} \cap G^{[1]}$. Then $\Sigma^{[2]}$ possesses a local isomorphism onto $\Xi$ which commutes with the action of $G^{[2]}$. On the other hand in all cases in Table 1 the group $G^{[2]}$ possesses a homomorphism either onto $O_{4}^{+}(2) \cong S_{3} 2 S_{2}$ or onto $O_{4}^{-}(2) \cong S_{5}$. This observation incorporated with the well known properties of the classical amalgams give the following 
COROllary 2. Let $\mathcal{G}$ be a geometry of rank $n \geq 3$ over the set $\{0,1$, $\ldots, n-1\}$ of types. Suppose that $\mathcal{G}$ belongs to a diagram of the form

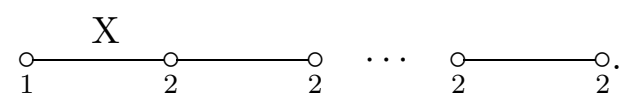

Let $G$ be a flag-transitive automorphism group of $\mathcal{G}$ such that the stabilizer of an element of type zero induces $L_{n}(2)$ on the corresponding residue. Let $\Phi$ be a flag of type $\{2,3, \ldots, n-1\}, \mathcal{F}$ be the residue of $\Phi$ in $\mathcal{G}$ (with the diagram $\mathrm{X}_{1} \mathrm{X}_{2}$ ) and $F$ be the action induced on $\mathcal{F}$ by the stabilizer of $\Phi$ in $G$. Then $\mathcal{F}$ possesses a morphism $\chi$ commuting with $F$ onto one of the following three geometries:

(a) the affine plane $\underset{1}{\stackrel{\mathrm{c}}{\mathrm{O}}}{ }_{2}^{\circ}$ of order 2 (the complete graph $K_{4}$ ) with the automorphism group $S_{4}$;

(b) the generalized digon $\underset{1}{\stackrel{2}{2}}$ (the complete bipartite graph $K_{3,3}$ ) with the automorphism group $S_{3}$ 乙 $S_{2} \cong O_{4}^{+}(2)$;

(c) the Petersen graph $\underset{1}{\circ} \quad \mathrm{P}_{2}^{\circ}$ with the automorphism group $S_{5} \cong$ $\mathrm{O}_{4}^{-}(2)$.

Remark. There is another instance when the rank 2 geometries in (a) to (c) above appear together. Let $\mathcal{Q}$ be the generalized quadrangle of order $(2,2)$ with the automorphism group $S p_{4}(2) \cong S_{6}$. Then $\mathcal{Q}$ possesses exactly three classes of geometric hyperplanes with representatives $\mathcal{H}_{0}, \mathcal{H}_{+}$and $\mathcal{H}_{-}$ and stabilizers $2 \times S_{4}, O_{4}^{+}(2)$ and $O_{4}^{-}(2)$, respectively. The complement of $\mathcal{H}_{0}$ has the geometry ${\underset{1}{\mathrm{O}}}_{\mathrm{C}} \mathrm{C}_{2}$ as a quotient, while the complements to $\mathcal{H}_{+}$ and $\mathcal{H}_{-}$are the geometries $\underset{1}{\stackrel{2}{\rightleftharpoons}}$ and $\underset{1}{\stackrel{\mathrm{P}}{\mathrm{P}_{2}}}$, respectively.

It must be clear that Corollary 2 follows already from the classification of the amalgams for $n=3$.

The following proposition is a summary of the known characterisations of the completions of the amalgams in Theorem 1 that are constrained at level 2 (more specifically when the morphism $\chi$ in Corollary 2 is an isomorphism).

Proposition 2.3. Let $\mathcal{A}$ be one of the amalgams in Theorem 1 and $G$ be the universal completion of $\mathcal{A}$. Let $M$ be a complement to $Z\left(G_{-}^{[2]}\right)$ in 
$C_{G^{[2]}}\left(G_{-}^{[2]}\right)$ (if such a complement exists at all), let $N$ be the normal closure of $M$ in $G$. Let $\bar{G}$ be a faithful completion of $\mathcal{A}$ such that the kernel $K$ of the natural homomorphism of $G$ onto $\bar{G}$ contains $N$. Then

(i) if $\mathcal{A} \cong \mathcal{A}_{3}^{(1)}$ or $\mathcal{A}_{3}^{(3)}$ then $\bar{G}$ does not exist;

(ii) if $\mathcal{A} \cong \mathcal{A}\left(O_{2 n}^{+}(2), D^{+}(2 n, 2)\right)$ then $K=N$ and $\bar{G} \cong O_{2 n}^{+}(2)$;

(iii) if $\mathcal{A} \cong \mathcal{A}_{3}^{(2)}$ then either $K=N$ and $\bar{G} \cong 3 \cdot M_{22}$ or $[K: N]=3$ and $\bar{G} \cong M_{22}$

(iv) if $\mathcal{A} \cong \mathcal{A}_{3}^{(5)}$ then either $K=N$ and $\bar{G} \cong 3 \cdot$ Aut $M_{22}$ or $[K: N]=3$ and $\bar{G} \cong$ Aut $M_{22}$;

(v) if $\mathcal{A} \cong \mathcal{A}_{4}^{(1)}$ then $K=N$ and $\bar{G} \cong M_{23}$;

(vi) if $\mathcal{A} \cong \mathcal{A}_{4}^{(3)}$ then either $K=N$ and $\bar{G} \cong 3^{23} \cdot C o_{2}$ or $[K: N]=3^{23}$ and $\bar{G} \cong \mathrm{Co}_{2}$;

(vii) if $\mathcal{A} \cong \mathcal{A}_{4}^{(4)}$ or $\mathcal{A}_{5}^{(1)}$ then $K=N$ and $\bar{G} \cong J_{4}$;

(viii) if $\mathcal{A} \cong \mathcal{A}_{5}^{(2)}$ then either $K=N$ and $\bar{G} \cong 3^{4371} \cdot B M$, or $[K: N]=3^{4371}$ and $\bar{G} \cong B M$.

Proof. Put $\bar{\Gamma}=\Gamma(\mathcal{A}, \bar{G})$. Then the geometric subgraph $\bar{\Sigma}^{[2]}$ in $\bar{\Gamma}$ is either the complete bipartite graph $K_{3,3}$ or the Petersen graph, in particular the girth (the length of the shortest cycle) of $\Gamma$ is 3,4 or 5 . If the girth of $\bar{\Gamma}$ would be 3 it would be the complete graph, which is clearly impossible. In the girth 4 case we apply the main result of [CP82] which gives (ii) and excludes the case of $\mathcal{A}_{3}^{(1)}$-amalgam. In [Iv90] (cf. Proposition 9.9.2 and Section 9.11 in [Iv99]) the girth 5 case was reduced to the flag-transitive $P$-geometries classified in [Iv99] and [IS02]. Notice that at this stage we are not using any information on the amalgams $\mathcal{A}_{3}^{(1)}$ and $\mathcal{A}_{3}^{(3)}$ besides those given in Table 1 . The point is that the only group acting locally projectively on $D^{+}(6,2)$ is $O_{6}^{+}(2) \cong S_{8}$ and the only group acting locally projectively on the derived graph of the $P$-geometry of $3 \cdot M_{22}$ with vertex stabilizer isomorphic to $2^{3}: L_{3}(2)$ is $3 \cdot M_{22}$ itself. Thus there is no space for extra amalgams.

In Section 10 we will use the following characterization of $\mathrm{Co}_{2}$ as a completion of $\mathcal{A}_{4}^{(3)}$.

Proposition 2.4. Let $G$ be a faithful completion of the amalgam $\mathcal{A}_{4}^{(3)}$ that is constrained at level 3 ( so that $G^{[3]} \cong 2^{10}$. Aut $M_{22}$ ). Then $G \cong \mathrm{Co}_{2}$. 
Proof. If $\Gamma=\Gamma\left(\mathcal{A}_{4}^{(3)}, G\right)$, then by our hypothesis the action $G^{[3]} / G_{-}^{[3]}$ of $G^{[3]}$ on $\Sigma^{[3]}$ is isomorphic to Aut $M_{22}$ and therefore $\Sigma^{[3]}$ is isomorphic to the derived graph of the $P$-geometry $\mathcal{G}\left(M_{22}\right)$. Hence $\Sigma^{[2]}$ is the Petersen graph and by Proposition 2.3 (vi) $G$ is either $3^{23} \cdot \mathrm{Co}_{2}$ or $\mathrm{Co}_{2}$. Finally $G \cong 3^{23} \cdot \mathrm{Co}_{2}$ is not constrained at level 3 , since $G^{[3]} \cong\left(2^{10} \times 3\right) \cdot$ Aut $M_{22}$ in that case, hence the result.

Our terminology and notation are mostly standard if $A, B$ and $C$ are groups and $\varphi: A \rightarrow C, \psi: B \rightarrow C$ are surjective homomorphisms then the sub-direct product of $A$ and $B$ with respect to $\varphi$ and $\psi$ (also known as the pull-back) is defined as the subgroup in the direct product $A \times B=\{(a, b) \mid$ $a \in A, b \in B\}$ consisting of the pairs $(a, b)$ such that $\varphi(a)=\psi(b)$. Usually the homomorphisms $\varphi$ and $\psi$ are determined by $C$ and we talk about the sub-direct product of $A$ and $B$ with respect to $C$.

\section{§3. Some cohomology of $L_{n}(2)$}

Let $L \cong L_{n}(2)$ for $n \geq 3$ and $U$ be a natural module of $L$, i.e., an $n$-dimensional $G F(2)$-space on which $L$ acts in the natural way. In the case when $L=G(x) / G_{1}(x)$, in order to distinguish $U$ from its dual we assume that there is a bijection $\iota$ of $\Gamma(x)$ onto the set of non-zero vectors in $U$ which commutes with the action of $L$. Let $U^{[i]}$ denote the $i$-exterior power of $U$, so that $U=U^{[1]}, \operatorname{dim} U^{[i]}=\left(\begin{array}{c}n \\ i\end{array}\right)$ for $0 \leq i \leq n$ and $U^{[n-1]}$ is the dual of $U$ which will also be denoted by $U^{*}$.

Sometimes we will identify $\Gamma(x)$ and $U^{\#}$ through the bijection $\iota$, so that $y \in \Gamma(x)$ is considered as a vector in the natural module $U$. If $1 \leq i \leq n$ then by $\left[\begin{array}{c}U \\ i\end{array}\right]$ we denote the set of all $i$-dimensional subspaces in $U$. It is well-known (cf. Lemma 3.1.3 in [IS02]) that there is an injection

$$
d_{i}:\left[\begin{array}{c}
U \\
i
\end{array}\right] \longrightarrow U^{[i]},
$$

which commutes with the action of $L$, namely

$$
d_{i}(W)=e_{1} \wedge e_{2} \wedge \cdots \wedge e_{i}
$$

where $e_{1}, e_{2}, \ldots, e_{i}$ is a basis of $W \in\left[\begin{array}{c}U \\ i\end{array}\right]$. The images under $d_{i}$ satisfy the following relations: whenever $W_{1}, W_{2}, W_{3} \in\left[\begin{array}{c}U \\ i\end{array}\right]$ are pairwise distinct with $\operatorname{dim}\left\langle W_{1}, W_{2}, W_{3}\right\rangle=i+1$ and $\operatorname{dim}\left(W_{1} \cap W_{2} \cap W_{3}\right)=i-1$, we have

$$
d_{i}\left(W_{1}\right)+d_{i}\left(W_{2}\right)+d_{i}\left(W_{3}\right)=0 .
$$


We call these the basic relations of the exterior power. By Lemma 3.1.3 in [IS02] the basic relations together with the obvious ones stating that the images are involutions, form an abstract presentation for $U^{[i]}$ in terms of generators and relations. The following lemma is a direct consequence of the basic relations of the exterior powers.

Lemma 3.1. Let $w \in U^{\#}, W \in\left[\begin{array}{c}U \\ n-1\end{array}\right], w \notin W, K_{0}$ be the stabilizer in $L$ of the direct sum decomposition

$$
U=\langle w\rangle \oplus W
$$

Put

$U^{[i]}(w)=\left\langle d_{i}(s) \mid s \in\left[\begin{array}{c}U \\ i\end{array}\right], s \sim w\right\rangle, \quad U^{[i]}(W)=\left\langle d_{i}(s) \mid s \in\left[\begin{array}{c}U \\ i\end{array}\right], s \sim W\right\rangle$,

where $\sim$ is the incidence relation in the projective geometry of $U$.

Then

(i) $K_{0} \cong L_{n-1}(2)$;

(ii) $K_{0}$ stabilizes the direct sum decomposition $U^{[i]}=U^{[i]}(w) \oplus U^{[i]}(W)$, where $U^{[i]}(w) \cong W^{[i-1]}$ and $U^{[i]}(W) \cong W^{[i]}$ as $K_{0}$-modules.

Notice that if $P_{1}$ and $P_{2}$ are the stabilizers in $L$ of $w$ and $W$, respectively, then $K_{0}=P_{1} \cap P_{2}, P_{1}=Q_{1}: K_{0}, P_{2}=Q_{2}: K_{0}$, where $Q_{1}$ is the group of transvections with centre $w, Q_{2}$ is the group of transvections with axis $W$, both $Q_{1}$ and $Q_{2}$ are elementary abelian 2-groups of rank $n-1$.

LEMMA 3.2. ([JP76]) The first cohomology group $H^{1}\left(L_{n}(2), U^{[i]}\right)$ is trivial except for the following cases where it is 1-dimensional: (a) $n=3, i=1$ or 2 ; $(b) n=4, i=2$.

Thus the semidirect product of $U^{[i]}$ and $L_{n}(2)$ with respect to the natural action possesses a unique conjugacy class of complements except for the cases (a) and (b) in Lemma 3.2 where there are exactly two classes of complements.

LEMmA 3.3. ([B78]) The second cohomology group $H^{2}\left(L_{n}(2), U^{[i]}\right)$ is trivial except for the following cases where it is 1-dimensional: (a) $n=3$, $i=1$ or $2 ;(b) n=4, i=1$ or $3 ;(c) n=5, i=1$ or 4 . 
Let $2^{n} \cdot L_{n}(2)$ denote a non-split extension by $L_{n}(2)$ of its natural module. By Lemma 3.3 and the obvious duality such extension exists only if $n=3,4$ or 5 in which case it is unique. The extension $2^{5} \cdot L_{5}(2)$ is known as the Dempwolff group.

Lemma 3.4. ([K87]) The Schur multiplier of the group $L_{n}(2)$ is trivial for $n \geq 5$ and it is of order 2 for $n=3$ and 4 .

The non-split extension $2 \cdot L_{3}(2)$ is isomorphic to the group $S L_{2}(7)$ (recall that $\left.L_{3}(2) \cong L_{2}(7)\right)$ and the extension $2 \cdot L_{4}(2)$ is isomorphic to the covering group $\widehat{A}_{8}$ of the alternating group $A_{8} \cong L_{4}(2)$.

\section{1. $n=3$}

By Lemma 3.2 there exists a unique indecomposable extension $W$ of the natural module $U$ of $L=L_{3}(2)$ by 1-dimensional module (this means that $\operatorname{dim} W=4$ and $U$ is the unique proper submodule of $W$ ). Consider the semidirect product $F=W: L$ with respect to the natural action. Then according to the standard principle $F$ contains a unique class of complements to $W=O_{2}(F)$ and if $w \in W \backslash U$ then $w$, acting by conjugation induces an outer automorphism of the group $H=U: L$ which is trivial on both $U$ and $H / U \cong L$. It follows from the transitivity of $L$ on $W_{i} \backslash U^{[i]}$ that $C_{L}(w)$ is the Frobenius group $F_{7}^{3}$ of order 21. Let $P_{1}$ and $P_{2}$ be the stabilizers in $L$ of a 1-dimensional and containing it 2-dimensional subspaces from $U$. For $i=1$ and 2 set $H_{i}=U: P_{i}, Q_{i}=O_{2}\left(H_{i}\right)$, let $T_{i}$ be a Sylow 3-subgroup in $H_{i}$ and $F_{i}=W: P_{i}$. Then

LEMмA 3.5. The following assertions hold:

(i) $H_{1} \cong 2_{+}^{1+4}: S_{3}, Q_{1}$ is the extraspecial group of plus type; $Q_{1}$ contains exactly three elementary abelian subgroups of order 8 , say $A_{1}=U$, $A_{2}$ and $A_{3} ; T_{1}$ normalizes each $A_{i}$ and acts fixed-point freely on $Q_{1} / Z\left(Q_{1}\right)$; the action of $w$ on $Q_{1}$ coincides with the unique element from Aut $Q_{1} \cong 2^{4}$ : $O_{4}^{+}(2)$ which commutes with $H_{1} / Q_{1} \cong S_{3}$; w permutes $A_{2}$ and $A_{3}$ and if $T_{1}$ is chosen so that it is normalized by $w$, then $w$ induces an outer automorphism of $N_{H_{1}}\left(T_{1}\right) \cong S_{3} \times 2$;

(ii) $H_{2} \cong 2^{4}:\left(S_{3} \times 2\right)$, so that $R_{2}:=\left[T_{2}, Q_{2}\right]$ is elementary abelian of order $2^{4} ; T_{2}$ acts fixed-point freely on $R_{2}, H_{2} /\left(R_{2} T_{2}\right) \cong 2^{2}$ together with w generate a Sylow 2-subgroup $D_{8}$ in Out $Q_{2} T_{2} \cong P \Gamma L_{2}(4) \cong S_{5}$. 
Next we discuss the structure of certain $G F(2)$-permutation modules for $L$. Let $\mathcal{P}$ is the $G F(2)$-permutation module of a group $F$ acting transitively on a set $\Omega$. Then $\mathcal{P}$ is the space of $G F(2)$-valued functions on $\Omega$, each such function being identified with its support. Then $F$ preserves on $\mathcal{P}$ a bilinear form $f$ defined by the rule that $f(A, B)$ is $|A \cap B|$ taken modulo 2 (here $A$ and $B$ are elements of $\mathcal{P}$ treated as subsets of $\Omega$ ). The the unique 1-dimensional submodule $\mathcal{P}_{c}$ of $\mathcal{P}$ consists of the two constant functions and the unique codimension 1 submodule $\mathcal{P}_{e}$ consists of the even subsets of $\mathcal{P}$ (known as the even half of $\mathcal{P}$ ). We have $\mathcal{P}_{e}=\mathcal{P}_{c}^{\perp}$ where perp is with respect to the above defined bilinear form $f$.

Lemma 3.6. Let $\mathcal{S}, \mathcal{A}$ and $\mathcal{F}$ be the $G F(2)$-permutation modules of $L$ acting on the cosets of $P_{1} \cong S_{4}, P_{1}^{\prime} \cong A_{4}$ and $F_{7}^{3}$, respectively. Then

(i) $\mathcal{S}=\mathcal{S}_{c} \oplus \mathcal{S}_{e}$ and $\mathcal{S}$ is the unique indecomposable extension of $U^{*}$ by $U$

(ii) $\mathcal{F}_{c}$ is the unique minimal submodule in $\mathcal{F}$ and $\mathcal{F}_{e} / \mathcal{F}_{c} \cong U \oplus U^{*}$;

(iii) $\mathcal{A}$ contains a submodule $\mathcal{C}$ such that $\mathcal{C} \cong \mathcal{A} / \mathcal{C} \cong \mathcal{S}$;

(iv) if $\mathcal{C}_{e}$ is the codimension 1 submodule in $\mathcal{C}$, then $\mathcal{A}_{e} / \mathcal{C}_{e}$ is indecomposable while its unique codimension 3 submodule is semi simple.

Proof. The submodule structure of $\mathcal{S}$ is well known (cf. Sections 3.2 and 3.3 in [IS02]), while the uniqueness is by Table I in [B78]. Let $T$ be a Sylow 2-subgroup in $L$. Then $|T|=8$ and $T$ acts transitively on the set of eight $F_{7}^{3}$-cosets in $L$, therefore $\mathcal{F}_{c}=C_{\mathcal{F}}(T)$ is the unique minimal, while $\mathcal{F}_{e}=\mathcal{F}_{c}^{\perp}$ is the unique maximal submodules in $\mathcal{F}$. Since $L$ acts transitively on $W_{i} \backslash U^{[i]}$ with stabilizer $F_{7}^{3}, W_{i}$ is a quotient of $\mathcal{F}$. Hence $\mathcal{F}_{e} / \mathcal{F}_{c}$ possesses both $U^{[1]}$ and $U^{[2]}$ as quotients and (ii) follows. (iii) is quite clear. The dual $W_{i}^{*}$ of $W_{i}$ is an indecomposable extension of the 1-dimensional module by $U^{[3-i]}=\left(U^{[i]}\right)^{*}$. If $L$ would have a 7 -orbit on the vectors in $W_{i}^{*}$, the latter would be a quotient of $\mathcal{S}^{(3-i)}$, contrary to (i). Hence $L$ is transitive on the non-zero vectors in $W_{i}^{*}$ and the latter is a quotient of $\mathcal{A}^{(3-i)}$. If the corresponding kernel would contain $\mathcal{C}^{(3-i)}$, by (iii) this again would be a contradiction with (i). Hence (iv) follows.

Notice that $W$ is a quotient of $\mathcal{F}$ while $W^{*}$ is a quotient of $\mathcal{A}$.

LEMMA 3.7. $H^{1}\left(L_{3}(2), W^{*}\right)$ is trivial. 
Proof. To establish the claim it is sufficient to show the non-existence of an indecomposable extension of 1-dimensional module by $W$. Suppose that $X$ is such an extension and $\varphi: X \rightarrow W$ is the canonical homomorphism. As stated in the paragraph before Lemma 3.5, $L$ acts transitively on $W \backslash U$ with stabilizer $F_{7}^{3}$. Since the latter group has no subgroups of index $2, L$ has two orbits on $\varphi^{-1}(W \backslash U)$. Each of these orbits is of length 8 and generates $X$ (since $X$ is indecomposable). Therefore $X$ is a quotient of $\mathcal{F}$ over a 3-dimensional irreducible submodule, which is impossible by Lemma 3.6 (ii).

LEMmA 3.8. With $H=U: L$ as above let $\widehat{H}$ be the covering group of $H$ and for $X \leq H$ let $\widehat{X}$ denote the preimage of $X$ in $\widehat{H}$. Let $L_{1}$ and $L_{2}$ be representatives of two classes of complements to $U$ in $H$. Then

(i) $\widehat{L}_{1} \cong \widehat{L}_{2} \cong S L_{2}(7)$;

(ii) $Z(\widehat{H})$ is elementary abelian of order 4 and its non-identity elements $c, z_{1}, z_{2}$ are such that

(a) $\left\langle z_{i}\right\rangle=Z\left(\widehat{L}_{i}\right)$ for $i=1,2$;

(b) $\widehat{U}=\left\langle z_{1}\right\rangle \oplus[\widehat{U}, \widehat{H}]$ (where $[\widehat{U}, \widehat{H}] \cong W^{*}$ is the indecomposable extension of $\langle c\rangle$ by $U)$.

Proof. By (3.4) the Schur multiplier of $L_{3}(2) \cong H / \mathrm{O}_{2}(H)$ is of order 2 and of course $\mathrm{O}_{2}(H)$ is a 2-group. This immediately implies that $Z(\widehat{H})$ is a 2-group and if $\widehat{U}$ is the preimage of $U$ in $\widehat{H}$ then $\widehat{U}=O_{2}(\widehat{H})$. We claim that $\widehat{U}$ is an elementary abelian 2-group. Let $\widehat{A}$ be the commutator subgroup of $\widehat{U}$ and $\widehat{B}$ be the subgroup of $\widehat{U}$ generated by the squares of the elements of $\widehat{U}$. Suppose that $\widehat{A}$ and/or $\widehat{B}$ are non-trivial. Let $\widehat{A}_{1}$ and $\widehat{B}_{1}$ be subgroups of index 2 in $\widehat{A}$ and $\widehat{B}$, respectively. Then the mappings:

$$
(\widehat{u}, \widehat{v}) \longmapsto[\widehat{u}, \widehat{v}] \widetilde{A}_{1} / \widehat{A}_{1}
$$

and

$$
\widehat{u} \longmapsto \widehat{u} \widehat{B}_{1} / \widehat{B}_{1}
$$

define a bilinear alternating and quadratic forms on $U=\widehat{U} / Z(\widehat{H})$, respectively and these forms are invariant under $L$. Since $L$ induces the full linear group of $U$, there are no such non-zero forms and the claim follows. By Lemma $3.2 H^{1}(L, U)$ is 1-dimensional, hence $[\widehat{U}, \widehat{H}] \cap Z(\widehat{H})$ is of order (at most) 2 , generated by an element $c$, say. Then $\widehat{U} /\langle c\rangle$ is decomposable and we can factor out a complement to $Z(\widehat{H}) /\langle c\rangle$ in $\widehat{U} /\langle c\rangle$. The resulting group 
is a perfect central extension of $H / U \cong L_{3}(2)$, i.e., a quotient of $S L_{2}(7)$. Therefore $Z(\widehat{H})$ is of order at most four. Considering the semidirect product of $W^{*}$ and $S L_{2}(7)$ with the centre of the latter acting trivially, we see that the bound is attained. Since $\widehat{H}$ is the universal covering group, an outer automorphism of $H$ which sends $L_{1}$ onto $L_{2}$ extends to an automorphism of $\widehat{H}$, which gives (i). Suppose that $Z\left(\widehat{L}_{1}\right)=Z\left(\widehat{L}_{2}\right)$. Then $\widehat{H} / Z\left(L_{1}\right)$ is a semidirect product of $W^{*}$ and $L_{3}(2)$ with two classes of complements, whose existence contradicts Lemma 3.7, hence (ii) follows.

As a consequence of Lemma 3.8 and its proof we have the following

LEMMA 3.9. Let $F$ be a group such that $Z(F)$ is or order $2, F / Z(F) \cong$ $H \cong U: L$ and $\mathrm{O}_{2}(F)$ is isomorphic to $W^{*}$ as a module for $F / O_{2}(F) \cong$ $L_{3}(2)$. Let $K_{1}$ and $K_{2}$ be the preimages in $F$ of representatives of the two classes of complements to $U$ in $H$. Then (up to renumbering) $K_{1} \cong$ $L_{3}(2) \times 2$ while $K_{2} \cong S L_{2}(7)$. In particular $F$ is determined uniquely up to isomorphism as the semidirect product of $W^{*}$ and $O^{2}\left(K_{1}\right)$ with respect to the natural action.

Proof. Since $F$ is a perfect extension of $H$ by a centre of order 2, in terms of Lemma 3.8 there is a homomorphism of $\widehat{H}$ onto $F$, whose kernel is $\left\langle z_{i}\right\rangle$ for $i=1$ or 2 , hence the result.

LEMma 3.10. $H^{1}\left(L_{3}(2), \mathcal{S}_{e}\right)$ is 1-dimensional.

Proof. Notice first that $\mathcal{S}_{e}$ is self-dual. By Lemma 3.6 (iv) $\mathcal{A}_{e} / \mathcal{C}_{e}$ is an indecomposable extension of a 1-dimensional module by $\mathcal{S}_{e}$, hence $H^{1}\left(L_{3}(2), \mathcal{S}_{e}\right)$ is at least 1-dimensional. Suppose it is more than 1-dimensional. Then there exists an indecomposable extension $Y$ of a 1-dimensional module by $\mathcal{A}_{e} / \mathcal{C}_{e}$. It is easy to see that the latter is a quotient of $\mathcal{A}$, i.e., contains a generating orbit of length 14 with stabilizer $A_{4}$. Since the latter group has no subgroups of index $2, Y$ must also be a quotient of $\mathcal{A}$, which contradicts Lemma 3.6.

LEMMA 3.11. Let $Y$ be a group, such that $Y / O_{2}(Y) \cong L_{3}(2), O_{2}(Y)$ is elementary abelian, isomorphic to the unique indecomposable extension $\mathcal{S}_{e}$ of $U^{*}$ by $U$ (cf. Lemma $3.6(\mathrm{i})$ ). Suppose further that (identifying $U^{*}$ with the corresponding submodule in $\left.\mathrm{O}_{2}(Y)\right) Y / U^{*} \cong H=U:$ L. Let $M_{1}$ and $M_{2}$ be the preimages in $Y$ of representatives of two classes of complements to $U$ in $H$. Then, up to renumbering, $M_{1} \cong U^{*}: L$ (semidirect product), while $M_{2} \cong 2^{3} \cdot L_{3}(2)$ (non-split extension). 
Proof. Let $\widehat{Y}$ be the largest group subject to the following properties: (1) $\widehat{Y} / O_{2}(\widehat{Y}) \cong L_{3}(2) ;(2) O_{2}(\widehat{Y})$ is an elementary abelian 2-group; (3) $\widehat{Y}$ possesses a homomorphism $\varphi$ onto $H=U: L$ and if $X$ is the kernel of $\varphi$ then $J$ is a direct sum of $\widehat{Y} / O_{2}(\widehat{Y})$-modules, each isomorphic to $U^{*}$; (4) if $T$ is a subgroup in $\widehat{Y}$ such that $T J=\widehat{Y}$, then $T=\widehat{Y}$. We prove that such largest group exists by establishing a bound on its order and by showing that the bound is attained. Let $R$ be the smallest submodule in $O_{2}(\widehat{Y})$ such that $O_{2}(\widehat{Y}) / R$ is a direct sum of copies of $U^{*}$. Then $R$ is an indecomposable extension of the direct sum of, say $i$ copies of $U^{*}$ by $U$. Since there is a unique indecomposable extension of $U^{*}$ by $U$ (cf. Lemma 3.6 (i)), we have $i \leq 1$. Next consider $\widehat{Y} / R$ which is by (3) and (4) must be a non-split extension of the direct sum of, say $j$ copies of $U^{*}$ by $L_{3}(2)$. Since $H^{2}\left(L_{3}(2), U^{*}\right)$ is 1-dimensional (cf. Lemma 3.3), we have $j \leq 1$ and the bound is established. Now consider the semidirect product of $\mathcal{S}_{e}$ and $\widehat{M}_{1} \cong 2^{3} \cdot L_{3}(2)$ with $O_{2}\left(\widehat{M}_{1}\right)$ acting trivially and $\widehat{M}_{1} / O_{2}\left(\widehat{M}_{1}\right)$ acting in the natural way, we observe that the resulting group satisfies (1)-(4) and its order attains the bound, hence it is the group $\widehat{Y}$ we are after. By the maximality property every automorphism of $H$ extends to an automorphism of $\widehat{Y}$. Hence the preimage of every $L_{3}(2)$-complement from $H$ does not split. Let $\widehat{M}_{1}, \widehat{M}_{2}$ be subgroups in $\widehat{Y}$, both isomorphic to $2^{3} \cdot L_{3}(2)$ such that their images in $H$ are not conjugate. We claim that $O_{2}\left(\widehat{M}_{1}\right) \neq O_{2}\left(\widehat{M}_{2}\right)$. Indeed, otherwise $\widehat{Y} / O_{2}\left(\widehat{M}_{1}\right)$ would be a semidirect product of $\mathcal{S}_{e}$ and $L_{3}(2)$ with four classes of complements contrary to Lemma 3.10. Therefore $J=V_{1} \oplus V_{2}$, where $V_{i}=O_{2}\left(V_{i}\right)$ for $i=1,2$ and $V_{3}=J \cap R$ is the third non-trivial submodule in $J$. Now the group $Y$ in the hypothesis of the lemma is the quotient of $\widehat{Y}$ over $V_{i}$ for $i=1$ or 2 and the result follows.

\section{2. $n=4$}

Let $L=L_{4}(2), U$ be a natural module of $L, \Omega$ be a set of size eight of which $L \cong A_{8}$ is the alternating group and $\mathcal{E}$ be the $G F(2)$-permutation module of $L$ on $\Omega$. Let $P_{1}$ and $P_{2}$ be the stabilizers in $L$ of a 1 -subspace $\langle w\rangle$ and a 3-subspace $W$ in $U$, respectively, where $w \in U \backslash W$, so that $P_{1} \cong P_{2} \cong 2^{3}: L_{3}(2)$.

Lemma 3.12. The group $L \cong L_{4}(2)$ contains exactly three conjugacy classes of subgroups isomorphic to $L_{3}(2)$. Representatives $K_{0}, K_{1}$ and $K_{2}$ of these classes can be chosen in such a way that

(i) $K_{0}, K_{i} \leq P_{i}$ for $i=1,2$; 
(ii) $K_{0}$ is a Levi complement in $P_{1}$ and $P_{2}$, and it is the stabilizer of the direct sum decomposition $U=\langle w\rangle \oplus W$;

(iii) both $K_{1}$ and $K_{2}$ act indecomposably on $U$;

(iv) $K_{0}$ acts transitively on $\Omega$ while $K_{i}$ for $i=1$ or 2 stabilizes a point in $\Omega$ and acts transitively on the remaining points, so that $K_{i}<A_{7}<$ $A_{8} \cong L$;

(v) there is an outer automorphism $\tau$ of $L$ such that $K_{i}^{\tau}=K_{3-i}$ for $i=1,2, \tau$ normalizes $K_{0}$ and $\left\langle K_{0}, \tau\right\rangle \cong P G L_{2}(7)$.

Proof. This is all well known and easy to verify. If we treat $L$ as the alternating group $A_{8}$ then the automorphism $\tau$ in $(\mathrm{v})$ is induced by an odd permutation and $\langle L, \tau\rangle \cong S_{8}$. If we consider $L$ as the linear group $L_{4}(2)$, then $\tau$ is induced by a conjugate of the inverse-transpose automorphism, it maps the natural module $U$ onto its dual and normalizes $U^{[2]}$.

LEMMA 3.13. Let $\widehat{L} \cong 2 \cdot L_{4}(2)$ be the covering group of $L$ and for a subgroup $X$ of $L$ let $\widehat{X}$ denote its preimage in $\widehat{L}$. Then in terms of Lemma 3.12 the following assertions hold

(i) $\widehat{K}_{1} \cong \widehat{K}_{2} \cong S L_{2}(7), \widehat{K}_{0} \cong L_{3}(2) \times 2$;

(ii) for $i=1$ and $2 O_{2}\left(\widehat{P}_{i}\right)$ is the indecomposable extension of $Z(\widehat{L})=$ $Z\left(\widehat{K}_{i}\right)$ by $O_{2}\left(P_{i}\right) \cong 2^{3}$.

Proof. The result can be deduced from the well known fact (cf. [K87]) that the involutions in $L$ acting fixed-point freely on $\Omega$ lift to involutions in $\widehat{L}$ while the products of pairs of commuting transpositions lift to elements of order 4 .

The exterior square $U^{[2]}$ of $U$ is the natural module for $L$, treated as the simple orthogonal group $\Omega_{6}^{+}(2)$. On the other hand $U^{[2]} \cong \mathcal{E}_{e} / \mathcal{E}_{c}$. This shows that the automorphism $\tau$ as in Lemma 3.12 extends to an automorphism $\delta$ of the semidirect product

$$
D=U^{[2]}: L .
$$

It is well known that $\mathcal{E}$ is uniserial and in view of Lemma 3.2 this means that $\mathcal{E} / \mathcal{E}_{c}$ is the unique indecomposable extension of $U^{[2]}$ by a 1 -dimensional module and $\mathcal{E}_{e}$ is the unique indecomposable extension of a 1-dimensional module by $U^{[2]}$. Thus $\left(\mathcal{E} / \mathcal{E}_{c}\right): L$ contains $D$ with index 2 and an element 
from $\left(\mathcal{E} / \mathcal{E}_{c}\right) \backslash\left(\mathcal{E}_{e} / \mathcal{E}_{c}\right)$ induces an outer automorphism $\sigma$ of $D$ permuting two classes of $L_{4}(2)$-complements and acting trivially on $D / O_{2}(D)$. There are two orbits on such elements with stabilizers isomorphic to $A_{7}$ (the stabilizer of an element in $\Omega$ ) and $\left(S_{3} \times S_{5}\right)^{+}$(the stabilizer of a 3 -subset in $\Omega$ ), respectively. By Lemma 3.12 (iv) we can chose such an element to commute with $K_{i}$ for $i=1$ or 2 , but not for $i=0$. We record this in the following

Lemma 3.14. The semidirect product $D=U^{[2]}:$ L contains two classes of $L_{4}(2)$-complements. If $L^{*}$ is a complement not in the class of $L$ then e $i$ ther $L \cap L^{*} \cong A_{7}$ or $L \cap L^{*} \cong\left(S_{3} \times S_{5}\right)^{+}$, in particular $K_{0} \not \leq L^{*}$.

Let $q$ denote the non-degenerate quadratic form (of plus type) on $U^{[2]}$ preserved by $L \cong \Omega_{6}^{+}(2)$.

Lemma 3.15. The group $L=L_{4}(2)$ has two orbits, $\Sigma_{1}$ and $\Sigma_{2}$ on the set of non-zero vectors in $U^{[2]}$, such that

(i) $\left|\Sigma_{1}\right|=35$, if $\alpha \in \Sigma_{1}$, then $q(\alpha)=0, L(\alpha) \cong 2^{4}:\left(S_{3} \times S_{3}\right) \cong 2^{4}$ : $\Omega_{4}^{+}(2)$ is the stabilizer of a 2-subspace in $U$;

(ii) $\left|\Sigma_{2}\right|=28$, if $\alpha \in \Sigma_{2}$, then $q(\alpha)=1, L(\alpha) \cong S_{6} \cong S p_{4}(2)$ is the stabilizer of a non-singular symplectic form on $U$.

Let $d_{2}$ be the mapping from the set $\left[\begin{array}{c}U \\ 2\end{array}\right]$ of 2-subspaces in $U$ into $U^{[2]}$ as defined before Lemma 3.1 (so that $\Sigma_{1}$ is the image of $d_{2}$ ) and adopt the notation of that lemma.

LEMMA 3.16. The following assertions hold

(i) $U^{[2]}(w)=C_{U^{[2]}}\left(O_{2}\left(P_{1}\right)\right)$, as a module for $P_{1} / O_{2}\left(P_{1}\right)$ the subspace $U^{[2]}(w)$ is isomorphic to $U /\langle w\rangle$ and it is the unique non-trivial $K_{1^{-}}$submodule in $U^{[2]}$;

(ii) $U^{[2]}(W)=C_{U^{[2]}}\left(O_{2}\left(P_{2}\right)\right)$, as a module for $P_{2} / O_{2}\left(P_{2}\right)$ the subspace $U^{[2]}(W)$ is isomorphic to $W^{*}$ and it is the unique non-trivial $K_{2}$ submodule in $U^{[2]}$;

(iii) $K_{0}$ is the stabilizer in $L$ of the direct sum decomposition $U^{[2]}=$ $U^{[2]}(w) \oplus U^{[2]}(W)$, furthermore $U^{[2]}(w) \cong W$, while $U^{[2]}(W) \cong W^{*}$ (as $K_{0}$-modules).

Proof. Again this is well known and easy to verify. 
Let us classify up to isomorphism the 2-constrained groups $\widetilde{D}$ of the form $2_{+}^{1+6} \cdot L_{4}(2)$. Thus $O_{2}(\widetilde{D})$ is extraspecial of order $2^{7}$ and of plus type, $\widetilde{D} / O_{2}(\widetilde{D}) \cong L_{4}(2)$ and $C_{\widetilde{D}}\left(O_{2}(\widetilde{D})\right) \leq O_{2}(\widetilde{D})$. Then

$$
\widetilde{D} / Z(\widetilde{D}) \cong D \cong U^{[2]}: L_{4}(2)
$$

is determined uniquely up to isomorphism and in order to accomplish our goal we calculate the Schur multiplier of $D$. Let $\sigma$ and $\delta$ be the above defined outer automorphisms of $D$. Recall that $\sigma$ permutes the classes of $L_{4}(2)$-complements and commutes with $D / O_{2}(D)$, while $\delta$ normalizes such a complement and induces on it an outer automorphism. It is easy to check that Aut $D=\langle\operatorname{Inn} D, \sigma, \delta\rangle$ and Out $D \cong 2^{2}$. Clearly $\delta$ and $\sigma$ lift to automorphisms of the covering group of $D$ and we denote these liftings by the same letters.

LEMMA 3.17. The Schur multiplier of $D$ is elementary abelian of order 8. If $\widehat{D}$ is the covering group of $D$, then $Z(\widehat{D})$ is generated by elements $c$, $\pi, z$, such that

(i) $C=\langle c\rangle$ is the commutator subgroup of $\mathrm{O}_{2}(\widehat{D})$;

(ii) $\left(Z(\widehat{D}) \cap\left[O_{2}(\widehat{D}), \widehat{D}\right]\right) / C=\langle c, \pi\rangle / C$;

(iii) if $L_{4}(2) \cong L \leq D$ and $\widehat{L}$ is the preimage of $L$ in $\widehat{D}$, then $\langle z\rangle=$ $Z([\widehat{L}, \widehat{L}])$ (independently on the choice of the complement $L)$;

(iv) $\delta \sigma$ centralizes $Z(\widehat{D})$ while both $\sigma$ and $\delta$ centralize $\langle c, z\rangle$ and map $\pi$ onto $\pi c$.

Proof. Since $L$ preserves on $U^{[2]}$ a unique non-zero alternating form (namely the one associated with $q$ ), the commutator subgroup $C$ of $O_{2}(\widehat{D})$ is of order at most 2. Since $H^{1}\left(L_{4}(2), U^{[2]}\right)$ is 1-dimensional, we have (ii). Then $Z(\widehat{D}) /\langle c, \pi\rangle$ is complemented in $O_{2}(\widehat{D}) /\langle c, \pi\rangle$ and the quotient over the complement is a perfect central extension of $D / O_{2}(D) \cong L_{4}(2)$. Now to establish (iii) it remains to show that whenever $L_{1}$ and $L_{2}$ are two $L_{4}(2)$-complements in $D$ and $\widehat{L}_{1}, \widehat{L}_{2}$ are their preimages in $\widehat{D}$, we have $Z\left(\left[\widehat{L}_{1}, \widehat{L}_{1}\right]\right)=Z\left(\left[\widehat{L}_{2}, \widehat{L}_{2}\right]\right)$. If $L_{1}$ and $L_{2}$ are in the same class, this is obvious, otherwise by Lemma 3.14 we can assume that $L_{1} \cap L_{2} \cong A_{7}$. Since the preimage of $A_{7}$ in the covering group of $A_{8}$ does not split, (iii) follows.

In order to show that the bound 8 on the order of $Z(\widehat{D})$ is attained and to determine the action of Out $D$ on $Z(\widehat{D})$ we construct a group $E$ such that $E^{\prime} \cong \widehat{D}$ has centre of order 8 and $E / Z\left(E^{\prime}\right) \cong$ Aut $D$. The construction 
is inspired by the following observation. Let $R \cong O_{8}^{+}(2) \cong \Omega_{8}^{+}(2): 2$ act on its natural module $V$ and $v \in V^{\#}$ be an isotropic vector. Then

$$
R(v) \cong 2^{6}: S_{8} \cong\langle D, \delta\rangle
$$

Let $S$ be a complement to $O_{2}(R(v))$ in $R(v)$, which is not a Levi complement (i.e., $S$ does not stabilize maximal totally singular subspaces in $V$ ). Then $S$ acts irreducibly on $v^{\perp} /\langle v\rangle$ and has an orbit of length 8 on the set of isotropic vectors outside $v^{\perp}$ (recall that $V: R \cong$ Aut $2_{+}^{1+8}$ ).

Let $Q$ be a group generated by elements $a_{i}, 1 \leq i \leq 8$ and $c$ subject to the following relations:

$$
a_{i}^{2}=c^{2}=\left[a_{i}, c\right]=1, \quad\left[a_{i}, a_{j}\right]=c, \quad 1 \leq i, j \leq 8, i \neq j .
$$

Then $Q$ is extraspecial of order $2^{9}$ and of plus type. Notice that every element of $Q$ possesses a unique presentation of the form $a_{i_{1}} a_{i_{2}} \cdots a_{i_{m}} c^{x}$, where $0 \leq m \leq 8,1 \leq i_{1}<i_{2}<\cdots<i_{m} \leq 8, x \in\{0,1\}$. The symmetric group $S \cong S_{8}$ acts naturally on $\left\{a_{i} \mid 1 \leq i \leq 8\right\}$ and this action extends to an automorphism group of $Q$. There is a natural $S$-homomorphism $\varphi: Q \rightarrow \mathcal{E}$, where $\mathcal{E}$ is the 8-dimensional $G F(2)$-permutation module of $S$.

The centralizer $T$ of $S^{\prime} \cong A_{8}$ in $Q$ is elementary abelian of order $2^{2}$ with the non-identity elements

$$
c, \pi=a_{1} a_{2} a_{3} \cdots a_{8}, \pi c=a_{2} a_{1} a_{3} \cdots a_{8}
$$

and $\varphi(T)=\mathcal{E}_{c}$. If $s \in S \backslash S^{\prime}$ then $\pi^{s}=\pi c$. It follows from the basic properties of extraspecial groups that if $P=C_{Q}(T)$ then $P$ has index 2 in $Q$ and $\pi^{q}=\pi c$ for $q \in Q \backslash P$. Furthermore, $\varphi(P)=\mathcal{E}_{e}$. Let $\widehat{S} \cong 2 \cdot S_{8}$ be a representation group of $S$ (so that $\widehat{S} / Z(\widehat{S}) \cong S_{8}$ and the commutator subgroup of $\widehat{S}$ is the covering group of $\left.A_{8}\right)$. Let $E$ be the semidirect product of $Q$ and $\widehat{S}$ with $Z(\widehat{S})$ acting trivially and $\widehat{S} / Z(\widehat{S}) \cong S$ acting in the above defined way. Then the commutator subgroup $E^{\prime}$ of $E$ is $P \widehat{S}^{\prime}$ has index 4. The centre of $E^{\prime}$ is elementary abelian of order 8 generated by $c, \pi$ and $z$, where the latter element is the generator of $Z(\widehat{S}) ; E^{\prime} / Z\left(E^{\prime}\right) \cong D$ and the elements $s$ and $q$ as above induce on $D$ the automorphisms $\delta$ and $\sigma$, respectively. Now the result is clear.

As a consequence of Lemma 3.17 and its proof we have the following.

LEMMA 3.18. There are two isomorphism classes of 2-constrained groups of the form $2_{+}^{1+6} . L_{4}(2)$ with representatives $\widetilde{D}_{1}=\widehat{D} /\langle\pi, z\rangle$ and 
$\widetilde{D}_{2}=\widehat{D} /\langle\pi, z c\rangle$, where $O_{2}\left(\widetilde{D}_{i}\right)$ is complemented in $\widetilde{D}_{i}$ for $i=1$ but not for $i=2$. The automorphism $\delta \sigma$ of $D$ extends to automorphisms of $\widetilde{D}_{1}$ and $\widetilde{D}_{2}$ and (abusing the notation)

$$
\left\langle\widetilde{D}_{1}, \delta \sigma\right\rangle / Z\left(\widetilde{D}_{1}\right) \cong\left\langle\widetilde{D}_{2}, \delta \sigma\right\rangle / Z\left(\widetilde{D}_{2}\right) \cong 2^{6} \cdot S_{8}
$$

( a non-split extension of $U^{[2]}$ by $S_{8} \cong O_{6}^{+}(2)$ ).

\section{$\S 4$. The vertex stabilizer}

As we have already mentioned, within the proof of Trofimov's theorem the structure of chief factors of the vertex stabilizer in a locally projective action was established (cf. [Tr03a]). In the case of type $(n, 2)$ action which is strictly 2-arc-transitive of collineation shape all non-trivial chief factors inside $G_{1}(x)$ are exterior powers of the natural module $U$ for $L=G(x) / G_{1}(x) \cong L_{n}(2)$. More precisely the following statement holds.

Proposition 4.1. Let the action of $G$ on $\Gamma$ be locally projective of type $(n, 2), n \geq 3$. Suppose further that the action is strictly 2-arc-transitive of collineation shape. Then one of the following holds:

(i) $G_{1}(x)=1$;

(ii) $G_{2}(x)=1, G_{1}(x) \cong U^{[n-2]}$ and $G(x)$ splits over $G_{1}(x)$;

(iii) $n=3, G_{3}(x)=1, G_{1}(x) / G_{2}(x) \cong U,\left|G_{2}(x)\right|=2, G_{1}(x)$ is elementary abelian isomorphic to the direct sum of $U$ and a 1-dimensional trivial module, and $G(x)$ splits over $G_{1}(x)$;

(iv) $n=4, G_{1}(x) / G_{2}(x) \cong U^{[2]}, G_{2}(x) / G_{3}(x) \cong U,\left|G_{3}(x)\right|=2, G_{4}(x)=$ 1

(v) $n=4, G_{1}(x) / G_{2}(x) \cong U^{[2]}, G_{2}(x) / G_{3}(x) \cong U, G_{3}(x) \cong U^{[3]}$, $G_{4}(x)=1$

(vi) $n=5, G_{1}(x) / G_{2}(x) \cong U^{[3]}, G_{2}(x) / G_{3}(x) \cong U^{[2]}, G_{3}(x) / G_{4}(x) \cong U$, $G_{4}(x) \cong U^{[4]}, G_{5}(x)=1$.

Proof. The case (iii) where $n=3$ was accomplished already in [Tim84]. The cases (iv), (v) where $n=4$ proved in [Tr00] and the case (vi) where $n=5$ - in [Tr01]. Finally in [Tr03b] it was shown that $G_{2}(x)=1$ for $n \geq 6$. For (ii) see $[\operatorname{Tr} 91 \mathrm{~b}]$.

We will use the following easy methods of identifying rank 2 amalgams. 
Lemma 4.2. Let $\mathcal{A}=\left\{G^{[0]}, G^{[1]}\right\}$ and $\widetilde{\mathcal{A}}=\left\{\widetilde{G}^{[0]}, \widetilde{G}^{[1]}\right\}$ be amalgams, such that

$$
\left[G^{[1]}: G^{[0]} \cap G^{[1]}\right]=\left[\widetilde{G}^{[1]}: \widetilde{G}^{[0]} \cap \widetilde{G}^{[1]}\right]=2 .
$$

Suppose there is an isomorphism $\varphi$ of $G^{[0]}$ onto $\widetilde{G}^{[0]}$ and elements $t \in$ $G^{[1]} \backslash G^{[0]}, \widetilde{t} \in \widetilde{G}^{[1]} \backslash \widetilde{G}^{[0]}$, such that

(i) $\varphi\left(G^{[0]} \cap G^{[1]}\right)=\widetilde{G}^{[0]} \cap \widetilde{G}^{[1]}$;

(ii) for every $s \in G^{[0]} \cap G^{[1]}$ the equality $\varphi\left(t^{-1} s t\right)=\widetilde{t}^{-1} \varphi(s) \widetilde{t}$ holds;

(iii) $\varphi\left(t^{2}\right)=\widetilde{t}^{2}$.

Then $\mathcal{A}$ and $\tilde{\mathcal{A}}$ are isomorphic.

Proof. The mapping which coincides with $\varphi$ on $G^{[0]}$ and sends $t$ onto $\widetilde{t}$ extends in the obvious way to the required isomorphism of $\mathcal{A}$ onto $\widetilde{\mathcal{A}}$.

By Lemma 4.2 the isomorphism type of $\mathcal{A}=\left\{G^{[0]}, G^{[1]}\right\}$ is determined by the choice of

(a) $G^{[0]}$ up to isomorphism;

(b) $G^{[0]} \cap G^{[1]} \leq G^{[0]}$ up to conjugation in the automorphism group of $G^{[0]}$;

(c) an automorphism $\psi$ of $G^{[0]} \cap G^{[1]}$ (induced by an element $t \in G^{[0]} \backslash$ $\left.G^{[1]}\right)$, which does not normalize subgroups in $G^{[0]} \cap G^{[1]}$ normal in $G^{[0]}$ and whose square is an inner automorphism;

(d) an element $q \in G^{[0]} \cap G^{[1]}$ centralized by $\psi$ (such that $t^{2}=q$ ).

It is clear that the choice of $t$ and hence of $\psi$ is not unique. For instance we can change $t$ by $t g$ for any $g \in G^{[0]} \cap G^{[1]}$ (this effects in multiplying $\psi$ by the inner automorphism induced by $g$ ). Similarly we can multiply $\psi$ by any automorphism of $G^{[0]} \cap G^{[1]}$, which extends to an automorphism of $G^{[0]}$. We formulate this in the following lemma.

Lemma 4.3. The automorphism $\psi$ in (c) above can be chosen up to multiplication by elements from the image I of $N_{\text {Aut } G^{[0]}}\left(G^{[0]} \cap G^{[1]}\right)$ in $A=$ Aut $G^{[0]} \cap G^{[1]}$. Choosing $\psi$ from different cosets of $I$ in $A$ one gets nonisomorphic amalgams. 
§5. $G_{1}(x)=1$

The case $G_{1}(x)=1$ was settled for locally projective actions of all types independently in Theorem 1.1 from [Ch99] and Lemma 2.1 from [IP98].

In the considered situation (case (i) in Proposition 4.1) $G(x, y)$ is the semidirect product of $L_{n-1}(2)$ and its natural module. By Lemma 3.2 this group is complete unless $n=4$. Thus either $G\{x, y\} \cong G(x, y) \times 2$ and $\mathcal{A} \cong \mathcal{A}\left(A G L_{n}(2), K_{2^{n}}\right)$ or $n=4, O_{2}(G\{x, y\})$ is the unique indecomposable extension of the natural module of $L_{3}(2)$ by the 1-dimensional module and $G\{x, y\}$ splits over $O_{2}(G\{x, y\})$. In this case $\mathcal{A} \cong \mathcal{A}\left(M_{23}, \Gamma\left(M_{23}\right)\right)$, where $\Gamma\left(M_{23}\right)$ is the derived graph of the rank $4 P$-geometry related to $M_{23}$.

§6. $G_{2}(x)=1$

In this section we consider the case (ii) in Proposition 4.1.

Proposition 6.1. The following assertions hold:

(i) $G(x, y)=Z A B K$, where $K \cong L_{n-1}(2)$, while $A, B$ and $Z$ are elementary abelian 2-groups of rank $n-1, n-1$ and $(n-1)(n-2) / 2$, respectively, normalized by $K$, furthermore

(a) $A$ and $B$ are isomorphic to the dual $W^{*}$ of the natural module of $K$, while $Z$ is isomorphic to the exterior square of $W^{*}$;

(b) $Z$ is the centre of $Q:=O_{2}(G(x, y))=Z A B$;

(c) if $\varphi_{X}: X \rightarrow\left(W^{*}\right)^{[\alpha]}$ is the isomorphism commuting with the action of $K$ for $X=A, B$ or $Z$ with $\alpha=1,1$ or 2 , respectively, and $d_{2}:\left[\begin{array}{c}W^{*} \\ 2\end{array}\right] \rightarrow\left(W^{*}\right)^{[2]}$ is as define before Lemma 3.1, then for $a \in A, b \in B$ we have $[a, b]=1$ whenever $\varphi_{A}(a)=\varphi_{B}(b)$ and

$$
[a, b]=\varphi_{Z}^{-1}\left(d_{2}^{-1}\left\langle\varphi_{A}(a), \varphi_{B}(b)\right\rangle\right)
$$

otherwise;

(ii) the permutation of $Z \cup A \cup B \cup K$, which centralizes $Z \cup K$ and maps $a \in A$ onto $\varphi_{B}^{-1}\left(\varphi_{A}(a)\right) \in B$ extends to a unique automorphism $\tau_{0}$ of $G(x, y)$;

(iii) if $C=\left\langle a b \mid a \in A, b \in B, \varphi_{A}(a)=\varphi_{B}(b)\right\rangle$, then

(d) $\{A Z / Z, B Z / Z, C Z / Z\}$ is the complete set of proper $K Z / Z$-submodules in $Q / Z$; 
(e) $C$ is a direct sum of irreducible $K$-submodules if and only if $n=$ 3

(iv) if $n \geq 6$ then Out $G(x, y)$ is of order 2 , generated by the image of $\tau_{0}$.

Proof. In the considered situation $G(x)$ is the semidirect product with respect to the natural action of $M \cong L_{n}(2)$ and the exterior square $\left(U^{*}\right)^{[2]} \cong$ $U^{[n-2]}$ of the dual natural module $U$ of $M \cong L$. Let $\iota: \Gamma(x) \rightarrow U^{\#}$ be the bijection commuting with the action of $M$. Let $w=\iota(y), W$ be a hyperplane in $U$ disjoint from $w$ and $K$ be the stabilizer in $M$ of the direct sum decomposition $U=\langle w\rangle \oplus W$. Put $A=O_{2}(M \cap G(x, y))$, so that $M \cap G(x, y)=A K$ and $A \cong W^{*}$ (as $K$-modules). By Lemma 3.16 (iii) $K$ is the stabilizer in $M$ of the direct sum decomposition

$$
U^{[n-2]} \cong G_{1}(x)=Z \oplus B \cong U^{[n-2]}(W) \oplus U^{[n-2]}(w) .
$$

By Lemma 3.1 (i) $Z \cong W^{[n-3]}=\left(W^{*}\right)^{[2]}$ and $B \cong W^{[n-2]} \cong W^{*}$ (as $K$ modules). Now (a) follows while (b) and (c) are easy to deduce from the known action of $M$ on $G_{1}(x)$. So (i) is established.

Now (ii) is immediate from (i). Since $a b a^{\prime} b^{\prime}=a a^{\prime} b b^{\prime}\left[b, a^{\prime}\right]$ and by (c) $\left[b, a^{\prime}\right]$ is non-identity if $\varphi_{A}\left(a^{\prime}\right) \neq \varphi_{B}(b)$, the set of generators of $C$ (together with the identity element) is not closed under multiplication and (e) is rather clear.

Suppose now that $n \geq 6$ and let $\tau$ be an automorphism of $G(x, y)$. Then by Lemma 3.2 every complement to $Q$ in $G(x, y)$ is a conjugate of $K$. Hence, adjusting $\tau$ by an inner automorphism we can assume that $\tau$ normalizes $K$. Since $G(x, y)$ contains chief factors isomorphic to $W^{*}$ and not to $W, \tau$ can't perform an outer automorphism of $K$ and hence $\tau$ can be adjusted to centralize $K$. Since $Z$ is the centre of $Q$ is it normalized by $\tau$ and since $Z \cong\left(W^{*}\right)^{[2]}$ is absolutely irreducible $K$-module (and $\tau$ centralizes $K), \tau$ must centralize $Z$. We claim that $\tau$ either normalizes $A$ or maps it onto $B$. Indeed, let $a \in A^{\#}$. Then $C_{K}(a)$ acts fixed-point freely on $Z$ (since $n \neq 3$ ) and centralizes a unique non-zero vector $b=\varphi_{B}^{-1} \varphi_{A}(a)$ in $B$. Since $\tau$ commutes with $K$, the claim follows from (e). Premultiplying $\tau$ by $\tau_{0}$, if necessary we can assume that $\tau$ normalizes both $A$ and $B$. Since they are absolutely irreducible $K$-modules, $\tau$ must be the identity and (iv) follows.

LEMMA 6.2. If $n=3$ then

(i) $G(x, y)$ contains two classes of $L_{2}(2) \cong S_{3}$-complements to $Q$, permuted by Out $G(x, y)$; 
(ii) $C_{\text {Aut } G(x, y)}(K) \cong S_{3}$ acts faithfully on $\{A Z, B Z, C Z\}$;

(iii) Out $G(x, y) \cong S_{3} \times 2$.

Proof. In the considered case $Q$ is extraspecial of order $2^{5}$ and plus type with $T=O_{3}(K)$ acting fixed-point freely on $Q / Z$. Since $T$ is a Sylow 3 -subgroup of $G(x, y)$ and $N_{G(x, y)}(T)=K \times Z$, (i) follows. If $K_{Z}$ is the second complement to $Z$ in $K \times Z$ then an automorphism $\lambda$ of $G(x, y)$ which maps $K$ onto $K_{Z}$ can be chosen to be an involution centralizing $O_{2,3}(G(x, y))=O^{2}(G(x, y))=Q T$. Since $T$ acts fixed-point freely on $Q / Z$, the normalizer of $K$ in Aut $Q \cong 2^{4}:\left(S_{3} 2 S_{2}\right)$ is isomorphic to $S_{3}$ which gives (ii) in view of Lemma 6.1 (d). Finally (iii) is immediate from (i) and (ii).

LEMMA 6.3. If $n=4$ then

(i) $G(x, y)$ contains six classes of $L_{3}(2)$-complements to $Q$;

(ii) $\left|C_{\text {Aut } G(x, y)}(K)\right|=2$;

(iii) Out $G(x, y)$ acts transitively on the set of $L_{3}(2)$-complements to $Q$ in $G(x, y)$;

(iv) $\operatorname{Out} G(x, y) \cong S_{3} \times 2$.

Proof. Consider first the quotient $\bar{Q}=Q / Z$. As a module for $\bar{K}=$ $K Z / Z \cong L_{3}(2)$ it is isomorphic to the direct sum of two copies of $W^{*}$. For $E \in\{A, B, C\}$ let $\bar{K}^{E}$ be the image of $\bar{K}$ under an outer automorphism of $(E Z / Z) \bar{K} \cong 2^{3}: L_{3}(2)$. Since $C$ is indecomposable as $K$-module, the preimage of $\bar{K}^{C}$ in $G(x, y)$ does not split over $Z$ by Lemma 3.11. On the other hand, the preimages of $\bar{K}^{A}$ and $\bar{K}^{B}$ are semidirect products of $Z$ with the images $K^{A}$ and $K^{B}$ of $K$ under outer automorphisms of $A K$ and $B K$, respectively. Thus, modulo $Z$, the group $G(x, y)$ contains three classes of $L_{3}(2)$-complements. Now for $S \in\left\{K, K^{A}, K^{B}\right\}$ let $S_{Z}$ be the image of $S$ under an outer automorphism of $Z S \cong 2^{3}: L_{3}(2)$. Then

$$
\mathcal{R}=\left\{K, K_{Z}, K^{A}, K_{Z}^{A}, K^{B}, K_{Z}^{B}\right\}
$$

is the complete set of representatives of the classes of $L_{3}(2)$-complements and (i) follows.

Let $S \in \mathcal{R}$. Since $Z$ is the centre of $Q$, whenever $s \in S$ and $s^{\prime}=s Z \cap S^{Z}$, we have $q^{s}=q^{s^{\prime}}$ for every $q \in Q$. Therefore a mapping $\mu$ which commutes with $Q$ and maps $s$ onto $s^{\prime}$ for every $s \in S$, is an automorphism of $G(x, y)$ 
which maps $S$ onto $S^{Z}$. We claim that $\mu$ acts fixed-point freely on the classes of complements. Indeed, we can choose the complements in $\mathcal{R}$ so that all the six share a subgroup $F \cong F_{7}^{3}$. Let $T$ be a Sylow 3 -subgroup in $F$. Then for $S \in \mathcal{R}$ the normalizer of $T$ in $S$ is isomorphic to $S_{3}$ and together with $F$ they generate $S$. It is clear from the structure of $G(x, y)$ that $N:=N_{G(x, y)}(T) / T$ is elementary abelian of order $2^{4} ; C:=C_{G(x, y)}(T) \leq Q$ and $C / T$ is a hyperplane in $N$. Since $\mu$ centralizes $C$ it induces on $N$ a transvection with axis $C / T$, hence the claim follows.

It is immediate from the proof of Proposition 6.1 that $\tau_{0}$ is the only non-identity automorphism of $G(x, y)$ which centralizes $K$, hence (ii) is established. It is clear that $\tau_{0}$ maps $\left\{K_{A}, K_{A}^{Z}\right\}$ onto $\left\{K_{B}, K_{B}^{Z}\right\}$.

Since $H^{1}\left(L_{4}(2), U^{[2]}\right)$ is non-trivial, there is an automorphism $\sigma_{0}$ of $G(x)$ which permutes two classes of $L_{4}(2)$-complements to $G_{1}(x)$. Let us discuss how the restriction $\sigma$ of $\sigma_{0}$ to $G(x, y)$ acts on the classes of $L_{3}(2)$ complements. By Lemma 3.14 we can assume that $L^{\sigma_{0}} \cap L \cong A_{7}$. Then $A^{\sigma_{0}} \cap A=1$ but $\sigma_{0}$ centralizes a complement to $A$ in $A K$. Since the complement, which is centralized is not a Levi complement, we can assume that $\sigma$ centralizes $K_{A}$. Since $\sigma$ normalizes $B Z=G_{1}(x)$ and does not normalize $A Z$ it must map the latter onto $C=C Z$. This shows that $\sigma$ maps $\left\{K, K^{Z}\right\}$ onto $\left\{K_{B}, K_{B}^{Z}\right\}$. Thus $\left\langle\mu, \tau_{0}, \sigma\right\rangle$ permutes transitively the classes of complements and (iii) is proved.

By the above discussion Out $G(x, y)$ induces $S_{3}$ on the set of pairs of classes of $L_{3}(2)$-complements with representatives $\left\{S, S^{Z}\right\}$, the kernel is generated by the image of $\mu$ and the image generates a direct factor. Hence we obtain (iv). $2^{2}$.

Lemma 6.4. If $n=5$ then Out $G(x, y)$ is elementary abelian of order

Proof. In terms of Proposition 6.1 in the considered situation $H^{1}(K, X)$ is trivial for $X=A, B$ and 1-dimensional for $X=Z$ (cf. Lemma 3.2). Thus there are two classes of complements to $Q$ in $G(x, y)$ with representatives $K$ and $K_{Z}$ where the latter is the image of $K$ under an automorphism $\nu_{0}$ of $Z K \cong 2^{6}: L_{4}(2)$, which acts trivially on both $Z$ and $Z K / Z$. Since $Z$ is the centre of $Q, K$ and $K_{Z}$ have the same image in the automorphism group of $Q$. Hence $\nu_{0}$ extends to an automorphism $\nu$ of $G(x, y)$. By our choice $\nu$ commutes with both $Q$ and $G(x, y) / Q$, which immediately shows that $\nu$ is an involutive automorphism and the result follows. 
As above let $\tau_{0}$ denote the automorphism of $G(x, y)$ as in (ii) in Proposition 6.1. For $n=3,4$ and 5 let $\tau_{1}$ be the product of $\tau_{0}$ with an automorphism which commutes with both $Q$ and $G(x, y) / Q$ and permutes the classes of complements to $Z$ in $Z K$ (the latter automorphism is denoted by $\lambda, \mu$ and $\nu$ in Lemmas 6.2, 6.3 and 6.4, respectively).

LEMMA 6.5. The following assertions hold:

(i) $C_{G(x, y)}\left(\tau_{0}\right) \cong 2^{n(n-1) / 2} \cdot L_{n-1}(2)$;

(ii) $C_{G(x, y)}\left(\tau_{1}\right)$ is isomorphic to

(a) $2^{3}: 3$ for $n=3$;

(b) $2^{3+3}: F_{7}^{3}$ for $n=4$ and

(c) $2^{6+4}: A_{7}$ or $2^{6+4}:\left(S_{5} \times S_{3}\right)^{+}$for $n=5$.

Proof. (i) is by the definition; for (ii) see the first paragraph in Subsection 3.1 and Lemma 3.14.

Lemma 6.6. Let $\mathcal{A}=\{G(x), G\{x, y\}\}$ be the amalgam associated with a locally projective action which corresponds to case (ii) in Proposition 6.1. Then one of the following holds:

(i) there is $t_{0} \in G\{x, y\}$ which induces on $G(x, y)$ the automorphism $\tau_{0}$, furthermore either

(a) $t_{0}^{2}=1$ and $\mathcal{A} \cong \mathcal{A}\left(O_{2 n}^{+}(2), D^{+}(2 n, 2)\right)$, or

(b) $n=3, t_{0}^{2}$ is the unique non-identity element in $Z$ and $\mathcal{A} \cong \mathcal{A}_{3}^{(1)}$;

(ii) $n=3,4$ or 5 and there is $t_{1} \in G\{x, y\}$ which induces the automorphism $\tau_{1}$ on $G(x, y)$, furthermore either

(c) $t_{1}^{2}=1$ and $\mathcal{A}$ is isomorphic to $\mathcal{A}_{3}^{(2)} \cong \mathcal{A}\left(M_{22}, \Gamma\left(\mathcal{G}\left(M_{22}\right)\right), \mathcal{A}_{4}^{(2)}\right.$ or $\mathcal{A}_{5}^{(1)}$, respectively, or

(d) $n=3, t_{1}^{2}$ is the unique non-identity element in $Z$ and $\mathcal{A} \cong \mathcal{A}_{3}^{(3)}$.

Proof. By Lemmas 6.1 (iv), 6.2, 6.3, 6.4 and in view of Lemmas 4.2 and 4.3 we have the possibilities (i) and (ii) (notice that for $n=3,4$ by Lemma 3.2 the group Out $G(x)$ is of order 2). For instance in the case $n=4$ the group Out $G(x, y)$, isomorphic to $S_{3} \times 2$ by Lemma 6.4 contains 7 involutions. Three of these involutions normalize $B Z=G_{1}(x)$ (which is 
already normal in $G(x)$ ). The remaining four involutions are contained in two cosets of the image in Out $G(x, y)$ of

$$
N_{\text {Aut } G(x)}(G(x, y))=\left\langle\operatorname{Inn} G(x), \sigma_{0}\right\rangle
$$

Finally, the images of $\tau_{0}$ and $\tau_{1}$ represent different cosets.

Since $C_{G(x, y)}\left(\tau_{i}\right) \leq C_{G(x, y)}\left(t_{i}\right)$, by Lemmas 6.1 and 6.5 the square of $t_{i}$ $(i=0$ or 1$)$ must be in the centre of $G(x, y)$, which is trivial unless $n=3$, in which case it is of order 2 .

Let $\Gamma$ be the graph of valency 31 on which $G \cong J_{4}$ acts 2 -arc-transitively with vertex stabilizer isomorphic to $2^{10}: L_{5}(2)$ (cf. [Iv87] and [SW88]). Then the amalgam $\{G(x), G\{x, y\}\}$ is isomorphic to $\mathcal{A}_{5}^{(1)}$. The graph $\Gamma$ contains geometric subgraphs of valency 3 (Petersen subgraphs) and 7 (derived graphs of the $P$-geometry $\mathcal{G}\left(M_{22}\right)$ ), but there are no geometric subgraphs of valency 15 and this explains the information given in Table 1.

A completion of $\mathcal{A}_{4}^{(2)}$ in $A_{64}$ that is constrained at level 2 was constructed in [IP04].

By Corollary 2.3 (i) neither $\mathcal{A}_{3}^{(1)}$ nor $\mathcal{A}_{3}^{(3)}$ possesses a constrained completion. One can observe that Aut $M_{22}$ is a (non-constrained) completion of the amalgam $\mathcal{A}_{3}^{(3)}$.

§7. $G_{3}(x)=1$

In this section we consider the case (iii) in Proposition 4.1. Thus $G(x)$ is the direct product of $G_{2}(x)=Z(G(x))$, which is of order 2 and the commutator subgroup of $G(x)$ (we denote this commutator subgroup by $\left.G(x)^{*}\right)$. In turn $G(x)^{*}$ is isomorphic to the semidirect product of $L \cong$ $G(x) / G_{1}(x) \cong L_{3}(2)$ and $G_{1}(x) / G_{2}(x)$, the latter being the natural module for $L$. Thus

$$
G(x) \cong G(x)^{*} \times G_{2}(x) \cong 2^{3}: L_{3}(2) \times 2
$$

LEMma 7.1. In the considered situation Out $G(x, y) \cong D_{8} \times S_{3}$.

Proof. Let $G(x, y)^{*}=G(x)^{*} \cap G(x, y)$. Then $G(x, y)^{*} \cong 2_{+}^{1+4}: S_{3}$ is isomorphic to the subgroup $G(x, y)$ in Lemma 6.1 when $n=3$. Hence Out $G(x, y)^{*} \cong S_{3} \times 2$ by Lemma 6.2 (iii). The whole of $G(x, y)$ is of course the direct product of $G(x, y)^{*}$ and $G_{2}(x)$, which is of order 2 .

Let $Z=Z(G(x, y)), Q=O_{2}(G(x, y))$ and $Z^{(0)}=Q^{\prime}$. Then by the above paragraph $Z=Z\left(G(x, y)^{*}\right) \times G_{2}(x)$ is elementary abelian of order 4 ; 
$Q=O_{2}\left(G(x, y)^{*}\right) \times G_{2}(x) \cong 2_{+}^{1+4} \times 2$ and $Z^{(0)}=Z\left(G(x, y)^{*}\right)$. With $Z^{(0)}$ as above let $\left\{Z^{(0)}, Z^{(1)}, Z^{(2)}\right\}$ be the set of subgroups of order 2 in $Z$. Let $H=O^{2}(G(x, y))$. Then $H \cong 2_{+}^{1+4} .3$ and $G(x, y) / H$ is elementary abelian of order 4 .

Thus there are exactly four ways to present $G(x, y)$ as a direct product $A \times B$ where $A$ is of order 2 from the centre of $G(x, y)$ and $B \cong G(x, y)^{*}$. Namely, $A$ is either $Z^{(1)}$ or $Z^{(2)}$ and $B$ is the preimage in $G(x, y)$ of a subgroup of order 2 in $G(x, y) / H$ other than $Z^{(1)} H / H=Z^{(2)} H / H$. Since Out $G(x, y)^{*}$ is known to be of order 12 , we conclude that Out $G(x, y)$ is of order 48.

Let $T \in S l_{3}(G(x, y))$ and $M$ be the normalizer of $T$ in Aut $G(x, y)$. Then by Frattini argument we have

$$
\text { Aut } G(x, y)=\operatorname{Inn} G(x, y) M .
$$

Let $S=N_{G(x, y)}(T) / T$. Then $S$ is elementary abelian of order 8 and $S_{1}=Z T / T$ is a hyperplane in $S$. Hence there are exactly four complements to $Q$ in $G(x, y)$, containing $T$ and these complements are transitively permuted by $M$. Since Aut $G(x, y)$ induces an action of order 2 on $Z$, we conclude that $M$ induces on $S$ the group $D_{8}$. On the other hand, the image of Aut $G(x, y)$ in $\operatorname{Out}(G(x, y) / Z)$ is isomorphic to $S_{3}$. Let $\nu$ be an automorphism of $G(x, y)$ which centralizes $Z$ and a complement $R$ to $O_{2}\left(G(x, y)^{*}\right)$ in $G(x, y)^{*}$ containing $T$ (in particular $\nu$ normalizes $\left.G(x, y)^{*}\right)$. We assume further that $\nu$ does not normalize $O_{2}\left(G(x)^{*}\right)$ (such an automorphism exists by the proof of Lemma 6.2). Then $\nu$ fixes the hyperplane $S_{1}$ in $S$ and the vector $R / T$, which is outside the hyperplane. Hence $\nu$ acts trivially on $S$ and at the same time it induces an outer automorphism of $G(x, y) / Z \cong 2^{4}: S_{3}$. Hence the result.

Lemma 7.2. Suppose we are in case (iii) of Proposition 4.1. Then $\mathcal{A}$ is isomorphic either to $\mathcal{A}_{3}^{(4)}$ or $\mathcal{A}_{3}^{(5)}$.

Proof. We follow notation introduced in the proof of Lemma 7.1. Let $t \in G\{x, y\} \backslash G(x, y), \tau \in$ Aut $G(x, y)$ be automorphism of $G(x, y)$ induced by $t$ and $\bar{\tau}$ be the image of $\tau$ on Out $G(x, y)$. Then $t^{2} \in G(x, y)$ and hence $\bar{\tau}^{2}=1$. In addition $t$ does not normalize neither $G_{2}(x)$ nor $O_{2}\left(G(x)^{*}\right)$. Hence $\bar{\tau}$ is an involution and by Lemma 7.1 (in view of Lemma 4.3) we have two possibilities for $\bar{\tau}$, which we denote by $\bar{\tau}_{1}$ and $\bar{\tau}_{2}$. Without loss of generality we assume that $t$ normalizes $T$ and then it induces a transposition 
on the set $S \backslash S_{1}$. In particular $t$ commutes with a complement $R \cong S_{3}$ to $\mathrm{O}_{2}(G(x, y))$ in $G(x, y)$. This shows that

$$
t^{2} \in Z_{0}=C_{G(x, y)}(R) \cap C_{G(x, y)}(t) .
$$

We claim that $Z t$ contains an involution. In fact, let $z_{i}$ denote the unique non-identity element of $Z_{i}, i=0,1,2$. Then $t^{-1} z_{1} t=z_{2}$ and $z_{0} z_{1} z_{2}=1$. Suppose that $t^{2}=z_{0}$. Then $\left(t z_{1}\right)^{2}=t^{2} t^{-1} z_{1} t z_{1}=t^{2} z_{2} z_{1}=1$ and the claim follows. By Lemmas 4.2 and 4.3 the isomorphism type of $\mathcal{A}$ is uniquely determined by the choice between $\bar{\tau}_{1}$ and $\bar{\tau}_{2}$.

\section{$\S 8 . \mathrm{Co}_{2}$-type amalgam}

In this section we deal with case (iv) of Proposition 4.1. Therefore we assume that $n=4, G_{1}(x) / G_{2}(x) \cong U^{[2]}, G_{2}(x) / G_{3}(x) \cong U$ and $\left|G_{3}(x)\right|=2$ (where $U$ is the natural module for $L=G(x) / G_{1}(x) \cong L_{4}(2)$ ). Some further information on the structure of $G(x)$ was announced in [Tr00], while in [Tr03b] the group $G(x)$ was specified up to isomorphism. Unfortunately the terms in which the specification was made is not very convenient for our purpose, so we will not use it and only apply the above information on $G(x)$. We will prove eventually (cf. Proposition 8.23) that the amalgam $\mathcal{A}=\{G(x), G\{x, y\}\}$ (and particularly the vertex stabilizer $G(x)$ ) is unique up to isomorphism and corresponds to the action of $\mathrm{Co}_{2}$ on the derived graph of its $P$-geometry.

\section{1. $G(x) / Z(G(x))$}

We first need some technical results.

LEMMA 8.1. The following assertions hold:

(i) there is a unique indecomposable extension $V$ of a natural module $U$ for $L_{4}(2)$ by its exterior square $U^{[2]}$;

(ii) $H^{1}\left(L_{4}(2), V\right)$ is trivial;

(iii) $H^{1}\left(L_{4}(2), V^{*}\right)$ is 1-dimensional.

Proof. The assertion (i) follows from Table I in [B78], since by a standard result the number of extensions under consideration equals to the number of non-identity elements in

$$
H^{1}\left(L_{4}(2), H_{G o m(2)}\left(U, U^{[2]}\right)\right) .
$$

The extension $V$ is known as the module of quadratic forms on $U$. 
Let $\mathcal{P}$ be the $G F(2)$-permutation module for $L_{4}(2)$ acting on the 1 subspaces in $U$. It is well known (cf. Sections 3.2 and 3.3 in [IS02]) that $\mathcal{P}=$ $\mathcal{P}_{e} \oplus \mathcal{P}_{c}$, where $\mathcal{P}_{c}$ is 1 -dimensional and $\mathcal{P}_{e}$ possesses the unique composition series

$$
\mathcal{P}_{e}=\mathcal{X}(1)>\mathcal{X}(2)>\mathcal{X}(3)>\mathcal{X}(4)=0,
$$

where $\mathcal{X}(i) / \mathcal{X}(i+1) \cong U^{[i]}$ for $1 \leq i \leq 3$. Similarly, if $\mathcal{H}$ is the $G F(2)$ permutation module of $L_{4}(2)$ acting on the 3-subspaces (hyperplanes) in $U$, then $\mathcal{H}=\mathcal{H}_{c} \oplus \mathcal{H}_{e}$, where $\mathcal{H}_{c}$ is 1-dimensional and

$$
\mathcal{H}_{e}=\mathcal{Y}(1)>\mathcal{Y}(2)>\mathcal{Y}(3)>\mathcal{Y}(4)=0
$$

is the unique composition series for $\mathcal{H}_{e}$, where $\mathcal{Y}(i) / \mathcal{Y}(i+1) \cong U^{[4-i]} \cong$ $\left(U^{[i]}\right)^{*}$. By $(\mathrm{i})$ this means that $V \cong \mathcal{Y}(2)$ and $V^{*} \cong \mathcal{X}(1) / \mathcal{X}(3)$.

By Lemma 3.2 each $H^{1}\left(L_{4}(2), V\right)$ and $H^{1}\left(L_{4}(2), V^{*}\right)$ is either trivial or 1-dimensional. Suppose that $H^{1}\left(L_{4}(2), V\right)$ is non-trivial. Then, according to a general principle there exists an indecomposable extension $\widetilde{V^{*}}$ of a 1 dimensional trivial module by $V^{*}$. By the previous paragraph $L_{4}(2)$ acting on $V^{*}$ contains an orbit $\Omega$ of length 15 indexed by the hyperplanes in $U$ and $V^{*}$ is generated by the vectors in $\Omega$. Thus the stabiliser $S$ in $L_{4}(2)$ of a vector from $\Omega$ is isomorphic to $2^{3}: L_{3}(2)$. Let $\widetilde{\Omega}$ be the preimage of $\Omega$ in $\widetilde{V^{*}}$. Since $S$ does not contain subgroups of index $2, \widetilde{\Omega}$ is the union of two $L_{4}(2)$-orbits of length 15 each. Since $\widetilde{V^{*}}$ is an indecomposable extension of $V^{*}$, each of the two orbits generates $\widetilde{V^{*}}$, in particular $\widetilde{V^{*}}$ is a quotient of $\mathcal{H}$. By the above paragraph the latter contains a unique 1-dimensional composition factor which is a direct summand. Thus the module $\widetilde{V^{*}}$ with the required properties does not exist, which proves (ii).

In order to prove (iii) recall that $U^{[2]}$ is isomorphic to the heart of the $G F(2)$-permutation module $\mathcal{E}$ of $L_{4}(2) \cong A_{8}$ acting naturally on 8 points. Furthermore $\mathcal{E}$ possesses the unique composition series

$$
\mathcal{E}>\mathcal{E}_{e}>\mathcal{E}_{c}>0
$$

where both $\mathcal{E} / \mathcal{E}_{e}$ and $\mathcal{E}_{c}$ are 1 -dimensional and $\mathcal{E}_{e} / \mathcal{E}_{c} \cong U^{[2]}$. Now considering the sub-direct product of $V$ and $\mathcal{E}_{e}$ with respect to $U^{[2]}$, we obtain an indecomposable extension of the 1-dimensional module by $V$. Applying the general principle once again, we see that $H^{1}\left(L_{4}(2), V^{*}\right)$ is 1-dimensional.

We follow notation introduced in Subsection 3.2 for $L \cong L_{4}(2)$, its subgroups and modules. In particular $D$ denotes the semidirect product of 
$U^{[2]}$ and $L$ with respect to the natural action. Furthermore, we identify $U$ with the corresponding $L$-submodule in $V$ (as in Lemma 8.1 (i)) and $U^{[2]}$ with $V / U$.

Lemma 8.2. Let $X$ be a group such that $X / O_{2}(X) \cong L \cong L_{4}(2)$, $\mathrm{O}_{2}(X)$ is elementary abelian isomorphic to $V$ as an L-module. Let $N_{1}$ and $N_{2}$ be the preimages in $X$ of representatives of the two classes of complements to $\mathrm{O}_{2}(D)$ in $X / U \cong D$. Then, up to renumbering, $N_{1} \cong U: L$ (semidirect product), while $N_{2} \cong 2^{4} \cdot L_{4}(2)$ (the non-split extension). In particular $H^{2}\left(L_{4}(2), V\right)$ is trivial.

Proof. Let $\widehat{N}_{1} \cong 2^{4} \cdot L_{4}(2)$ be the unique non-split extension of $U$ by $L$. Let $\widehat{X}$ be the semidirect product of $V$ and $\widehat{N}_{1}$ with $O_{2}\left(\widehat{N}_{1}\right)$ acting trivially and $\widehat{N}_{1} / O_{2}\left(\widehat{N}_{1}\right)$ acting in the natural way. Then, arguing as in the proof of Lemma 3.11 and applying Lemmas 3.3 and 8.1 (i), we observe that $\widehat{X}$ is the largest group subject to the following properties: (1) $\widehat{X} / O_{2}(\widehat{X}) \cong L_{4}(2)$; (2) $\mathrm{O}_{2}(\widehat{X})$ is elementary abelian; (3) $\widehat{X}$ possesses a homomorphism onto $D$ whose kernel $J$ is a direct sum of modules isomorphic to $U$; (4) if $T$ is a subgroup in $\widehat{X}$ such that $\widehat{X}=T J$, then $T=\widehat{X}$. Let $\sigma$ be the automorphism of $D$ which permutes the classes of $L_{4}(2)$-complements and commutes with $D / O_{2}(D)$. Then by the maximality property and since $\sigma$ preserves the natural module $U, \sigma$ extends to an automorphism of $\widehat{X}$. Let $\widehat{N}_{2}$ be the image of $\widehat{N}_{1}$ under this extension. Since $H^{1}\left(L_{4}(2), V\right)$ is trivial by Lemma 8.1 (ii) we observe that $O_{2}\left(\widehat{N}_{1}\right) \neq O_{2}\left(\widehat{N}_{2}\right)$, that $J=O_{2}\left(\widehat{N}_{1}\right) \oplus O_{2}\left(\widehat{N}_{2}\right)$ and that the group $X$ in the hypothesis of the lemma is the quotient of $\widehat{X}$ over $O_{2}\left(\widehat{N}_{i}\right)$ for $i=1$ or 2 . Now the last sentence in the claim is immediate in view of Lemma 3.3 (which tells us that $L_{4}(2)$ always splits over the exterior square of its natural module).

The description in the proof of Lemma 8.1 of the $L$-module $V$ as a submodule of the $G F(2)$-permutation module on the hyperplanes in $U$ gives the following (compare the characterization of the exterior powers of $U$ in Lemma 3.1.3 in [IS02]).

Lemma 8.3. There is a injection $d$ of the set of 2-subspaces in $U$ into $V^{\#}$. If $s_{1}, s_{2}, s_{3}$ are 2-subspaces such that $\left\langle s_{1}, s_{2}, s_{3}\right\rangle$ is a hyperplane and $s_{1} \cap s_{2} \cap s_{3}$ is a 1-subspace generated by $u \in U^{\#}$, then

$$
d\left(s_{1}\right) d\left(s_{2}\right) d\left(s_{3}\right)=u .
$$


Notice that if we consider $U$ as a submodule in $V$, so that $V / U \cong U^{[2]}$ then the mapping which sends a 2-subspace $s$ of $U$ onto $d(s) U$ coincides with the mapping $d_{2}$ defined before Lemma 3.1. We continue to use notation from Subsection 3.2. Recall that $U=\langle w\rangle \oplus W$ is a direct sum decomposition of $U$, $P_{1}$ and $P_{2}$ are the stabilizers in $L$ of $\langle w\rangle$ and $W$, respectively and $K_{0}$ (called the Levi complement) is the stabilizer of this direct sum decomposition. Let

$$
V(w)=\langle d(s) \mid s \sim w\rangle \text { and } V(W)=\langle d(s) \mid s \sim W\rangle
$$

be subspaces in $V$ (compare the definition before Lemma 3.16).

LEMMA 8.4. The following assertions hold:

(i) $V(w)=C_{V}\left(O_{2}\left(P_{1}\right)\right)$;

(ii) $V(W)=C_{V}\left(O_{2}\left(P_{2}\right)\right)$;

(iii) $V(w)$, as a module for $P_{1} / O_{2}\left(P_{1}\right) \cong L_{3}(2)$, is the direct sum of $\langle w\rangle$ and a 3-dimensional submodule isomorphic to $U /\langle w\rangle$;

(iv) $V(W)$, as a module for $P_{2} / O_{2}\left(P_{2}\right) \cong L_{3}(2)$, is isomorphic to the even half of the $G F(2)$-permutation module on the 2-subspaces in $W$;

(v) the Levi complement $K_{0}$ is the stabilizer in $L$ of the direct sum decomposition $V=V(w) \oplus V(W)$.

Proof. It is immediate from the definition that $V(w) U / U=U^{[2]}(w)$ and $V(W) U / U=U^{[2]}(W)$. Furthermore, it is easy to deduce from the relations in Lemma 8.3 that $V(w) \cap U=\langle w\rangle$ and $V(W) \cap U=W$. Therefore, particularly, $\operatorname{dim} V(w)=4, \operatorname{dim} V(W)=6$ and $V(w) \cap V(W)=0$. Since $O_{2}\left(P_{1}\right)$ centralizes every generator of $V(w)$, the latter is contained in $C_{V}\left(O_{2}\left(P_{1}\right)\right)$. On the other hand $C_{U^{[2]}}\left(O_{2}\left(P_{1}\right)\right)=U^{[2]}(w)$ by Lemma 3.16 (i) and clearly $C_{U}\left(O_{2}\left(P_{1}\right)\right)=\langle w\rangle$, hence (i) follows. Similar argument establishes (ii).

By the definition $V(w)$ is a quotient of the $G F(2)$-permutation module of $P_{1}$ acting of the 1-subspaces in $U /\langle w\rangle$ while $V(W)$ is a quotient of the $G F(2)$-permutation module of $P_{2}$ acting on the 2-subspaces in $W$. Now (iii) and (iv) are immediate in view of Lemma 3.6 (i) and the dimensions of $V(w)$ and $V(W)$. Notice that the complement to $\langle w\rangle$ in $V(w)$ is generated by the elements $d(s) w$ taken for all the 2 -subspaces in $U$ containing $w$. Since $K_{0}$ is the stabilizer in $L$ of the direct sum decomposition $U=\langle w\rangle \oplus W$ assertion (v) follows from Lemma 3.16 (iii). 
Lemma 8.5. Suppose that the case (iv) in Proposition 4.1 holds. Then the following assertions hold:

(i) $G_{2}(x)=Z\left(G_{1}(x)\right)$, in particular $G_{1}(x)$ is non-abelian;

(ii) $G_{2}(x)$ is an elementary abelian 2-group and as an L-module it is isomorphic to the direct sum of $G_{3}(x)=Z(G(x))$ and $U \cong G_{2}(x) / G_{3}(x)$;

(iii) $G(x) / G_{3}(x)=V: \bar{M}$, where $\bar{M} \cong L \cong L_{4}(2)$ and $V=G_{1}(x) / G_{3}(x)$ is the indecomposable L-module as in Lemma 8.1 (i);

(iv) the group $G(x) / G_{3}(x)$ is complete.

Proof. Let $W=G_{2}(x)$. Since the two chief factors of $G(x)$ in $W$ are 4-dimensional and 1-dimensional, $W$ is elementary abelian. Considering it as a $G(x)$-module, we further observe that $G_{1}(x)$ acts trivially on $W$, since $G_{1}(x) / W$ is 6-dimensional irreducible. Thus, $G(x)$ induces on $W$ the group $L_{4}(2)$. It now follows from Lemma 3.2 that $W$ is semi-simple. Let $E_{2}$ be the 4-dimensional submodule in $W$. Clearly, $E_{2}$ is normal in $G(x)$.

Next, let $Z_{x}=G_{3}(x)$ and consider the group $V=G_{1}(x) / Z_{x}$. We claim that $V$ is elementary abelian. Indeed, let $V_{0}$ be the image of $W$ in $V$. The mapping $v V_{0} \mapsto v^{2}$ is a well-defined mapping from $V / V_{0}$ to $V_{0}$, and this mapping clearly commutes with the action of $G(x)$. By Lemma 3.15 the latter group has two orbits (of lengths 28 and 35) on the nonzero vectors of $V / V_{0}$, corresponding to the stabilizers $S_{6}$ and $2^{4}:\left(S_{3} \times S_{3}\right)$ (stabilizer of a 2-subspace from the natural module). None of these two stabilizers fixes a vector in $V_{0}$. Hence the above mapping sends every vector from $V_{0} / V$ to zero. This means that $V$ is elementary abelian. In particular, the commutator subgroup of $G_{1}(x)$ is contained in $Z_{x}$.

We now argue that $G_{1}(x)$ is non-abelian, which implies that $\left[G_{1}(x)\right.$, $\left.G_{1}(x)\right]=Z_{x}$. If $G_{1}(x)$ is abelian then also $G_{1}(y)$ is abelian. Observe that $G_{1}(y) \leq G(x)$ and $G_{1}(y) \not G_{1}(x)$. Therefore, $G_{1}(y)$ acts non-trivially on $W$, which is a contradiction since $W \leq G_{1}(y)$. Thus, $G_{1}(x)$ is non-abelian.

The mapping from $G_{1}(x) / W$ to $Z_{x}$ defined by $g W \mapsto g^{2}$ is a nontrivial (and hence non-degenerate) $G(x)$-invariant quadratic form. Since the orbits of $G(x)$ on $\left(G_{1}(x) / W\right)^{\#}$ are of lengths 28 and 35 , this form is of plus type. Setting $E_{1}$ to be the full preimage in $G_{1}(x)$ of an arbitrary complement to $V_{0}$ in $V$, we see that $E_{1}$ is extraspecial of plus type with centre $Z_{x}$, completing the proof of (i) and (ii).

We next show that $V$ is indecomposable as a $G(x)$-module. (Clearly, $G_{1}(x)$ acts trivially on $V$ and hence $G(x)$ induces on $V$ the group $\left.L_{4}(2)\right)$. Let $X=G\{x, y\}, R=O_{2}(G(x, y))$, and let $t \in X$ be an element mapping 
to the involution in the centre of $X / R \cong 2 \times L_{3}(2)$. If $V$ is semi-simple then $E_{1}$ can be chosen to be normal in $G(x)$. In that case it is also normal in $R$. Also $E_{1}^{t}$ is normal in $R$. Since $Z_{y}=Z_{x}^{t} \neq Z_{x}$ and $Z_{x}^{t}=G_{3}(y) \leq W$, we get that $T=E_{1} \cap E_{1}^{t}$ is a normal subgroup of $E_{1}^{t}$ not containing $Z_{x}^{t}$. This is impossible, because $E_{1}^{t}$ is extraspecial. Therefore, $T=1$, implying that $R=$ $E_{1} \times E_{1}^{t}$. Consequently, $[R, R]$ is of order four. This is a contradiction, since $R$ acting on $V / V_{0}$ already produces a 3-dimensional commutator subspace.

We have shown that $V$ is an indecomposable extension of a 4-dimensional $V_{0}$ by 6-dimensional module $V / V_{0}$. Such an extension is unique by Lemma 8.1 (i), hence it is as claimed in (iii). By Lemma 8.2, $G(x) / Z_{x}$ splits over $V$, completing the proof of (iii).

Let $\tau$ be an automorphism of $G(x) / Z_{x}$. In order to prove (iv) we have to show that $\tau$ is inner. By (iii) and Lemma 8.1 (ii) we can assume that $\tau$ normalizes a complement $\bar{M} \cong L_{4}(2)$ to $V=O_{2}\left(G(x) / Z_{x}\right)$. Suppose that $\tau$ induces an outer automorphism of $\bar{M}$. Then it maps the natural module $G_{2}(x) / Z_{x}$ onto its dual and the latter is not involved in $G(x) / Z_{x}$. Hence we can assume that $\tau$ centralizes $\bar{M}$. Since both $E_{1} / Z_{x}$ and $E_{2} Z_{x} / Z_{x}$ are absolutely irreducible $\bar{M}$-modules and not dual to each other, we conclude that $\tau$ centralizes $V$ which completes the proof.

Remark. The above lemma specifies $H:=G(x) / Z_{x}$ up to isomorphism, as the semidirect product of $V$ and $\bar{M} \cong L_{4}(2)$ with respect to the natural action.

To list all the possibilities for the structure of $G(x)$, we have so far, we need to compute the Schur multiplier of $H$.

LEMmA 8.6. The Schur multiplier of $H=G(x) / Z_{x}$ is elementary abelian of order eight.

Proof. Let $\widehat{H}$ be the covering group of $H$. Since $L$ does not preserve non-zero alternating forms on $U$ and $H^{1}\left(L_{4}(2), U^{*}\right)$ is trivial, arguing exactly as in the proof of Lemma 3.17, we show that the order of $Z(\widehat{H})$ is at most 8 . On the other hand, if $\widehat{D}$ be the covering group of $D \cong 2^{6}: L_{4}(2)$ as in Lemma 3.17. Then the subdirect product of $H$ and $\widehat{D}$ with respect to $D$ is a perfect central extension of $Z(\widehat{D})$ by $H$ which is the universal extension by the order reason.

Lemmas 8.5 and 8.6 determine the structure of $G(x)$ up to four possibilities. (Indeed, $G(x)$ is obtained from $\widehat{H}$ be factoring over one of the four 
central subgroups of order four not containing the commutator subgroup of order 2 of $\mathrm{O}_{2}(\widehat{H})$.) In two of those groups the preimage $M$ of the complement $\bar{M}$ is isomorphic to $2 \times L_{4}(2)$ and in the other two it is isomorphic to the full covering group of $2 \cdot L_{4}(2)$ of $L_{4}(2)$. Notice that these four quotients are pairwise non-isomorphic by Lemma 8.5 (iv). The information on $G(x)$ given in Lemma 8.5 (i), (ii), (iii) and the fact that $M \cong 2 \cdot L_{4}(2)$ was announced in [Tr00]. As we have just seen this still leaves two possibilities for the isomorphism type of $G(x)$ (as we have already mentioned, the structure of $G(x)$ was specified up to isomorphism in [Tr03b]).

\section{2. $G(x, y) / Z(G(x, y))$}

We now turn our attention to the group $G\{x, y\}$. Let $t \in G\{x, y\}$ be as above. That is, $t$ maps onto the involution in the centre of $G\{x, y\} / O_{2}(G(x$, $y)) \cong 2 \times L_{3}(2)$. Let $E=G_{3}(x) G_{3}(y)=Z_{x} Z_{x}^{t}, R=G_{2}(x) G_{2}(y)$ and $Q=G_{1}(x) G_{1}(y)$. Clearly, these subgroups are normal in $G\{x, y\}$. Set $A=R / E$ and $B=Q / R$.

Lemma 8.7. The groups $E, A$ and $B$ are elementary abelian 2-groups, and $\mathrm{O}_{2}(G(x, y))$ acts on these $G F(2)$-spaces trivially. Viewing them as modules for $G(x, y) / O_{2}(G(x, y)) \cong L_{3}(2)$, we have the following:

(i) $E=G_{3}(x) \oplus G_{3}(y)$ is a direct sum of two 1-dimensional modules permuted by $t$

(ii) $A=G_{2}(x) / E \oplus G_{2}(y) / E$ is a direct sum of two isomorphic irreducible 3-dimensional modules permuted by $t$;

(iii) $B=G_{1}(x) / R \oplus G_{1}(y) / R$ is also a direct sum of two isomorphic irreducible 3-dimensional modules permuted by $t$; and

(iv) $B$ is dual to $A$.

Furthermore, $Q=\mathrm{O}_{2}(G(x, y))$.

Proof. Since $O_{2}(G(x, y)) / G_{1}(x)$ is an irreducible module for $G(x, y) /$ $O_{2}(G(x, y)) \cong L_{3}(2)$ and since $G_{1}(x)$ is not normal in $G\{x, y\}$, we obtain that $Q=\mathrm{O}_{2}(G(x, y))$.

Part (i) holds because $G_{3}(x)=Z_{x}$ is in the centre of $G(x)$, but it is not normal in $G\{x, y\}$. Now observe that $E \leq G_{2}(x)$ and that $G_{2}(x) / E \cong 2^{3}$ is invariant under $G(x, y)$, which induces on it $L_{3}(2)$ acting irreducibly. This yields that $Q$ acts on $G_{2}(x) / E$ trivially. Now (ii) follows since again $G_{2}(x)$ cannot be invariant under $t$. Part (iii) can be proved similarly. Finally part (iv) follows because the two chief factors of $G(x, y)$ in $G_{1}(x) / G_{2}(x)$ are dual to each other. 
We will view the chief factors of $G(x, y)$ found in $A$ as the natural modules for $G(x, y) / Q \cong L_{3}(2)$, and the chief factors found in $B$ as the dual natural modules.

Choose a complement $\bar{M}$ to $V$ in $G(x) / Z_{x}$ (all such complements are conjugate by Lemma 8.1 (ii)), and let $M$ be the full preimage of $\bar{M}$ in $G(x)$. Then $M \cong 2 \times L_{4}(2)$ or $M \cong 2 \cdot L_{4}(2)$. Let $N=G(x, y) \cap M$. Then $\bar{N}=N / Z_{x} \cong 2^{3}: L_{3}(2)$ is the stabilizer in $\bar{M} \cong L_{4}(2)$ of the 1-dimensional subspace $E / Z_{x}$ in the natural module $U=G_{2}(x) / Z_{x}$. Choose a Levi complement $L_{3}(2)$ in the parabolic $\bar{N}$ and let $T$ be the commutator subgroup in the full preimage in $M$ of this Levi complement. By Lemma 3.13 we have the following.

LEMmA 8.8. The $G(x, y)$ splits over $Q=O_{2}(G(x, y))$, namely $T \cong$ $L_{3}(2) \cong G(x, y) / Q$.

We follow the notation introduced before Lemma 8.7 and study further the structure of $G(x, y)$. Recall that this subgroup is the preimage in $G(x)$ of the stabilizer in $L=G(x) / G_{1}(x)$ of the 1-subspace $\langle w\rangle=E / Z_{x}$ in the natural module $U=G_{2}(x) / G_{3}(x)$. Then $G(x, y) / G_{1}(x)$ coincides with the parabolic $P_{1} \cong 2^{3}: L_{3}(2)$ in $L=G(x) / G_{1}(x)$. By Lemma $8.8 G(x, y)$ splits over $Q=\mathrm{O}_{2}(G(x, y))$. We will classify the classes of $L_{3}(2)$-complements in $G(x, y)$ and show that $t$ must preserve one of those classes. Our nearest goal is to study the structure (the classes of $L_{3}(2)$-complements, the Schur multiplier and the automorphism group) of

$$
F:=G(x, y) / E \text {. }
$$

Notice that by Lemma 8.5 (iii) the group $F$ is determined uniquely up to isomorphism independently of the choice between the four possibilities for $G(x)$ we have so far (cf. the paragraph after the proof of Lemma 8.6).

Let $P=O_{2}(F)$, which is the image of $Q=\left\langle G_{1}(x), G_{1}(y)\right\rangle$ in $F$. Then in terms of Lemma $8.7 A$ is a normal subgroup of $F$ contained in $P$ and $B=P / A$. Put $A(x)=G_{2}(x) / E, A(y)=G_{2}(y) / E$ and let $A(e)$ be the third 3-dimensional $F / P$-submodule in $A$ (here $e$ symbolises the edge $\{x, y\}$ ). Similarly put $B(x)=G_{1}(x) / R, B(y)=G_{1}(y) / R$ and let $B(e)$ be the third 3-dimensional $F / P$-submodule in $B$. Thus for $\alpha \in\{x, y, e\} A(\alpha)$ is the natural module for $F / P \cong L_{3}(2)$ and $B(\alpha)$ is the dual natural module. It is clear that the automorphism of $F$ induced by $t \in G\{x, y\} \backslash G(x, y)$ swaps $A(x)$ with $A(y)$ and $B(x)$ with $B(y)$. Therefore it stabilizes $A(e)$ and $B(e)$. 
As in Lemma 8.8 let $T$ be commutator subgroup of the full preimage in $M$ of a Levi complement in the parabolic $\bar{N} \cong 2^{3}: L_{3}(2)$ (sometimes we will identify $T$ with its isomorphic image in $F$ ). We can assume that $T$ stabilizes the direct sum decomposition

$$
U=\langle w\rangle \oplus W
$$

where $\langle w\rangle=E / Z_{x}$ and $W$ is a hyperplane in $U=G_{2}(x) / Z_{x}$. Consider $V(w)$ and $V(W)$ defined before Lemma 8.4 as subgroups in $G_{1}(x) / Z_{x} \cong V$, let $Y(w)$ and $Y(W)$ be their respective images in $P \cong V /\langle w\rangle$ (we consider these images as $T$-modules).

LEMMA 8.9. The following assertions hold:

(i) $Y(W) \cong V(W), Y(W) \cap A=A(x)$ and $Y(W) A / A=B(x)$;

(ii) $Y(w)=A(\alpha)$ for $\alpha=y$ or $\alpha=e$;

Proof. This is immediate from Lemmas 8.4 and 8.7 .

Let $N$ be as in the paragraph before Lemma 8.8, and $I$ be the image in $F$ of $O_{2}(N)$ (notice that $I \cong O_{2}(N) / Z_{x} \cong 2^{3}$ ).

LEMma 8.10. The following assertions hold:

(i) $I A / A=B(\beta)$ for $\beta=y$ or $\beta=e$;

(ii) $I A$ is elementary abelian and as a T-module it is the direct sum $I \oplus$ $A(x) \oplus A(y)$.

Proof. Since $\bar{N}=N / Z_{x}$ is the stabilizer of $\langle w\rangle=E / Z_{x}$ in $\bar{M} \cong L_{4}(2)$, $\mathrm{O}_{2}(N) / Z_{x}$ is the group of transvections with centre $w$. Therefore $I$ and $W^{*}$ are isomorphic as $T$-modules and (i) follows. Furthermore, $I$ commutes with both $A(x)=U /\langle w\rangle$ and $Y(W)$. Hence (ii) follows from Lemmas 8.7 (ii) and 8.9 (ii).

For a 2-subspace $s$ in $W$ let $i(s)$ denote the transvection in $O_{2}(N) / Z_{x}$ with axis $\langle w, s\rangle$ and centre $w$ (sometimes we will identify this transvection with its image in $I)$.

Lemma 8.11. Let $P^{\prime}$ be the commutator subgroup of $P=O_{2}(F)$. Then

(i) $P^{\prime}=A(e)$; 
(ii) $Y(w)=A(y)$;

(iii) $A$ is the centre of $P$.

Proof. By Lemma $8.7 B=P / A$ is elementary abelian and hence $P^{\prime} \leq$ $A$. By Lemmas 8.9 and $8.10 A$ is in the centre of $P$. Furthermore both $Y(W)$ and $I$ are abelian and $P=Y(W) I A$. Therefore $P^{\prime}$ is generated by the images in $F$ of the commutators $\left[d\left(s_{1}\right), i\left(s_{2}\right)\right]$ taken for all the pairs $s_{1}$, $s_{2}$ of 2 -subspaces in $W$. If $s_{1}=s_{2}$ then clearly the commutator is trivial. So let $s_{1} \neq s_{2}$ and $s_{1} \cap s_{2}=\langle u\rangle$, where $u \in W^{\#}$. Then $i\left(s_{2}\right) d\left(s_{1}\right) i\left(s_{2}\right)=d\left(s_{3}\right)$ where $s_{3}$ is the 2 -subspace in $U$ which is the image of $s_{1}$ under $i\left(s_{2}\right)$. Since $s_{3}$ contains $u$ there is a unique 2-subspace $s_{4}$ other than $s_{1}$ and $s_{3}$ contained in the hyperplane $\left\langle s_{1}, s_{3}\right\rangle$ and containing $u$. Then

$$
\begin{aligned}
{\left[d\left(s_{1}\right), i\left(s_{2}\right)\right] } & =i\left(s_{2}\right) d\left(s_{1}\right) i\left(s_{2}\right) d\left(s_{1}\right)=d\left(s_{3}\right) d\left(s_{1}\right) \\
& =d\left(s_{4}\right) d\left(s_{4}\right) d\left(s_{3}\right) d\left(s_{1}\right)=d\left(s_{4}\right) u
\end{aligned}
$$

where the latter equality is by Lemma 8.3. Since $s_{4}$ contains both $w$ and $u$, in terms of Lemma 8.9 and its proof, we have $\left[d\left(s_{1}\right), i\left(s_{2}\right)\right]=\chi(u) u$. This shows that $P^{\prime}$ is a $T$-invariant subgroup of order $2^{3}$ in $A$. By Lemma 8.9 we observe that $P^{\prime} \neq A(x)$ and clearly $P^{\prime} \neq Y(w)$. Since $P^{\prime}$ is characteristic in $F$, we conclude that $P^{\prime}=A(e)$ and hence $Y_{0}(w)=A(y)$, which gives (i), (ii) and (iii).

Let $J_{0}(W)$ denote the subgroup in $G(x) / Z_{x}$ generated by the elements $d(s) i(s)$ taken for all the seven 2-subspaces $s$ in $W$. We have seen within the proof of Lemma 8.11 that whenever $s_{1} \cap s_{2}=\langle u\rangle$, the equality

$$
\left[d\left(s_{1}\right), i\left(s_{2}\right)\right]=\chi(u) u
$$

holds and $\chi(u) u \in A(e) \leq A=Z(P)$. This enables us to check that the generators of $J_{0}(W)$ pairwise commute. Therefore $J_{0}(W)$ is elementary abelian and as a $T$-module it is a quotient of the permutation module on 2-subspaces in $W$. Let $\left\{s_{1}, s_{2}, s_{3}\right\}$ be the triple of such subspaces containing a given vector $u \in W^{\#}$. The the above commutator equality gives

$$
\prod_{j=1}^{3} d\left(s_{j}\right) i\left(s_{j}\right)=\chi(u)
$$

Since $\chi(u) \in A(y)$ by Lemmas 8.9 and 8.11 (ii), we obtain the following 
LEMMA 8.12. The following assertions hold:

(i) $J_{0}(W)$ (as a T-module) is isomorphic to the even half of the permutation module on the set of 2-subspaces in $W$;

(ii) $J_{0}(W)$ is isomorphic to its image $J(W)$ in $F ; J(W) \cap A=A(y)$, $J(W) A / A=B(y)$ and $I=B(e)$.

As an immediate consequence of Lemmas 8.9, 8.10 (ii) and 8.12 (iii) we obtain the following.

LEMMA 8.13. $I A / A=B(e)$.

\subsection{Some automorphisms of $G(x, y) / Z(G(x, y))$}

In order to deduce certain properties of the automorphism group of $F$ we need to know the classes of complements to $\mathrm{O}_{2}(F)$ in $F$.

Lemma 8.14. The group $F=G(x, y) / Z(G(x, y))$ contains exactly 8 classes of $L_{3}(2)$-complements to $P=\mathrm{O}_{2}(F)$.

Proof. Here we identify the subgroup $T \cong L_{3}(2)$ from Lemma 8.8 with its (isomorphic) image in $F$, so that $T$ is one of the $L_{3}(2)$-complements we are after. Consider first $\bar{F}=F / A$ which is the semidirect product of $\bar{T}$ and the direct sum of two copies of the dual natural module of $\bar{T}$. For $\alpha \in\{x, y, e\}$ the group $B(\alpha) \bar{T} \cong 2^{3}: L_{3}(2)$ contains two classes of $L_{3}(2)$ complements. Let $\bar{T}^{\alpha}$ denote the image of $\bar{T}$ under an outer automorphism of $B(\alpha) \bar{T}$. By Lemmas $8.9,8.12$ the preimages of $B(x)$ and $B(y)$ involve an indecomposable $T$-module (the even half of the permutation module of dimension 7), while the preimage of $B(e)$ is semi-simple. Hence (cf. Lemma 3.11) the preimages of $\bar{T}^{x}$ and $\bar{T}^{y}$ involve the non-split extension $2^{3} \cdot L_{3}(2)$, while the preimage of $\bar{T}^{e}$ splits over $A$ and we denote by $T^{e}$ the corresponding $L_{3}(2)$-complement. Therefore modulo $A$ the group $F$ contains exactly two classes of complements with representatives $T$ and $T^{e}$. Next, for $S$ being one of these representatives and $\beta \in\{x, y, e\}$ the group $A(\beta) S \cong 2^{3}: L_{3}(2)$ contains two classes of complements. Let $S_{\beta}$ be a complement, which is not in the class of $S$. Then

$$
\mathcal{T}=\left\{T, T_{x}, T_{y}, T_{e}, T^{e}, T_{x}^{e}, T_{y}^{e}, T_{e}^{e}\right\}
$$

is a complete set of representatives of the classes of $L_{3}(2)$ complements and the result follows. 
Next we discuss the possibilities for the action of $t \in G\{x, y\} \backslash G(x, y)$ on the classes of $L_{3}(2)$-complements to $P$ in $F$ (sometimes we identify a complement $R \in \mathcal{T}$ with the class it represents). Put $\mathcal{T}_{0}=\left\{T, T_{x}, T_{y}, T_{e}\right\}$ and $\mathcal{T}_{1}=\left\{T^{e}, T_{x}^{e}, T_{y}^{e}, T_{e}^{e}\right\}$.

LEMmA 8.15. Let $i \in\{0,1\}, T_{\alpha}, T_{\beta} \in \mathcal{T}_{i}$ and $\varphi=\varphi(\alpha, \beta)$ be the mapping which sends $k \in T_{\alpha}$ onto $A k \cap T_{\beta}$. Then

(i) $\varphi$ is an isomorphism of $T_{\alpha}$ onto $T_{\beta}$;

(ii) for every $p \in P$ we have $p^{k}=p^{\varphi(k)}$;

(iii) $\varphi$ extends uniquely to an automorphism of $F$ which commutes with $P$.

Proof. (i) is immediate from the proof of Lemma 8.14. Since $A=Z(P)$ by Lemma 8.11 (iii), (ii) follows. Finally (iii) is clear since $F=P T_{\alpha}=P T_{\beta}$.

Let $O^{0}$ be the subgroup in the automorphism group of $F$ which stabilizes the classes of $L_{3}(2)$-complements represented in $\mathcal{T}_{0}$. Then by Lemma $8.15 O^{0}$ permutes transitively the four classes in $\mathcal{T}_{0}$. Furthermore, the results in Lemmas 8.9 to 8.13 are true if instead of $T$ we take any other $L_{3}(2)$-complement, whose class is represented in $\mathcal{T}_{0}$. The next lemma shows that the situation is different for the complements in $\mathcal{T}_{1}$.

Lemma 8.16. $B(x) A / A(x)$ is an indecomposable $T^{e}$-module.

Proof. By the construction $I=B(e)$ maps onto $O_{2}\left(P_{1}\right)$ in $L=$ $G(x) / G_{1}(x)$, while $T$ and $T^{e}$ map onto representatives of two classes of complements to $O_{2}\left(P_{1}\right)$ in $P_{1}$. On the other hand, by Lemmas $8.4,8.7$ and $8.9 B(x) A / A(x)$ is the image in $P$ of $U^{[2]}=V / U$. Now the result is immediate from Lemma 3.16 (i).

LEMMA 8.17. If $t \in G\{x, y\} \backslash G(x, y)$, then the class of $T^{t}$ is contained in $\mathcal{T}_{0}$.

Proof. By Lemma $8.7 t$ permutes $A(x)$ and $A(y)$ as well as $B(x)$ and $B(y)$, normalizing $A(e)$ and $B(e)$. By Lemma 8.12 $B(y) A=J(W) \oplus A(x)$ (as a $T$-module). Therefore

$$
B(x) A=(B(y) A)^{t}=J(W)^{t} \oplus A(y)
$$


as a $T^{t}$-module. If $T^{t} \notin \mathcal{T}_{0}$, then by Lemma 8.15, without loss of generality, we can assume that $T^{t}=T^{e}$. But then the above equality contradicts Lemma 8.16.

Define a mapping $\psi$ of $Y(W)$ onto $J(W)$ which sends $d(s)$ onto $d(s) i(s)$ for every 2-subspace $s$ in $W$. Then clearly $\psi$ is an isomorphism of $T$-modules and it extends uniquely to an automorphism $\psi(T)$ of $F$ which commutes with $T$.

LEMma 8.18. There are four matching (graphs of valency 1) $\Gamma_{S}, S \in$ $\{A(x), A(y), A(e), I\}$ on $\mathcal{T}$. Furthermore,

(i) if $R \in \mathcal{T}_{i}$ and $R^{\prime} \in \mathcal{T}_{j}$ are adjacent in $\Gamma_{S}$, then $i \neq j$ if $S=I$ and $i=j$ otherwise;

(ii) if $a \in O^{0}$, then $\left(\Gamma_{S}\right)^{a}=\Gamma_{S^{a}}$;

(iii) $O^{0} \cong D_{8}$ acts faithfully on $\mathcal{T}_{0}$.

Proof. We assume that the complements in $\mathcal{T}$ share the normalizer $Y$ of a Sylow 7-subgroup isomorphic to $F_{7}^{3}$. Let $X$ be a Sylow 3 -subgroup in this normalizer. Then we define $R, R^{\prime} \in \mathcal{T}$ to be adjacent in $\Gamma_{S}$ if and only if

$$
\left\langle N_{R}(X), N_{R^{\prime}}(X)\right\rangle \cap P \leq S .
$$

Now (i) and (ii) are clear from the proof of Lemma 8.14. Notice that $T$ is adjacent in $\Gamma_{S}$ to $T_{x}, T_{y}, T_{e}$ and $T^{e}$ for $S=A(x), A(y), A(e)$ and $I$, respectively. The automorphism $\psi$ defined before this lemma and the automorphisms $\varphi(\alpha, \beta)$ from Lemma 8.15 generate $D_{8}$ on $\mathcal{T}_{0}$. Since $A(e)=$ $[P, P], \Gamma_{A(e)}$ is stable under $O^{0}$, so (iii) follows.

LEMMA 8.19. The element $t$ induces on $\mathcal{T}$ either the permutation $(T)\left(T_{x}, T_{y}\right)\left(T_{e}\right)\left(T^{e}\right)\left(T_{x}^{e}, T_{y}^{e}\right)\left(T_{e}^{e}\right)$ or the permutation $\left(T_{x}\right)\left(T, T_{e}\right)\left(T_{y}\right)\left(T_{x}^{e}\right)$ $\left(T^{e}, T_{e}^{e}\right)\left(T_{y}^{e}\right)$.

Proof. We know that $t$ permutes $A(x)$ and $A(y)$. Hence it permutes $\Gamma_{A(x)}$ and $\Gamma_{A(y)}$. Now the result is immediate by Lemmas 8.15 (iii) and 8.17.

By Lemma 8.19 the automorphism of $F=G(x, y) / Z(G(x, y))$ induced by $t$ modulo inner automorphisms coincides either with the automorphism $\psi(T)$ defined before Lemma 8.18 or with the automorphism $\psi\left(T_{x}\right)$ defined 
similarly. As in the proof of Lemma 8.18 we assume that the complements in $\mathcal{T}$ share the normalizer $Y$ of a Sylow 7-subgroup (clearly $Y$ is self-normalized in $F$ ) and furthermore that $t$ normalizes $Y$. In this case $t$ permutes the complements in $\mathcal{T}$.

We believe that the full automorphism group of $F$ permutes transitively eight classes of $L_{3}(2)$-complement but this is irrelevant to our main purpose, so in this paper we are not going to investigate this matter further.

\subsection{The amalgam $\mathcal{A}$}

Now it is time to recall that the group $G(x)$ (and hence $G(x, y)$ as well) is specified up to four possibilities. Namely $G(x)$ is a quotient of the covering group $\widehat{H}$ of $G(x) / Z(G(x))$ over a subgroup of order 4 in $Z(\widehat{H})$ which trivially intersects the commutator subgroup of $O_{2}(\widehat{H})$. By the proof of Lemma $8.6 \widehat{H}$ possesses a surjective homomorphism onto the covering group $\widehat{D}$ of $D \cong 2^{6}: L_{4}(2)$. We denote the generators of $Z(\widehat{H})$ by the same letters $c, \pi, z$ as the generators of $Z(\widehat{D})$. Then $c$ generates the commutator subgroup of $\mathrm{O}_{2}(\widehat{H}),\langle c, \pi\rangle=\left[\mathrm{O}_{2}(\widehat{H}), \widehat{H}\right]$ and $z$ generates the centre of the commutator subgroup of the preimage in $\widehat{H}$ of an $L_{4}(2)$-complement in $H$. Notice that the automorphism of $\widehat{D}$ which permutes $\pi$ and $\pi c$ does not extend to an automorphism of $\widehat{H}$ (since the latter group is complete by Lemma 8.15 (iv)). Then the kernel $N$ of the homomorphism of $\widehat{H}$ onto $G(x)$ is one of the following subgroups of $Z(\widehat{H})$ :

$$
\langle z, \pi\rangle,\langle z, \pi c\rangle,\langle z c, \pi\rangle,\langle z c, \pi c\rangle
$$

We emphasize again that the quotients of $\widehat{H}$ over these four subgroups are pairwise non-isomorphic.

Let $\widehat{F}$ be the preimage in $\widehat{H}$ of $G(x, y) / Z_{x}$ and for a subgroup $X \leq F$ let $\widehat{X}$ denote its preimage in $\widehat{F}$. Then $Z(\widehat{F})=\widehat{\langle w\rangle}$ and hence it is of order $2^{4}$. Furthermore, since $L_{4}(2)$ does not preserve non-zero alternating forms on $U$ and $H^{1}\left(L_{4}(2), U^{*}\right)$ is trivial, $\widehat{U} \cong Z(\widehat{H}) \oplus[\widehat{U}, \widehat{H}]$, the latter direct summand being isomorphic to $U$. Thus $Z(\widehat{F})$ is generated by elements $c, \pi, z, \widehat{w}$, where

$$
\langle\widehat{w}\rangle=[\widehat{U}, \widehat{H}] \cap Z(\widehat{F})
$$

We have two possibilities, $\psi(T)$ and $\psi\left(T_{x}\right)$ for the automorphism induced by $t$ on $F$. We are going to employ the condition that this automorphism must extend to an automorphism of $G(x, y)=\widehat{F} / N$ in order to specify $N$ 
and to make the choice between $\psi(T)$ and $\psi\left(T_{x}\right)$. Notice that the extended automorphism must not centralize $\langle c, \pi, z\rangle / N=Z_{x}$.

Let $R \in \mathcal{T}$. Since the Schur multiplier of $R \cong L_{3}(2)$ is of order 2 (cf. Lemma 3.4), $\widetilde{R}:=[\widehat{R}, \widehat{R}]$ is a quotient of $S L_{2}(7)$. Let $k(R)$ denote the generator of $Z(\widehat{R})$ (which is of order 1 or 2 ). First we calculate the elements $k(R)$ for every complement in $\mathcal{T}$.

LEMMA 8.20. In the above terms we have the following: $k(T)=$ $k\left(T_{x}\right)=1, k\left(T_{y}\right)=k\left(T_{e}\right)=\pi, k\left(T^{e}\right)=z, k\left(T_{x}^{e}\right)=z \widehat{w}, k\left(T_{y}^{e}\right)=z c$, $k\left(T_{e}^{e}\right)=z c \widehat{w}$.

Proof. It is convenient to consider $\widehat{H}$ as the subdirect product with respect to $D$ of $H$ and the covering group $\widehat{D}$ of $D \cong 2^{6}: L_{4}(2)$. Then $\widehat{T}$ is the preimage of a Levi complements from a complement to $\mathrm{O}_{2}(D)$ in $D$. Hence arguing as in the proof of Lemma 8.8 we observe that $\widehat{T}$ splits over $Z(\widehat{H})$. Since $U=\langle w\rangle \oplus W$ as a module for $T$, $\widehat{T}$ splits over the whole $Z(\widehat{F})$ and $k(T)=1$. Now as a module for $\widetilde{T} \cong L_{3}(2)$ the preimage $\widehat{A(x)}$ is isomorphic to the direct sum of $Z(\widehat{F})$ and $A(x)$ while $\widehat{A(y)}$ is the direct sum of $\langle c, z, \widehat{w}\rangle$ and an indecomposable extension of $\langle\pi\rangle$ by $A(y)$ (compare the model for $\widehat{D}$ in the proof of Lemma 3.17). By Lemma 3.9 this shows that $k\left(T_{x}\right)$ is trivial while both $k\left(T_{y}\right)$ and $k\left(T_{e}\right)$ are $\pi$ (we can make distinction between $\pi$ and $c \pi$ by declaring that $\langle\pi\rangle=[\widehat{A(y)}, \widetilde{T}])$.

Now let us turn to $T^{e}$. It corresponds to the non-Levi complement to maximal parabolic in an $L_{4}(2)$-complement to $O_{2}(H)$ in $H$. Therefore, firstly $k\left(T_{e}\right)=z$ by Lemma 3.13 , secondly, as $\widetilde{T}_{e}$-modules $\widehat{A(x)}$ is the direct sum of $Z(\widehat{H})$ and an indecomposable extension of $\langle\widehat{w}\rangle$ by $A(x)$ while $\widehat{A(y)}$ is the direct sum of $\langle\pi, c, \widehat{w}\rangle$ and an indecomposable extension of $\langle c\rangle$ by $A(y)$. Now another application of Lemma 3.9 completes the proof.

LEMma 8.21. The element $t$ can not induce the automorphism $\psi(T)$.

Proof. Suppose to the contrary that $t$ induces $\psi(T)$ and try to extend it to $\widehat{F} / N$ for $N$ being one of the above four subgroups. Since $\psi(T)$ maps $T_{x}$ onto $T_{y}$ we immediately conclude that $\pi \in N$. We claim that $z \in N$. Suppose not, then $z c \in N$. But $z w$ could not be in $N$, since $\langle c, w\rangle$ maps bijectively onto the centre of $G(x, y)$. This contradicts Lemma 8.20 since $\psi(T)$ maps $T_{x}^{e}$ onto $T_{y}^{e}$. Thus $N=\langle\pi, z\rangle$. By the proof of Lemma 8.20 in this case in the quotient $\widehat{F} / N$ the preimage of $A$ splits and we can 
factor it out and consider the subgroup $\bar{F}$ which is a central extension of $Y=Z(\widehat{F}) / N$ (of order 4 ) by $F / A$. Since $z \in N$, the preimage of $B(e)$ splits over $Y$ as a module for the image of $T$ while the preimage of $B(x)$ does not split over $\langle c\rangle N / N$ as we can see from the model of $\widehat{D}$. Hence the commutator of $T$ with $B$ intersects $Z(\widehat{F}) / N$ in $\langle c\rangle N / N$ which is impossible, since then $t$ must normalize the latter.

LEMMA 8.22. The following assertions hold.

(i) $t$ induces the automorphism $\psi\left(T_{x}\right)$;

(ii) $N=\langle\pi, z c\rangle$.

Proof. (i) is immediate from Lemmas 8.19 and 8.21. Since $\psi\left(T_{x}\right)$ maps $T$ onto $T_{e}$, we have $\pi \in N$ by Lemma 8.20. Since $\psi\left(T_{x}\right)$ maps $T^{e}$ onto $T_{e}^{e}$, the extension $\psi\left(T_{x}\right)$ must map $\langle z\rangle N$ onto $\langle z c \widehat{w}\rangle N$. Since either $z$ or $z c$ is in $N$, the result follows.

Finally we have arrived to the main result of the section.

Proposition 8.23. In the case (iv) of Proposition 4.1 the amalgam $\mathcal{A}$ is isomorphic to the amalgam $\mathcal{A}_{4}^{(3)} \cong \mathcal{A}\left(\mathrm{Co}_{2}, \Gamma\left(\mathcal{G}\left(\mathrm{Co}_{2}\right)\right)\right.$.

Proof. By Lemma $8.22 N$ is determined which specifies $G(x)$ up to isomorphism and hence $G(x, y)$ as a subgroup of $G(x)$. The action of $t$ on $G(x, y) / Z(G(x, y))$ coincides with $\psi\left(T_{x}\right)$ and the action of $t$ on $Z(G(x, y))$ is clear from the proof of Lemma 8.22. Since $G(x, y)$ does not split over its centre the action of $t$ on the whole $G(x, y)$ is determined. We claim that $t$ can be chosen to be an involution. Indeed by the construction $t^{2}$ induces the trivial automorphism hence either $t^{2}=1$ or $t^{2}$ is the unique element in $Z(G(x, y))$ centralized by $t$. In the latter case instead of $t$ we take at where $a$ is an involution in $Z(G(x, y))$ not centralized by $t$.

\section{§9. $J_{4}$-type amalgams}

In this section we deal with the case (v) in Proposition 4.1. Therefore, we assume that $n=4, G_{1}(x) / G_{2}(x) \cong U^{[2]}, G_{2}(x) / G_{3}(x) \cong U, G_{3}(x) \cong$ $U^{[3]} \cong U^{*}$, where $U$ is the natural module for $L=G(x) / G_{1}(x) \cong L_{4}(2)$.

It was announced in [Tr00] that in the considered situation the structure of the vertex stabilizer is specified up to isomorphism as follows. 
Proposition 9.1. Let $H \cong L_{5}(2), \bar{U}$ be a natural module of $H, V$ be the exterior square of the dual of $\bar{U}$. Let $\bar{W}$ be a hyperplane in $\bar{U}$ and $H(\bar{W}) \cong 2^{4}: L_{4}(2)$ be the (set-wise) stabilizer of $\bar{W}$ in $H$. Then the vertex stabilizer $G(x)$ in a locally projective action corresponding to the case (v) in Proposition 4.1 is isomorphic to the semidirect product of $V$ and $H(\bar{W})$ with respect to the natural action.

It is easy to check (compare Lemmas 3.2 and 3.16) that if we put $R=O_{2}(H(\bar{W}))$, then $C_{V}(R)$ is the only proper $H(\bar{W})$-invariant submodule in $V$ and that $R$ and $C_{V}(R)$ are dual to each other. We have

$$
\begin{gathered}
G_{3}(x)=C_{V}(R) ; \\
G_{2}(x)=C_{V}(R) R ; \\
G_{1}(x)=V R .
\end{gathered}
$$

Therefore $R \cong U$ while $\bar{W} \cong C_{V}(R) \cong U^{*}$, in particular $G(x, y)$ is the centralizer in $G(x)$ of an involution from $R$.

Since we know $G(x)$ up to isomorphism and $G(x, y)$ up to conjugation in $G(x)$, in order to apply Lemmas 4.2 and 4.3 we need to study the automorphism group of $G(x, y)$. We start with the following

Lemma 9.2. The subgroup $V$ is the unique largest normal elementary abelian subgroup in $G(x)$.

Proof. Suppose that $F$ is an elementary abelian normal subgroup in $G(x)$, which is not contained in $V$. Then $V F / V$ is a non-identity normal 2-subgroup in $H(\bar{W}) V / V \cong 2^{4}: L_{4}(2)$, hence it must coincide with $R V / V$. But then $F \cap V$ is contained in $C_{V}(R)$, which is only 4-dimensional. Hence $|F| \leq 2^{8}$ (in fact $G_{2}(x)=R C_{V}(R)$ attains this bound) and the result follows.

In view of Lemma 4.3 the following result is useful.

Lemma 9.3. The group $G(x)$ is complete.

Proof. Let $M \cong L_{4}(2)$ be a complement to $R$ in $H(\bar{W})$. Then by Lemma $9.1 M$ is a complement to $G_{1}(x)=O_{2}(G(x))$ in $G(x)$. By Lemma 9.2 $V$ is characteristic in $G(x)$. Suppose first that $\alpha$ preserves the class of $M$. Then we can assume that $\alpha$ normalizes $M$. Since $V$ involves $U^{*}$ but not $U$, $\alpha$ induces an inner automorphism of $M$ and hence we can assume that $\alpha$ 
commutes with $M$. Since $V$ is a direct sum of a non-isomorphic $M$-modules (isomorphic to $U^{*}$ and $\left.U^{[2]}\right), \alpha$ must centralize $V$. Also $G_{3}(x) R$ is abelian, normalized by $M$. Since $O_{2}(G(x)) / G_{3}(x)$ is a direct sum of non-isomorphic $M$-modules, we see that $G_{3}(x) R$ is normalized by $\alpha$. But $G_{3}(x) R$ is also a direct sum of non-isomorphic $M$-modules, hence it is centralized by $\alpha$ and the latter is the identity automorphism. Now suppose that the class of $M$ is not preserved by $\alpha$. By Lemma $3.2 G(x)$ contains at most two classes of complements to $\mathrm{O}_{2}(G(x))$. In fact a complement not in the class of $M$ can be obtained as the image of $M$ under an outer automorphism $\sigma$ of $D:=2^{6}: L_{4}(2)$ which permutes two classes of complements to $O_{2}(D)$ in $D$. We claim that $\sigma$ can not be extended to an automorphism of the whole $G(x)$. Indeed, consider the action of $D$ on $G_{3}(x) R$. Under the action of $\mathrm{O}_{2}(D)$ there are 64 images of $R$ and $M^{\sigma}$ acting on these images has two orbits with lengths 8 and 56 (compare Lemma 3.14). Therefore $G_{3}(x) R$ is indecomposable as $M^{\sigma}$-module and the claim follows.

Let us turn to the subgroup $G(x, y)$. It is immediate form Lemma 9.1 that $G(x, y)$ splits over $\mathrm{O}_{2}(G(x, y))$, but we prefer to deal with a specific complement $T$ (which we called a Levi complement):

$$
T=H(\bar{w}) \cap H(\bar{W}) \cap H\left(\bar{W}_{1}\right) \cap H\left(\bar{w}_{1}\right),
$$

where $\bar{w}$ is a vector from $\bar{W}, \bar{w}_{1}$ is a vector from $\bar{U} \backslash \bar{W}$ and $\bar{W}_{1}$ is a hyperplane in $\bar{U}$ containing $\bar{w}_{1}$, but not $\bar{w}$. Notice that $\bar{W}$ and $\bar{W}_{1}$ are considered as 1 -subspaces in $\bar{U}^{*}$ while $\bar{w}$ and $\bar{w}_{1}$ are hyperplanes in $\bar{U}^{*}$. In what follows the 3 -dimensional subspace $\bar{w} \cap \bar{w}_{1}$ of $\bar{U}^{*}$ will be called the natural module of $T$.

Lemma 9.4. As a T-module, $V=V_{0} \oplus V_{1} \oplus V_{2} \oplus V_{3}$, where $V_{0}$ and $V_{1}$ are the natural modules, $V_{2}$ is the dual natural module and $V_{3}$ is 1-dimensional trivial module.

Proof. Since $V=\left(\bar{U}^{*}\right)^{[2]}$ we can use notation similar to those in Lemma 3.1. In these terms

$$
\begin{array}{ll}
V_{0}=V(\bar{w}) \cap V(\bar{W}), & V_{1}=V\left(\bar{w}_{1}\right) \cap V\left(\bar{W}_{1}\right), \\
V_{2}=V(\bar{w}) \cap V\left(\bar{w}_{1}\right), & V_{3}=V(\bar{W}) \cap V\left(\bar{W}_{1}\right) .
\end{array}
$$


As we have already mentioned, $G(x, y)$ is the centralizer in $G(x)$ of an element $r \in R^{\#}$ and we can chose $r$ acts on $\bar{U}$ as the transvection whose axis is $\bar{W}$ and whose centre is $\bar{w}$. Notice that $H(\bar{w}) \cong 2^{4}: L_{4}(2)$ and we put $S=O_{2}(H(\bar{w}))$. Then the above introduced subgroup $T \cong L_{3}(2)$ is a Levi complement to $R$ in $M \cong L_{4}(2)$ as in the proof of Lemma 9.3 and also a Levi complement in the $L_{4}(2)$-complement to $S$ in $H(\bar{w})$.

The following result is immediate from the definition.

LEMMA 9.5. The following assertions hold:

(i) the subgroup $G(x, y)$ is the semidirect product with respect to the natural action of $V \cong 2^{10}$ and $P:=C_{H}(r) \cong 2_{+}^{1+6}: L_{3}(2)$;

(ii) $\mathrm{O}_{2}(P)=R S, T$ is a complement to $\mathrm{O}_{2}(P)$ in $P$;

(iii) $Z\left(O_{2}(G(x, y))\right)=C_{V}\left(O_{2}(P)\right)$ and it is the dual natural T-module;

(iv) considering as T-modules, $R$ is the direct sum of 1-dimensional trivial and the natural module, while $S$ is direct sum of 1-dimensional and the dual natural module.

We will also adopt a different point of view on $G(x, y)$. This is based on the observations that the semidirect product of $H \cong L_{5}(2)$ and $V$ is the vertex stabilizer in a locally projective action of type $(5,2)$, corresponding to the case (ii) in Proposition 4.1. So there is a group $\bar{G}$ acting locally projectively on the tree $\bar{\Gamma}$ of valency 31 such that $\bar{G}(\bar{x}) \cong V: H$ for a vertex $\bar{x} \in \bar{\Gamma}$. It worth mentioning that by Lemma 6.6 the amalgam $\mathcal{A}=\{\bar{G}(\bar{x}), \bar{G}\{\bar{x}, \bar{y}\}\}$ associated with this action is isomorphic to either $\mathcal{A}\left(O_{10}^{+}(2), D^{+}(10,2)\right)$ or $\mathcal{A}_{5}^{(1)}$ (the latter amalgam possesses the fourth Janko group $J_{4}$ as a constrained completion). In these terms $G(x)$ is a subgroup of index 31 in $\bar{G}(\bar{x})$ and since $\bar{U}$ is the natural module of $\bar{G}(\bar{x}) / V$ associated with the action of $\bar{G}$ on $\bar{\Gamma}, G(x)$ is the stabilizer in $\bar{G}(\bar{x})$ of the geometric subgraph $\bar{\Sigma}^{[4]}$ of valency 15 in $\bar{\Gamma}$, which corresponds to the hyperplane $\bar{W}$ of $\bar{U}$. Thus

$$
G(x)=\bar{G}^{[4]}(\bar{x}) \text { and } G(x, y)=\bar{G}^{[4]}(\bar{x}, \bar{y}),
$$

where $\bar{y}$ is a suitable vertex in $\bar{\Sigma}^{[4]}$ adjacent to $\bar{x}$ (in particular $G(x, y)$ is a subgroup of index 15 in $\bar{G}(\bar{x}, \bar{y}))$. According to Proposition 6.1

$$
\bar{G}(\bar{x}, \bar{y})=Z A B K
$$

where $V=B Z, A=S=O_{2}(H(\bar{w})), K \cong L_{4}(2)$ is a complement to $\bar{A}$ in $H(\bar{w})$. Furthermore $\{A Z, B Z, C Z=C\}$ is the complete set of normal 
elementary abelian subgroups of order $2^{10}$ in $\bar{G}(\bar{x}, \bar{y})$ where (as $K$-modules) $A Z=A \oplus Z$ and $B Z=B \oplus Z$, while $C$ is the only indecomposable extension of the exterior square of the natural module by the natural module (compare Lemma 8.1). We also have the following

LEMMA 9.6. In the above terms $G(x, y)$ is the centralizer in $\bar{G}(\bar{x}, \bar{y})$ of a non-identity element $a \in A$.

We proceed to calculating the automorphisms of $G(x, y)$. First we decide what can be the image of $V$ under such an automorphism.

LEMMA 9.7. Let $F$ be a normal elementary abelian subgroup of order $2^{10}$ in $G(x, y)$. Then $F$ is either $A Z$, or $B Z$, or $C Z$.

Proof. Let $\chi$ be the natural homomorphism of $G(x, y)$ onto $G(x, y) / V$. Suppose that $F$ is as in the hypothesis and $F \neq V$. Then $\chi(F)$ is a nontrivial elementary abelian normal subgroup in

$$
\chi(G(x, y))=\chi(P) \cong P \cong 2_{+}^{1+6}: L_{3}(2) .
$$

By Lemma 9.5 one of the following holds: (a) $X=\chi(R \cap S)$ (of order 2); (b) $X=\chi(R)$; (c) $X=\chi(S)$. Now (a) is impossible since $H$ is not generated by transvections on $V$; (b) is impossible since $C_{V}(R)$ is 4-dimensional, so (c) holds. Since $C_{V}(S)$ is 6-dimensional, $F \cap V=C_{V}(S)=Z$. Since $G(x, y)$ induces on $A$ and $B$ indecomposable action isomorphic to $2^{3}: L_{3}(2)$, there is still only one $G(x, y)$-invariant isomorphism of $A$ onto $B$ and the result follows.

Put $E:=G(x, y) / Z$, so that $E \cong(Z A B / Z) J$, where $J=(K \cap$ $G(x, y)) Z / Z \cong 2^{3}: L_{3}(2)$ and $T Z / Z$ is a complement to $O_{2}(J)$ in $J$. Then (as $T$-modules) we have

$$
A Z / Z=A^{(1)} \oplus A^{(3)}, \quad B Z / Z=B^{(1)} \oplus B^{(3)}, \quad C Z / Z=C^{(1)} \oplus C^{(3)},
$$

where $X^{(i)}$ is $i$-dimensional for $X=A, B$ or $C$ and $i=1$ and 3 . Put $D^{(3)}=O_{2}(J)$. Since $\bar{G}(\bar{x}, \bar{y}) / Z \cong\left(2^{4} \oplus 2^{4}\right): L_{4}(2)$, it is easy to see the following

Lemma 9.8. Let $\mathrm{N}$ be the commutator subgroup of $\mathrm{O}_{2}(E)$. Then

(i) $N$ is elementary abelian of order $2^{2}$ generated by $A^{(1)}$ and $B^{(1)}$; 
(ii) $E / N$ is elementary abelian, isomorphic (as a $T$-module) to the direct sum $A^{(3)} \oplus B^{(3)} \oplus D^{(3)}$, where $A^{(3)}$ and $B^{(3)}$ are the natural modules and $D^{(3)}$ is the dual natural module;

(iii) $E$ contains three normal extraspecial subgroups of type $2_{+}^{1+6}$; they are $\left\langle Y^{(3)}, D^{(3)}\right\rangle$ for $Y=A, B$ and $C$ and their centres are $A^{(1)}, B^{(1)}$ and $C^{(1)}$, respectively.

Let us study the classes of $L_{3}(2)$-complements to $O_{2}(E)$ in $E$. Abusing the notation we identify $T$ with its image in $E$, so $T$ is one of the complements we are after.

LEMмA 9.9. The following assertions hold:

(i) E contains exactly five classes of $L_{3}(2)$-complements;

(ii) suppose that $T^{\prime}$ is a complement such that for $X \in\{A, B, C, D\}$ the module $\left\langle A^{(1)}, B^{(1)}, X^{(3)}\right\rangle$ is completely reducible as $T^{\prime}$-modules, then $T^{\prime}$ is a conjugate of $T$;

(iii) if $\sigma$ is an automorphism of $G(x, y)$, then for an inner automorphism $\alpha$ we have $T^{\sigma} Z=T^{\alpha} Z$.

Proof. Since $\mathrm{O}_{2}(E)$ contains three 3-dimensional chief factors and two 1-dimensional ones, $E$ contains at most eight classes of complements by Lemma 3.2. Furthermore, since the 1-dimensional factors are in $N$ and $\mathrm{O}_{2}(E) / N$ is abelian, $E / N$ contains exactly eight classes of complements. The preimage in $E$ of an $L_{3}(2)$-subgroup from $E / N$ might or might not split over $N$. Since $T$ normalizes $X^{(3)}$ for $X=A, B, C$ or $D$, the image of $T$ under an outer automorphism of $X^{(3)} T \cong 2^{3}: L_{3}(2)$ is a complement to $\mathrm{O}_{2}(E)$, so $E$ contains at least five classes of complements. On the other hand it is easy to see using Lemma 3.9 that $\left\langle Y^{(3)}, D^{(3)}\right\rangle \cong 2_{+}^{1+6}: L_{3}(2)$ contains only three classes of $L_{3}(2)$-complements and one class of $S L_{2}(7)$ subgroups (here $Y=A, B$ or $C$ ), hence (i) follows. By the above argument the representatives of the classes of complements are $T$ and its images $T^{\xi}$ under outer automorphisms $\xi$ of $X^{(3)} T \cong 2^{3}: L_{3}(2)$, where $\xi$ will be denoted by $\alpha, \beta, \gamma$ and $\delta$ for $X=A, B, C$ and $D$, respectively. Now it is easy to deduce from Lemma 3.12 that $T^{\xi}$ acts indecomposably on $\left\langle D^{(3)}, A^{(1)}\right\rangle$, $\left\langle D^{(3)}, B^{(1)}\right\rangle,\left\langle D^{(3)}, C^{(1)}\right\rangle$ and $\left\langle A^{(3)}, A^{(1)}\right\rangle$ for $\xi=\alpha, \beta, \gamma$ and $\delta$, respectively. Hence (ii) follows. By Lemma $9.7 \sigma$ normalizes $Z=A Z \cap B Z \cap C Z$ and so it acts on $E$, now by (ii) $\sigma$ preserves the class of $T$ in $E$, so (iii) follows.

LEMMA 9.10. The following assertions hold: 
(i) as a T-module $C Z$ involves the indecomposable extension of the dual natural module by the natural module;

(ii) if $\sigma$ is an automorphism of $G(x, y)$, then $V^{\sigma} \in\{V=B Z, A Z\}$.

Proof. By Lemma 6.1 (e) $C=C Z$ as a module for the complement $K \cong L_{4}(2)$ to $A=O_{2}(H(\bar{w}))$ in $H(\bar{w})$ is an indecomposable extension of the exterior square of the natural module by the natural module. This extension is unique by Lemma 8.1 and it is a quotient of the $G F(2)$-permutation module of $K$ on the set of non-zero vectors in the natural module. Since $T$ is a Levi complement in $K$ (i) can be checked by direct calculation (dualize Lemma 8.4 (iv)). By Lemma 9.7 $V^{\sigma} \in\{A Z, B Z, C Z\}$. Clearly the action of $T$ on $V$ is isomorphic to the action of $T^{\sigma}$ on $V^{\sigma}$. Since $Z=A Z \cap B Z \cap C Z$, by Lemma 9.9 (iii) the actions of $T$ and $T^{\sigma}$ in $V^{\sigma}$ are isomorphic. Finally, the action of $T$ on $V$ is completely reducible by Lemma 9.4 while by (i) on $C Z$ it is not. Hence (ii) follows.

By Lemma 6.4 Out $\bar{G}(\bar{x}, \bar{y})$ is elementary abelian of order $2^{2}$ generated by the automorphism $\tau_{0}$ and $\tau_{1}$ defined in the paragraph after the proof of Lemma 6.4. Each of these two automorphisms normalizes $G(x, y)$ and we denote by $\sigma_{0}$ and $\sigma_{1}$ the automorphisms of $G(x, y)$ induced by $\tau_{0}$ and $\tau_{1}$, respectively. A further automorphism of $G(x, y)$ can be constructed as follows. By Lemma 9.5 (iii) $V_{z}:=Z\left(O_{2}(G(x, y))\right.$ is a natural module for $T$. Let $\sigma_{2}$ be an automorphism of $G(x, y)$ which centralizes $O_{2}(G(x, y))$ and induces an outer automorphism of $V_{z} T \cong 2^{3}: L_{3}(2)$ (it must be clear that $\sigma_{2}$ is indeed an automorphism).

LEMMA 9.11. Out $G(x, y)$ is elementary abelian of order $2^{3}$ generated by the images of $\sigma_{0}, \sigma_{1}$ and $\sigma_{2}$.

Proof. We have $G(x, y)=Z A B D T$, where $T$ is the Levi complements, $D=O_{2}(K)$ the dual natural module for $T, V=B Z$ and $Z$ is the direct sum of the natural and the dual natural $T$-modules. Let $\sigma$ be an automorphism of $G(x, y)$. By Lemma 9.10 (ii) and since $\tau_{0}$ maps $B Z$ onto $A Z$, by multiplying $\sigma$ by $\sigma_{0}$ we can assume that $\sigma$ normalizes each of $B Z, A Z$ and $Z$. By Lemma 9.9 (iii) we can adjust $\sigma$ by inner automorphisms so that it commutes with the actions of $T$ on $V$ and on $A Z$. By Lemma 9.4 as a $T$-module $V$ is a direct sum of irreducible submodules: $V_{i}, i=0,1,2,3$. Furthermore among these modules there is only one pair $\left(V_{0}\right.$ and $\left.V_{1}\right)$ of isomorphic ones and $V_{0}$ is contained in $Z$. Let $V_{1 / 2}$ be the third $T$-submodule 
in $V$ isomorphic to the natural module (the diagonal of $V_{0}$ and $V_{0}$ ). Then there is a unique linear transformation $\lambda$ which commutes with the action of $T$ and stabilizes $Z$, in particular $\lambda$ maps $V_{1}$ onto $V_{1 / 2}$. But in fact $C_{H}(T)$ is of order (at least) 2 since it contains the element $r$ as in Lemma 9.5 and hence $r$ induces the above transformation $\lambda$. So, by multiplying (if necessary) $\sigma$ by $\lambda$ (which is inner), we achieve the trivial action of $\sigma$ on both $V=B Z$ and $A Z$.

Now let us consider the subgroup $I=Z D T \cong 2^{6}: 2^{3}: L_{3}(2)$. By the construction this is an index 15 subgroup of the semidirect product of $K \cong L_{4}(2)$ and $Z$ which the the exterior square of the natural module for $K$. So it is easy to observe that $I$ is exactly the group from Lemma 6.3. By that lemma the stabilizer of $Z$ in the outer automorphism group of $I$ is of order 4 . On the other hand it is easy to see that $\sigma_{0}$ centralizes $I$ while $\sigma_{1}$ and $\sigma_{2}$ induce different outer automorphism of $I$. Thus in order to complete the proof it is sufficient to show that with $\sigma$ adjusted as above $I^{\sigma}=I$. This is true, since $Z^{\sigma}=Z, T^{\sigma} Z=T Z$ and, finally, $D$ is the dual natural module of $T$ and every chief such factor of $G(x, y)$ from $Z A B$ is contained in $Z$. So the proof is complete in view of an easy observation that the images of $\sigma_{0}, \sigma_{1}$ and $\sigma_{2}$ indeed generate an elementary abelian subgroup of order 8 in Out $G(x, y)$.

Now we are well prepared to prove the main result of the section.

Proposition 9.12. Let $\mathcal{A}=\{G(x), G\{x, y\}\}$ be the amalgam associated with a locally projective action which corresponds to case (v) in Proposition 4.1. Let $I=\operatorname{Inn} G(x, y)$ and $\sigma$ be the automorphism of $G(x, y)$ induced by $t \in G\{x, y\} \backslash G(x, y)$. Then one of the following holds:

(i) $\sigma \in I \sigma_{1}$ and $\mathcal{A} \cong \mathcal{A}_{4}^{(4)} \cong \mathcal{A}\left(J_{4}, \Gamma\left(\mathcal{G}\left(J_{4}\right)\right)\right.$;

(ii) $\sigma \in I \sigma_{1} \sigma_{2}$ and $\mathcal{A} \cong \mathcal{A}_{4}^{(5)}$.

Proof. By Lemma 9.11 there are eight possibilities for the image of $\sigma$ in Out $G(x, y)$. Thus we may (and will) assume that $\sigma=\sigma_{0}^{\alpha} \sigma_{1}^{\beta} \sigma_{2}^{\gamma}$, where $\alpha, \beta, \gamma \in\{0,1\}$. Since $\mathcal{A}$ must be simple, $\sigma$ should not normalize subgroups in $G(x, y)$ normal in $G(x)$.

Remark. Since $\sigma_{2}$ acts trivially on $O_{2}(G(x, y))$, a subgroup in $O_{2}(G(x$, $y))$ is normalized by an automorphism $\delta$ if and only if it is normalized by $\delta \sigma_{2}$. 
Clearly $\sigma_{2}$ and the identity automorphism must excluded. By the construction $\sigma_{0} \sigma_{1}$ normalizes $V$, hence it also must be excluded together with $\sigma_{0} \sigma_{1} \sigma_{2}$.

We claim that $\sigma_{0}$ normalizes $C_{V}(R) R=G_{2}(x)$. This can be checked directly but also follows from the following argument. Let $\bar{G} \cong O_{10}^{+}(2)$ acts on the corresponding dual polar graph $\bar{\Gamma}=D^{+}(10,2)$. Then an element $\bar{t} \in \bar{G}\{\bar{x}, \bar{y}\} \backslash \bar{G}(\bar{x}, \bar{y})$ induces on $\bar{G}(\bar{x}, \bar{y})$ the automorphism $\tau_{0}$ and hence on $G(x, y)$ it induces $\sigma_{0}$. On the other hand, $G(x)$ is the stabilizer of a geometric subgraph $\bar{\Sigma}^{[4]}$ and the latter subgraph is also stabilized by $\bar{t}$. Hence $\bar{t}$ normalizes the vertex-wise stabilizer $\bar{G}_{-}^{[4]}$ of $\bar{\Sigma}^{[4]}$ in $\bar{G}$ which is an elementary abelian subgroup of order $2^{8}$, normal in $G(x)$. By the order consideration, or otherwise it is clear that $\bar{G}_{-}^{[4]}=C_{V}(R) R$. By the above remark the latter subgroup is also normalized by $\sigma_{0} \sigma_{2}$.

Since both $\mathcal{A}_{5}^{(1)}$ and $\mathcal{A}_{4}^{(4)}$ have $J_{4}$ as their faithful completions, it is by the construction that the automorphism $\sigma_{1}$ is realized in the latter amalgam. By the above remark $\sigma_{1} \sigma_{2}$ does not normalize subgroups in $G(x, y)$ normal in $G(x)$ and by Lemma $4.3 \sigma_{1}$ and $\sigma_{1} \sigma_{2}$ lead to non-isomorphic amalgam. Finally it is clear that the squares of $\sigma_{1}$ and $\sigma_{1} \sigma_{2}$ induce the trivial automorphism. Since the centre of $G(x, y)$ is trivial, $t^{2}$ must be the identity, which completes the proof.

A completion of $\mathcal{A}_{4}^{(5)}$ in $A_{256}$ which is constrained at level 2 was constructed in [IP04].

\section{§10. $B M$-type amalgam}

Here we study the last remaining case (vi) in Proposition 4.1: $n=5$, $G_{1}(x) / G_{2}(x) \cong U^{[3]} ; G_{2}(x) / G_{3}(x) \cong U^{[2]}, G_{3}(x) / G_{4}(x) \cong U, G_{4}(x) \cong U^{[4]}$. We show that in this case the amalgam $\mathcal{A}$ is isomorphic to the one associated with the action of the Baby Monster sporadic simple group $B M$ on the derived graph of its Petersen type geometry of rank 5.

The flavour of our treatment of this case differ from that of the other cases. One of the reasons for this is the complexity of the structure of the vertex stabilizer. An information on certain 'sections' of $G(x)$ was recorded in [Tr03a] but it is still not clear how many isomorphism types fit into the picture.

Instead of identifying the vertex stabilizer, we use ideas from the characterization of the amalgams determined by the locally projective actions of type $(2, q)$ which are $(s+1)$-transitive for $s \geq 3$ (cf. [W79] and [DS85]). 
On the final step of that characterization it was shown that the underlying tree possesses a folding onto a generalized $s$-gon and the folding commutes with the action of the group (the $(s+1)$-arc-transitive generalized $s$-gons were classified long before that).

To implement this strategy in full, one would show that in the considered situation the tree possesses a folding onto the derived graph of a $P$-geometry, commuting with the action of the group and then apply the classification of the flag-transitive $P$-geometries achieved in [Iv99] and [IS02]. Right now we don't know how to accomplish this. Instead, using induction we show that every proper geometric subgraph in the tree possesses such a folding which commutes with the action of its stabilizer in $G$. This supplies us with an amalgam which 'looks like' the amalgam of maximal parabolics associated with a flag-transitive action on a $P$-geometry. Finally, extending the methods developed in [IS02] we show that the amalgam is unique up to isomorphism and therefore indeed comes from a $P$-geometry of rank 5 .

We should emphasize that in this case an independent identification of the structure of the vertex stabilizer (which is a very interesting problem) is still missing. In this respect our approach is rather conceptual (according to [B84] 'one proof is said to be more conceptual than another if it gives less information about the objects being studied').

\subsection{Geometric subgraphs}

Let $\Gamma$ be a graph and $G$ be a group acting on $\Gamma$ locally projectively and corresponding to case (vi) in Proposition 4.1 (in particular $\Gamma$ is of valency 31). We adopt notation from Section 2. We assume that $\Gamma$ contains a complete family of geometric subgraphs (which is always true when $\Gamma$ is a tree). Let

$$
\{x\} \subset\{x, y\} \subset \Sigma^{[2]} \subset \Sigma^{[3]} \subset \Sigma^{[4]}
$$

be a maximal flag in the geometry $\mathcal{G}$ associated with the action of $G$ on $\Gamma$, where $\Sigma^{[i]}$ is a geometric subgraph of valency $2^{i}-1$ for $2 \leq i \leq 4$. If $\Delta^{[i]}=\Sigma^{[i]}(x)$, then

$$
\{y\} \subset \Delta^{[2]} \subset \Delta^{[3]} \subset \Delta^{[4]}
$$

is a maximal flag in the projective space structure $\Pi_{x}$ on $\Gamma(x)$, preserved by $G(x)$. Let

$$
\left\{G^{[0]}=G(x), G^{[1]}=G\{x, y\}, G^{[2]}, G^{[3]}, G^{[4]}\right\}
$$


be the amalgam of maximal parabolics associated with the action of $G$ on $\mathcal{G}$, where $G^{[i]}$ is the (global) stabilizer of $\Sigma^{[i]}$ in $G$ (recall that the vertex-wise stabilizer of $\Sigma^{[i]}$ is denoted by $\left.G_{-}^{[i]}\right)$. Let

$$
\mathcal{A}^{[i]}=\left\{G^{[i]}(x) / G_{-}^{[i]}, G^{[i]}\{x, y\} / G_{-}^{[i]}\right\}
$$

be the amalgam associated with the action of $G^{[i]}$ on $\Sigma^{[i]}$.

LEMMA 10.1. The following assertions hold:

(i) $\mathcal{A}^{[4]} \cong \mathcal{A}_{4}^{(3)} \cong \mathcal{A}\left(\mathrm{Co}_{2}, \Gamma\left(\mathcal{G}\left(\mathrm{Co}_{2}\right)\right)\right.$;

(ii) $\mathcal{A}^{[3]} \cong \mathcal{A}_{3}^{(5)} \cong \mathcal{A}\left(\right.$ Aut $\left.M_{22}, \Gamma\left(\mathcal{G}\left(M_{22}\right)\right)\right)$.

(iii) $\mathcal{A}^{[2]} \cong\left\{S_{3} \times 2, D_{8}\right\}$.

Proof. By Lemma 4.2 in [Tr01] $\mathcal{A}^{[4]}$ corresponds to case (iv) in Proposition 4.1 and hence (i) follows from Proposition 8.23. Since $\mathcal{G}\left(M_{22}\right)$ is the point residue in $\mathcal{G}\left(\mathrm{Co}_{2}\right)$, (i) implies (ii). Now (iii) is quite clear.

LEMMA 10.2. The following assertions hold:

(i) $G_{-}^{[4]}=G_{0}^{[4]}$ is of order $2^{23}$;

(ii) $G_{-}^{[4]} G_{1}(x) / G_{1}(x) \cong 2^{4}$;

(iii) $\left(G_{-}^{[4]} \cap G_{i}(x)\right) G_{i+1}(x) / G_{i+1}(x) \cong 2^{n_{i}}$ where $n_{i}=4,6,4$ and 5 for $i=1,2,3$ and 4 , respectively;

(iv) $G_{-}^{[4]}$ is non-abelian.

Proof. The group $G^{[4]}(x)$ contains $G_{1}(x)$ with $G^{[4]}(x) / G_{1}(x) \cong 2^{4}$ : $L_{4}(2)$, and by Lemma 10.1 (i) $G^{[4]}(x)$ induces on $\Sigma^{[4]}$ the group $2^{1+4+6}$ : $L_{4}(2)$ (compare Table 1), thus we obtain (i) from our main assumption and the order consideration. Since $G^{[4]}(x) / G_{1}(x)$ is the stabilizer of $\Delta^{[4]}$ in $L \cong L_{5}(2)$ and $2^{4}: L_{4}(2)$ is not a homomorphic image of $G^{[4]}(x) / G_{-}^{[4]} \cong$ $2^{1+4+6}: L_{4}(2)$ (compare Lemma 8.5 (iii)), (ii) follows. Since $U^{[i]}\left(\Delta^{[4]}\right)$ is the only submodule in $U^{[i]}$ stable under $G^{[4]}(x)$, it is easy to deduce (iii) from Lemma 10.1 (i). Notice that in particular we have $G_{4}(x) \leq G^{[4]}(x)$. Since $C_{G(x)}\left(G_{4}(x)\right)=G_{1}(x)$, in view of (ii), this immediately implies (iv).

Since $G_{-}^{[i]} / G_{0}^{[i]} \cong L_{n-i}(2)$, Lemmas 10.1 (ii), (iii) and 10.2 (i) imply the following 
LEMMA 10.3. The following assertions hold:

(i) $G_{-}^{[3]} / G_{0}^{[3]} \cong S_{3} \cong L_{2}(2)$ and $\left|G_{0}^{[3]}\right|=2^{32}$;

(ii) $G_{-}^{[2]} / G_{0}^{[2]} \cong L_{3}(2)$ and $\left|G_{0}^{[2]}\right|=2^{35}$;

(iii) $G_{-}^{[4]} \leq G_{-}^{[3]}$ and $\left[G_{-}^{[4]}: G_{-}^{[4]} \cap G_{0}^{[3]}\right]=2$.

The pair $(\widetilde{G}, \widetilde{\Gamma})$ where $\widetilde{G} \cong B M$ and $\widetilde{\Gamma}$ is the derived graph of the $P$ geometry of $B M$ satisfies the assumption of this section. The information on the structure of the parabolics in $B M$ is summarized in the following lemma (cf. [IS02]).

LEMMA 10.4. If $\widetilde{G}=B M$, then

(i) $\widetilde{G}_{-}^{[4]}=\widetilde{G}_{0}^{[4]} \cong 2_{+}^{1+22}, \widetilde{G}^{[4]} / \widetilde{G}_{-}^{[4]} \cong C o_{2}$ and $C_{\widetilde{G}^{[4]}}\left(\widetilde{G}_{-}^{[4]}\right)=Z\left(\widetilde{G}_{-}^{[4]}\right) \cong 2$;

(ii) $\widetilde{G}^{[3]} / \widetilde{G}_{-}^{[3]} \cong$ Aut $M_{22}$ and $C_{\widetilde{G}^{[3]}}\left(\widetilde{G}_{-}^{[3]}\right)=Z\left(\widetilde{G}_{-}^{[3]}\right)=1$;

(iii) $\widetilde{G}^{[2]} / \widetilde{G}_{-}^{[2]} \cong S_{5}$ and $C_{\widetilde{G}^{[2]}}\left(\widetilde{G}_{-}^{[2]}\right)=Z\left(\widetilde{G}_{-}^{[2]}\right)=1$.

Our nearest goal is to bring the generic example $G$ closer to the model $\widetilde{G} \cong B M$ by proving the following

Proposition 10.5. The centre of $G_{-}^{[i]}$ is trivial for $i=2,3$ and it is of order 2 for $i=4$. The image of $G^{[i]}$ in Out $G_{-}^{[i]}$ is isomorphic to

$$
O_{4}^{-}(2) \cong S_{5}, \text { Aut } M_{22} \text { and } \mathrm{Co}_{2}
$$

for $i=2,3$ and 4 , respectively.

Keeping in mind that $G$ can well be the universal completion of the amalgam formed by the vertex and edge stabilizers in $\widetilde{G}$, one can say that Proposition 10.5 is the best possible generalization of Lemma 10.4. The proof of Proposition 10.5 (to be completed in Subsection 10.4) will be achieved via identification of the images $I^{[i]}$ of the groups $G^{[i]}$ under their natural homomorphisms $\varphi^{[i]}$ into Out $G_{-}^{[i]}$ for $i=2,3$ and 4 .

\subsection{The action of $G^{[3]}$ on $G_{-}^{[3]}$}

Our crucial tool is the knowledge of the amalgam associated with the action of $G^{[4]}$ on $\Sigma^{[4]}$ specified by Lemma 10.1 (i). An important property of this action is the following (compare Table 1). 
LEMma 10.6. Suppose that $F$ acts on $\Lambda$ locally projectively of type $(4,2)$ and the amalgam formed by the vertex and edge stabilizers is isomorphic to $\mathcal{A}_{4}^{(3)}=\mathcal{A}\left(\mathrm{Co}_{2}, \Gamma\left(\mathcal{G}\left(\mathrm{Co}_{2}\right)\right)\right.$. Let $\Theta$ be a geometric subgraph of valency 7 in $\Lambda$. Then the vertex-wise stabilizer $F(\Theta)$ of $\Theta$ is elementary abelian of order $2^{10}$ and the global stabilizer $F[\Theta]$ of $\Theta$ induces on $F(\Theta)$ an irreducible action of Aut $M_{22}$ (more specifically $F(\Theta)$ is the irreducible Golay code module for this action). Furthermore, $F(\Theta)$ acts faithfully on the set of vertices adjacent to $\Theta$.

By the definition $G_{+}^{[3]}$ is the element-wise stabilizer in $G^{[3]}$ of the three geometric subgraphs of valency 15 containing $\Sigma^{[3]}$. Since $\Sigma^{[4]}$ is one of these three subgraphs $G_{+}^{[3]} \leq G^{[4]}$. Furthermore, because of the fact that $G_{-}^{[4]}$ and $G_{+}^{[4]}$ factorize $G^{[4]}$, the action of $G_{+}^{[3]}$ on $\Sigma^{[4]}$ coincides with that of the stabilizer of $\Sigma^{[3]}$ in $G^{[4]}$. Hence Lemma 10.6 implies the following

Lemma 10.7. Let $K=G_{0}^{[3]} \cap G_{-}^{[4]}$ be the kernel of the action of $G_{0}^{[3]}$ on the vertex-set of $\Sigma^{[4]}$. Then the quotient $G_{0}^{[3]} / K$ is elementary abelian of order $2^{10}$ and $G_{+}^{[3]}$ induces on this quotient the irreducible action of Aut $M_{22}$ as on its Golay code module.

There are three geometric subgraphs of valency 15 containing $\Sigma^{[3]}$ and by Lemma 10.7 on each $G_{0}^{[3]}$ induces an elementary abelian group of order $2^{10}$. Let us determine the combined action.

LEMMA 10.8. Let $N$ be the kernel of the action of $G_{0}^{[3]}$ on the union of the geometric subgraphs containing $\Sigma^{[3]}$ and $Q=G_{0}^{[3]} / N$. Then $Q$ is elementary abelian of order $2^{20} ; G^{[3]}$ induces on $Q$ the group Aut $M_{22} \times S_{3}$ and with respect to this action $Q$ is the tensor product of the 10-dimensional Golay code module and the natural module of $S_{3} \cong L_{2}(2)$.

Proof. By Lemmas 10.6 and $10.7 Q$ is elementary abelian and it acts faithfully on the set of vertices adjacent to $\Sigma^{[3]}$. Consider the action $A$ of $G^{[3]} \cap G(x)$ on $\Gamma(x)$. This action is the stabilizer in $L_{5}(2)$ of a 3 -subspace in the natural module, so that

$$
A \cong 2^{6}:\left(L_{3}(2) \times S_{3}\right)
$$

and $\mathrm{O}_{2}(\mathrm{~A})$ is the tensor product of the natural modules for $L_{3}(2)$ and $S_{3} \cong L_{2}(2)$ (in particular it is irreducible). Furthermore the action of $G_{0}^{[3]}$ 
on $\Sigma^{[4]}$ coincides with that of $O_{2}(A)$ and it is elementary abelian of order $2^{3}$. Thus $Q$ acts faithfully on the union of any two geometric subgraphs of valency 15 containing $\Sigma^{[3]}$ and the order of $Q$ is at most $2^{20}$. Since the action of $Q$ on $\Sigma^{[4]}$ is an irreducible $G_{-}^{[3]}$-module, it only remains to exclude the following two possibilities:

(a) $Q$ acts faithfully on $\Sigma^{[4]}$;

(b) the centralizer in $G_{+}^{[3]}$ of $G_{0}^{[3]} / K$ acts non-trivially on $Q$.

Suppose (a) holds and observe that a Sylow 3-subgroup of $G_{-}^{[3]}$ acts faithfully on $Q$. Since Aut $M_{22}$ acts on its Golay code module absolutely irreducibly, the possibility is excluded. Suppose that (b) holds. Then the action of $G_{+}^{[3]}$ on $Q$ contains $M_{22} \times M_{22}$ and it is easy to reach a contradiction using the fact that the action of $G_{+}^{[3]} \cap G(x)$ on $\Gamma(x)$ coincides with $O^{2,3}(A) \cong$ $2^{6}: L_{3}(2)$.

In order to identify the action of $G^{[3]}$ on the subgroup $N$ as in Lemma 10.8 consider a Sylow 3-subgroup $T$ of $G_{-}^{[3]}$. By Frattini argument

$$
G^{[3]}=N_{G^{[3]}}(T) G_{-}^{[3]},
$$

which shows that the image of $T$ in $L=G(x) / G_{1}(x)$ has 3-dimensional centralizer in a natural module.

LEMMA 10.9. The following assertions hold:

(i) $C_{G(x)}(T) / T \cong\left[2^{14}\right] \cdot L_{3}(2)$;

(ii) $\left|C_{G_{0}^{[3]}}(T)\right|=2^{10}$;

(iii) $C_{G_{0}^{[3]}}(T) \leq N$ and $\left[N: C_{G_{0}^{[3]}}(T)\right]=2^{2}$.

Proof. Since the centralizer of $T$ in the natural (or dual natural) module of $L$ is 3-dimensional, the centralizers of $T$ in $U^{[2]}$ and $U^{[3]}$ are 4-dimensional, so we have (i) by the structure of chief factors of $G(x)$. By the Frattini argument

$$
C_{G^{[3]}(x)}(T) G_{-}^{[3]}=G^{[3]}(x)
$$

and since $O_{2}\left(G^{[3]}(x)\right) / G_{0}^{[3]} \cong 2^{4}$, we have (ii). By Lemma $10.8 T$ acts fixedpoint freely on $Q$, so (iii) follows. 
Let us locate some characteristic subgroups of $G_{0}^{[3]}$.

LEMMA 10.10. Let $C^{[3]}, Z^{[3]}$ and $\Phi^{[3]}$ be the commutator subgroup, the centre and the Frattini subgroup of $G_{0}^{[3]}$, respectively. Then

(i) $G_{4}(x) \leq N$;

(ii) $C^{[3]} \leq N$;

(iii) $Z^{[3]} \leq N$ and $\left[N: Z^{[3]}\right] \geq 2^{3}$;

(iv) $\Phi^{[3]} \leq N$.

Proof. We follow notation introduced in Lemma 10.8. Since $G^{[3]} \cap G(x)$ does not stabilize hyperplanes in the dual natural module $G_{4}(x)$ of $L$, $G_{4}(x)$ is contained in $G_{0}^{[3]}$ by Lemma 10.3 (iii). Consider the action of $A=2^{6}:\left(S_{3} \times L_{3}(2)\right)$ on $G_{4}(x)$. Since $A$ is the stabilizer in $L$ of a 3 -subspace in the natural module, $T G_{1}(x) / G_{1}(x)$ is a Sylow 3-subgroup of $O_{2,3}(A)$. Furthermore, $G_{4}(x)=W_{1} \oplus W_{2}$, where $W_{1}=C_{G_{4}(x)}(T)$ is 3-dimensional, $W_{2}=\left[G_{4}(x), T\right]$ is 2-dimensional and also $W_{2}=\left[O_{2}(A), G_{4}(x)\right]$. By Lemma 10.9 (ii) $W_{1} \leq N$. Since $Q$ is abelian and

$$
G_{0}^{[3]} G_{1}(x) / G_{1}(x)=O_{2}(A),
$$

$W_{2} \leq N$ as well and (i) follows. Since $Q$ is elementary abelian and $G^{[3]}$ acts on $Q$ irreducibly (ii) to (iv) follow from (i).

Let $X$ be the image of $G^{[3]}$ in the outer automorphism group of $N$. Since every 2 -automorphism of the 2-group $G_{0}^{[3]}$ acts faithfully on the Frattini factor, by Lemmas 10.8 and 10.10 (iii) $X$ contains a normal subgroup $Y$ such that

$$
X / Y \cong \operatorname{Aut} M_{22} \times S_{3}
$$

LEMMA 10.11. The group $N$ contains exactly two chief factors of $G^{[3]}$. One of the factors is a 10-dimensional irreducible module of Aut $M_{22}$ and the other one is a 2-dimensional natural module of $L_{2}(2) \cong S_{3}$.

Proof. Recall that $|N|=2^{12}$. By [Ja73] an irreducible faithful $G F(2)$ representation of Aut $M_{22}$ of dimension less than or equal to 12 is 10dimensional representation on the Golay code module or on the Todd module. Furthermore these modules are absolutely irreducible. This implies the statement. 
We follow notations introduced in Lemma 10.10.

LEMMA 10.12. The following assertions hold:

(i) $Z^{[3]}$ is of order $2^{2}$ and $G_{-}^{[3]} / G_{0}^{[3]} \cong S_{3}$ acts faithfully on $Z^{[3]}$, particularly $Z\left(G_{-}^{[3]}\right)$ is trivial;

(ii) $N$ is elementary abelian;

(iii) $C^{[3]}=\Phi^{[3]}=N$.

Proof. The assertion (i) follows from Lemmas 10.10 (iii) and 10.11. By (i) and Lemma $10.11 G^{[3]}$ induces on $N / Z^{[3]}$ an irreducible action of Aut $M_{22}$ on the Golay code Module or on the Todd module (which are absolutely irreducible). None of these two modules is self-dual (they are dual to each other), which gives (ii). By (i) $G_{0}^{[3]}$ induces a non-trivial action on $N$ and since $G^{[3]}$ acts irreducibly on $Q=G_{0}^{[3]} / N$ and $N$ is abelian, the action is isomorphic to $Q$. By (ii) and the order reason this action coincides with that of $\mathrm{O}_{2}(J)$ where

$$
J \cong 2^{20}:\left(S_{3} \times L_{10}(2)\right)
$$

is the stabilizer of $Z^{[3]}$ in the general linear group $L_{12}(2)$ of $N$. This immediately gives (iii).

As a consequence of the proof of Lemma 10.12 we obtain the following.

Lemma 10.13. The action induced by $G_{0}^{[3]}$ on $N$ is isomorphic to $Q$ and it is generated by the transvections whose axis contain $Z^{[3]}$ and whose centre are contained in $Z^{[3]}$. In particular the module in Lemma 10.11 is the Todd module for Aut $M_{22}$.

Next we discuss automorphisms of $G_{-}^{[3]}$ trivial on $G_{0}^{[3]}$.

LEMmA 10.14. Let $Y=C_{\text {Aut } G_{-}^{[3]}}\left(G_{0}^{[3]}\right)$ and $X$ be the group of inner automorphisms of $G_{-}^{[3]}$ induced by $Z^{[3]}$. Then $Y=X$.

Proof. Since a non-identity element of $Z^{[3]}$ is in the centre of $G_{0}^{[3]}$, but not in the centre of $G_{-}^{[3]}, X \cong 2^{2}$ is a subgroup of $Y$. Let $y \in Y$ and $T$ be a Sylow 3 -subgroup in $G^{[3]}$ and suppose that $T^{y} \neq T$. Since $y$ commutes with 
$G_{0}^{[3]}$ and $Z^{[3]}$ is the centre of $G_{0}^{[3]}, T Z^{[3]}=T^{y} Z^{[3]}$. Since $T$ acts fixed-point freely on $Z^{[3]}$, there is $x \in X$, such that $T^{x}=T^{y}$ and trading $y$ by $y x$, we can assume that $y$ centralizes $T$. Then $y$ normalizes $M:=N_{N}(T)$. By Lemma $10.9 M$ is of order $2^{11}$ and by the Frattini argument in view of Lemma $10.13 M \cap N$ is elementary abelian of order $2^{10}$, on which $N_{G^{[3]}}(T)$ induces an action of Aut $M_{22}$ as on the Todd module. Since the action is irreducible, it is easy to see that the whole $M$ is elementary abelian. Thus for every $\tau \in M \backslash N$ we have $\langle T, \tau\rangle \cong S_{3}$ is a complement to $G_{0}^{[3]}$ in $G_{-}^{[3]}$ containing $T$ and $Y / X$ acts faithfully on the set of such complements. Since $M \cap Z\left(G_{0}^{[2]}\right)=1$, the action of the complements is in fact trivial. Hence the result.

As above let $I^{[3]}$ be the image of $G^{[3]}$ in Out $G_{-}^{[3]}$ (since the centre of $G_{-}^{[3]}$ is trivial by Lemma $\left.10.12(\mathrm{i}), I^{[3]}=G^{[3]} / G_{-}^{[3]}\right)$. The results proved in this subsection can be summarized as follows.

Proposition 10.15. The group $I^{[3]}$ contains a normal subgroup $Y^{[3]}$, such that

(i) every element of $Y^{[3]}$ is the image of an automorphism of $G_{-}^{[3]}$ which centralizes both $N$ and $Q$ but acts non-trivially on $G_{0}^{[3]}$;

(ii) is a 2-group;

(iii) $I^{[3]} / Y^{[3]} \cong$ Aut $M_{22}$.

Proof. By Lemma 10.14 every element of $I^{[3]}$ is the image of an automorphism acting non-trivially on $G_{0}^{[3]}$. By Lemma 10.11 the kernel of the action on $Q$ contains the kernel of the action on $N$ and the image in $I^{[3]}$ of the latter action is Aut $M_{22}$. Since (ii) is by Lemma 10.10 (iv), the result follows.

In the next subsection we will show that $Y^{[3]}$ is in fact trivial.

10.3. The action of $G^{[4]}$ on $G_{-}^{[4]}$

First we identify $G_{-}^{[4]}$ as a subgroup of $G_{-}^{[3]}$ and summarise what we know so far about the action of $G^{[3]} \cap G^{[4]}$ on $G_{-}^{[4]}$ (we assume that the hypothesis of Lemma 10.14 holds).

LEMMA 10.16. The following assertions hold:

(i) $\left|G_{-}^{[4]} G_{0}^{[3]} / G_{0}^{[3]}\right|=2$ and $Z^{[4]}:=C_{Z^{[3]}}\left(G_{-}^{[4]}\right)$ is of order 2 ; 
(ii) $N \leq G_{-}^{[4]}$;

(iii) $\left(G_{-}^{[4]} \cap G_{0}^{[3]}\right) / N$ is a 10-dimensional submodule in $Q$ invariant under $G_{+}^{[3]} / C_{G_{+}^{[3]}}(Q) \cong \operatorname{Aut} M_{22}$

(iv) $G_{-}^{[4]} \cap G_{0}^{[3]}$ induces on $N$ an action of order $2^{10}$ generated by the transvections with the centre $Z^{[4]}$ and with axises containing $Z^{[3]}$;

(v) $Z^{[4]}$ is the centre of $G_{-}^{[4]}$;

(vi) $Z^{[4]} \leq G_{4}(x)$.

Proof. (i) follows from Lemmas 10.3 (iii) and 10.12 (i). Since $N$ is the vertex-wise stabilizer in $G_{0}^{[3]}$ of the geometric subgraphs of valency 15 containing $\Sigma^{[3]}$, (ii) follows. By Lemma $10.7 Q$ induces an action of order $2^{10}$ on $\Sigma^{[4]}$, so (iii) is by Lemma 10.8. Since $G_{-}^{[3]} / G_{0}^{[3]} \cong S_{3}$ acts faithfully on the set of subgroups of order 2 in $\Sigma^{[3]}$ and on the set of $G_{+}^{[3]}$-invariant submodules in $Q$, (iv) follows from Lemma 10.13. Now (v) is immediate from (i) to (iv) and (vi) is by (v) and Lemma 10.2.

LEMMA 10.17. A proper subgroup of $G_{-}^{[4]}$ normalized by the action of $G^{[3]} \cap G^{[4]}$ contains $Z^{[4]}$ and it is one of the following four subgroups:

$$
Z^{[4]}, Z^{[3]}, \quad N \text { and } G_{-}^{[4]} \cap G_{0}^{[3]},
$$

whose orders are $2,2^{2}, 2^{12}$ and $2^{22}$, respectively.

Proof. This follows from Lemma 10.15 and Proposition 10.14.

LEMMA 10.18. The following assertions hold:

(i) $G_{-}^{[4]} / Z^{[4]}$ is an elementary abelian 2-group on which $G^{[4]}$ acts irreducibly;

(ii) $G_{-}^{[4]} \cong 2_{+}^{1+22}$.

Proof. Let $P$ be a proper subgroup in $G_{-}^{[4]}$ normalized by $G^{[4]}$. Then $P$ is one of the four subgroups in Lemma 10.16, in particular $P$ contains $Z^{[4]}$. On the other hand, $P$ must be normalized by $G(x) \cap G^{[4]}$ and by Lemma 10.2 (iii) the latter has exactly six chief factors inside $G_{-}^{[4]}$, four of them are 4-dimensional, one 6-dimensional and one 1-dimensional. By Lemma $10.15(\mathrm{v})$ the latter factor is in $Z^{[4]}$ and by above it must be in $P$. 
Then the non-existence of $P$ follows already from the order consideration. Thus there is a unique chief factor of $G^{[4]}$ inside $G_{-}^{[4]} / Z^{[4]}$, which gives (i) and the fact that $G_{-}^{[4]}$ is extraspecial. Since $N$ is an elementary abelian subgroup in $G_{-}^{[4]}$ or order $2^{12}$, (ii) follows.

The subgroup $Z^{[3]}$ is elementary abelian subgroup of order $2^{2}$ in $G_{-}^{[4]}$ which contains the centre of $G_{-}^{[4]}$ and $G_{-}^{[4]} \cap G_{0}^{[3]}$ coincides with $C_{G_{-}^{[4]}}\left(Z^{[4]}\right)$ and also with $C_{G_{-}^{[4]}}\left(Z^{a}\right)$, where $Z^{a}$ is a subgroup of order 2 in $Z^{[3]}$ other than $Z^{[4]}$. Then

$$
E:=\left(G^{[4]} \cap G_{0}^{[3]}\right) / Z^{a}
$$

is an extraspecial group of type $2_{+}^{1+20}$, whose outer automorphism group $F$ is isomorphic to $O_{20}^{+}(2)$. Let us determine the image $B$ of $G^{[3]} \cap G^{[4]}$ in $F$. By Lemmas 10.15 and 10.16 (iv) $B$ contains a normal 2-subgroup $Y$, such that

$$
\bar{B}:=B / Y \cong 2^{10} \text {. Aut } M_{22}
$$

and $\mathrm{O}_{2}(\bar{B})$ is the Golay code module for $\bar{B} / O_{2}(\bar{B})$. On the other hand, $N / Z^{a}$ is a maximal abelian subgroup (of order $2^{11}$ ) in $E$. Let $C$ be the stabilizer of $N / Z^{a}$ in $F$. Then, since $W:=N / Z^{[3]}$ is a maximal totally singular subspace in the natural module $E / Z^{[4]}$ of $F, C$ is the semidirect product of the exterior square $W^{[2]}$ of $W$ and the linear group $L_{10}(2)$ of $W$ (compare Section 6). Thus $O_{2}(B)$ (which is an extension of the unknown 2-group $Y$ by the Golay code module) is a subspace in $W^{[2]}$ invariant under Aut $M_{22} \leq L_{10}(2)$.

LEMmA 10.19. Let $W$ be the 10-dimensional Todd module for Aut $M_{22}$. Then $W^{[2]}$ possesses a unique composition series

$$
0<W_{1}^{[2]}<W_{2}^{[2]}<W^{[2]}
$$

where $W_{1}^{[2]}$ is the Golay code module, $\operatorname{dim}\left(W_{2}^{[2]} / W_{1}^{[2]}\right)=1$ and $\operatorname{dim}\left(W^{[2]} /\right.$ $\left.W_{2}^{[2]}\right)=34$.

Proof. The composition factors of $W^{[2]}$ can be calculated from [Ja73] by decomposing the Brauer character. The exact submodule structure of $W^{[2]}$ was established using GAP [GAP] by Dima Pasechnik, to whom we are very thankful. 
By Lemma 10.19 and the paragraph before that lemma we have the following.

Corollary 10.20. The image $B$ of $G^{[3]} \cap G^{[4]}$ in Out $E$ is isomorphic to $2^{10}$. Aut $M_{22}$ (an extension of the Golay code module by Aut $M_{22}$ ).

Notice that $O_{2}(B)$ is the image of $G_{0}^{[3]}$ in $F=$ Out $E$ (compare Lemma 10.16 (iii)). Now we are ready to show that the subgroup $Y^{[3]}$ in Proposition 10.15 is trivial.

Proposition 10.21. The image $I^{[3]}$ of $G^{[3]}$ in Out $G_{-}^{[3]}$ is isomorphic to Aut $M_{22}$.

Proof. Let $y \in G^{[3]}$ and suppose that the automorphism of $G_{-}^{[3]}$ induced by $y$ maps onto the subgroup $Y^{[3]}$ in Out $G_{-}^{[3]}$ as in Proposition 10.15. Then $y$ acts trivially on both $N$ and $Q$ and by the proof of Lemma 10.14 we can adjust $y$ by the inner automorphism induced by an element of $Z^{[3]}$, so that $y$ centralizes $T$. By Lemma 10.20 we can further adjust $y$ to act trivially on $\left(G^{[4]} \cap G_{0}^{[3]}\right) / Z^{a}$ and since there are two choices for $Z^{a}$, we can adjust $y$ to centralize the whole $G_{-}^{[4]} \cap G_{0}^{[3]}$. Finally, instead of $\Sigma^{[4]}$ we could take another geometric subgraph of valency 15 containing $\Sigma^{[3]}$ (which is the image of $\Sigma^{[4]}$ under some $\left.t \in T^{\#}\right)$. Since $y$ commutes with $T$, it must centralize $\left(G_{-}^{[4]}\right)^{t} \cap G_{0}^{[3]}$ as well and hence it centralizes the whole

$$
G_{0}^{[3]}=\left\langle G_{-}^{[4]} \cap G_{0}^{[3]},\left(G_{-}^{[4]}\right)^{t} \cap G_{0}^{[3]}\right\rangle
$$

and the image of $y$ in Out $G_{-}^{[3]}$ is trivial.

Corollary 10.22. The image $I^{[4]}$ of $G^{[4]}$ on Out $G_{-}^{[4]}$ is isomorphic to $\mathrm{Co}_{2}$.

Proof. The homomorphism

$$
\varphi^{[4]}: G^{[4]} \longrightarrow \text { Out } G_{-}^{[4]}
$$

induces a faithful completion of the amalgam

$$
\mathcal{A}^{[4]} \cong \mathcal{A}_{4}^{(3)} \cong \mathcal{A}\left(\mathrm{Co}_{2}, \Gamma\left(\mathcal{G}\left(\mathrm{Co}_{2}\right)\right)\right.
$$

and by Lemma 10.20 and Proposition 10.21 the image of $G^{[3]} \cap G^{[4]}$ under $\varphi^{[4]}$ is $2^{10}$. Aut $M_{22}$. Now the result is by Proposition 2.4 . 


\subsection{Proof of Proposition $\mathbf{1 0 . 5}$}

The structures of $Z\left(G_{-}^{[3]}\right)$ and $Z\left(G_{-}^{[4]}\right)$ were established in Lemmas 10.12 (i) and $10.16(\mathrm{v})$, while the images of $G^{[3]}$ and $G^{[4]}$ in Out $G_{-}^{[3]}$ and Out $G_{-}^{[4]}$ were specified in Proposition 10.21 and Corollary 10.22, respectively. So in order to complete the proof of Proposition 10.5 it only remains to consider the case $i=2$.

Lemma 10.23. The following assertions hold:

(i) the centre $Z^{[2]}$ of $G_{0}^{[2]}$ is elementary abelian of order $2^{3}$ contained in $G_{4}(x)$

(ii) $G_{-}^{[2]} / G_{0}^{[2]} \cong L_{3}(2)$ acts faithfully on $Z^{[2]}$ and $Z\left(G_{-}^{[2]}\right)=1$.

Proof. Let $\mathcal{S}=\left\{{ }^{i} \Sigma^{[4]} \mid 1 \leq i \leq 15\right\}$ be the set of geometric subgraphs of valency 15 containing $\Sigma^{[2]}$. Then $\mathcal{S}$ carries the structure of the point-set of the projective plane of order 2 . The vertex-wise stabilizer ${ }^{i} G_{-}^{[4]} \cong 2_{+}^{1+22}$ of ${ }^{i} \Sigma^{[4]}$ (cf. Lemma 10.18) is contained in $G_{-}^{[2]}$. If ${ }^{i} Z^{[4]}=Z\left({ }^{i} G_{-}^{[4]}\right)$, then by Lemma 10.16 (vi) ${ }^{i} Z^{[4]}$ is a 1 -subspace in $G_{4}(x) \cong U^{*}$, therefore

$$
Z^{[2]}:=\left\langle{ }^{i} Z^{[4]} \mid 1 \leq i \leq 15\right\rangle
$$

is a 3-subspace in $G_{4}(x)$ and $G_{0}^{[2]}$ is contained in $C_{G_{-}^{[2]}}\left(Z^{[2]}\right)$. Since $G_{-}^{[4]}$ is extraspecial,

$$
Z\left(C_{G_{-}^{[4]}}\left(Z^{[2]}\right)\right)=Z^{[2]}
$$

and (i) follows. Now (ii) is immediate from the action of $G_{-}^{[2]} G_{1}(x) / G_{1}(x)$ on $G_{4}(x) \cong U^{*}$.

For $i=2,3$ and 4 put

$$
\bar{G}^{[i]}=G^{[i]} / C_{G^{[i]}}\left(G_{-}^{[i]}\right)
$$

and let $\bar{\varphi}^{[i]}$ be the natural homomorphism of $G^{[i]}$ onto $\bar{G}^{[i]}$, so that $\varphi^{[i]}$ is the composition of $\bar{\varphi}^{[i]}$ together with the factoring out the inner automorphisms of $G_{-}^{[i]}$. Then the results, established in Subsections 10.2 and 10.3 imply that

$$
\left.\bar{G}^{[3]} \cong 2^{2+10+20} \text {. Aut } M_{22} \times S_{3}\right) \text { and } \bar{G}^{[4]} \cong 2^{22} \cdot \mathrm{Co}_{2} .
$$

Lemma 10.24. For $2 \leq i<j \leq 4$ let $K_{j}^{[i]}$ and $K_{i}^{[j]}$ be the kernels of the restrictions to $G^{[2]} \cap G^{[3]}$ of $\bar{\varphi}^{[i]}$ and $\bar{\varphi}^{[j]}$, respectively. Then 
(i) $K_{3}^{[2]}=K_{2}^{[3]}$;

(ii) $\left[K_{i}^{[4]}: K_{4}^{[i]}\right]=2$ and $K_{i}^{[4]}=K_{4}^{[i]} Z^{[4]}$.

Proof. Since $G_{-}^{[i]} \leq G_{-}^{[j]}$, it is clear that $K_{j}^{[i]} \leq K_{i}^{[j]}$. By the paragraph before the lemma we have

$$
J:=\bar{\varphi}^{[3]}\left(G^{[2]} \cap G^{[3]}\right) \cong 2^{2+10+20} \cdot\left(2^{5}: S_{5} \times S_{3}\right)
$$

(here $2^{5}: S_{5}$ is the stabilizer in Aut $M_{22}$ of an element of the right-most type in the Petersen geometry $\left.\mathcal{G}\left(M_{22}\right)\right)$. Furthermore, if $S \cong 2^{2+10+20} \cdot\left(2^{5} \times S_{3}\right)$ is the largest solvable normal subgroup in $J$ then

$$
S=\bar{\varphi}^{[3]}\left(G_{-}^{[2]} \cap G^{[3]}\right) \cong G_{-}^{[2]} \cap G^{[3]} .
$$

Therefore $K_{2}^{[3]}$ and $G_{-}^{[2]} \cap G^{[3]}$ are two normal subgroups in $G^{[2]} \cap G^{[3]}$ with trivial intersection. Hence

$$
K_{2}^{[3]} \leq C_{G^{[2]} \cap G^{[3]}}\left(G_{-}^{[2]} \cap G^{[3]}\right) .
$$

In order to show that the latter coincides with $K_{3}^{[2]}$ is it sufficient to establish that every automorphism of $G^{[2]}$ which centralizes $G_{-}^{[2]} \cap G^{[3]}$, centralizes the whole $G_{-}^{[2]}$. Let $y \in C_{G^{[3]}}\left(G_{-}^{[2]} \cap G^{[3]}\right)$. Then $y$ commutes with $G_{0}^{[2]}$. Let $J$ be a Sylow 7-subgroup in $G_{-}^{[2]}$. Since $J$ and $G_{-}^{[2]} \cap G^{[3]}$ generate $G_{-}^{[2]}$, all we have to show is that $y$ centralizes $J$. Let $V=O^{2}\left(N_{G_{-}^{[2]}}(J)\right) \cong F_{7}^{3}$. Then by Frattini argument we can assume that a Sylow 3-subgroup $S$ in $V$ is contained in $G_{-}^{[2]} \cap G^{[3]}$. Since $y$ commutes with $G_{0}^{[2]}$ we have $V^{y}=V^{z}$ for some $z \in Z^{[4]}=Z\left(G_{0}^{[2]}\right)$ (the latter equality is by Lemma 10.23). Since $y$ commutes with $S, z$ is contained in $C_{Z^{[4]}}(S)$, which is of order 2 . Since instead of $S$ we can take a subgroup of order 3 in $G_{-}^{[2]} \cap G^{[3]}$ which does not centralize $z$ (clearly such a subgroup exists), $y$ must commute with $J$, hence (i) follows. The assertion (ii) can be proved similarly with the only difference that the centre $Z^{[4]}$ of $G_{-}^{[4]}$ is of order 2 .

It is easy to deduce from Lemma 10.23 that the images under $\varphi^{[2]}$ of the subgroups

$$
G^{[2]} \cap G^{[3]}, G^{[2]} \cap G^{[4]} \text { and } G^{[2]} \cap G^{[3]} \cap G^{[4]}
$$


are the same subgroup of Out $G_{-}^{[2]}$, isomorphic to $S_{5}$. Since $G^{[2]}$ is clearly generated by its intersections with $G^{[3]}$ and $G^{[4]}$, we have

$$
I^{[2]}:=\varphi^{[2]}\left(G^{[2]}\right) \cong S_{5},
$$

which completes the proof of Proposition 10.5.

\subsection{The rank 5 amalgam}

Now with a group $G$, whose locally projective action of a graph $\Gamma$ corresponds to case (vi) in Proposition 4.1 we associate an amalgam, which 'resembles' the amalgam of maximal parabolics associated with the action of the Baby Monster group on its P-geometry $\mathcal{G}(B M)$ (cf. Lemma 10.4).

Since $G_{-}^{[i]} \neq 1$ for $2 \leq i \leq 4, \Gamma$ contains a complete family of geometric subgraphs by Lemma 2.2 , so we can start with the amalgam

$$
\mathcal{F}=\left\{G^{[i]} \mid 0 \leq i \leq 4\right\}
$$

of maximal parabolics associated with the action of $G$ on $\mathcal{G}(G, \Gamma)$ and construct a suitable quotient

$$
\widehat{\mathcal{F}}=\left\{\widehat{G}^{[i]} \mid 0 \leq i \leq 4\right\}
$$

of $\mathcal{F}$. It is quite clear that we should set $\widehat{G}^{[i]}=G^{[i]}$ for $i=0$ and 1 and

$$
\widehat{G}^{[i]}=\bar{G}^{[i]}:=G^{[i]} / C_{G^{[i]}}\left(G_{-}^{[i]}\right)
$$

for $i=2$ and 3. Since the restrictions of $\bar{\varphi}^{[2]}$ and $\bar{\varphi}^{[3]}$ to $G^{[i]} \cap G^{[2]}$ and $G^{[i]} \cap$ $G^{[3]}$, are isomorphisms for $i=0$ and 1 and their restrictions to $G^{[2]} \cap G^{[3]}$ coincide by Lemma 10.24 (i), there is a homomorphism $\widehat{\varphi}$ of the amalgam $\mathcal{E}=\left\{G^{[i]} \mid 0 \leq i \leq 3\right\}$ onto $\widehat{\mathcal{E}}=\left\{\widehat{G}^{[i]} \mid 0 \leq i \leq 3\right\}$. Namely, $\widehat{\varphi}$ is the identity map when restricted to $G^{[0]} \cup G^{[1]}$, while restricted to $G^{[2]}$ and $G^{[3]}$ it coincides with $\bar{\varphi}^{[2]}$ and $\bar{\varphi}^{[3]}$, respectively. We adopt the hat convention for the subgroups contained in $\mathcal{E}$.

Notice that we can not just define $\widehat{G}^{[4]}$ to be $\bar{G}^{[4]}$, since the kernel of $\bar{\varphi}^{[4]}$ contains $Z^{[4]}$, while $\widehat{Z}^{[4]} \cong Z^{[2]}$ is of order 2 . Instead we define $\widehat{G}^{[4]}$ to be the universal completion of the subamalgam

$$
\widehat{\mathcal{C}}=\left\{\widehat{C}^{[i]} \mid 0 \leq i \leq 3\right\}
$$

in $\widehat{\mathcal{E}}$, where $C^{[i]}=G^{[i]} \cap G^{[4]}$, or equivalently $\widehat{C}^{[i]}=C_{\widehat{G}^{[i]}}\left(\widehat{Z}^{[4]}\right)$.

LEMMA 10.25. One of the following two possibilities occurs: 
(i) $\widehat{G}^{[4]} \cong 2_{+}^{1+22} \cdot$ Co $_{2}$ and the homomorphism $\widehat{\varphi}$ extends to a homomorphism of $\mathcal{F}$ onto $\widehat{\mathcal{F}}$;

(ii) $\widehat{G}^{[4]} \cong \bar{G}^{[4]} \cong 2^{22}$.Co 2 and $\widehat{\mathcal{C}}$ does not possess faithful completions.

Proof. If we set $\bar{C}^{[i]}=\widehat{C}^{[i]} / \widehat{Z}^{[4]}$, then $\overline{\mathcal{C}}:=\left\{\bar{C}^{[i]} \mid 0 \leq i \leq 3\right\}$ is the amalgam formed by the images of the subgroups $C^{[i]}$ in the automorphism group of $G_{-}^{[4]} \cong 2_{+}^{1+22}$. By Corollary 10.22 these images generate $\bar{G}^{[4]} \cong 2^{22} . \mathrm{Co}_{2}$, so that modulo the group of inner automorphisms (which is elementary abelian of order $2^{22}$ ) they generate the group $\mathrm{Co}_{2}$. Furthermore,

$$
\left\{\widehat{C}^{[i]} / \widehat{G}_{-}^{[4]} \mid 0 \leq i \leq 3\right\}
$$

is the amalgam of maximal parabolics associated with the action of $\mathrm{Co}_{2}$ on its $\mathrm{P}$-geometry $\mathcal{G}\left(\mathrm{Co}_{2}\right)$. Since the latter geometry is simply connected (cf. [Iv99]), $\bar{G}^{[4]}$ is the universal completion of $\overline{\mathcal{C}}$. Clearly there is a homomorphism of $\widehat{\mathcal{C}}$ onto $\overline{\mathcal{C}}$ with kernel $\widehat{Z}^{[4]}$. Therefore either $\bar{G}^{[4]}$ is also the universal completion of $\widehat{\mathcal{C}}$ and (ii) holds or the universal completion of $\widehat{\mathcal{C}}$ is faithful and (i) holds.

LEMMA 10.26. The amalgam $\overline{\mathcal{C}}$ in the proof of Lemma 10.25 is determined uniquely up to isomorphism.

Proof. First, $\bar{G}^{[4]}$ is determined uniquely up to isomorphism as the preimage in the automorphism group of $G_{-}^{[4]} \cong 2_{+}^{1+22}$ of the $\mathrm{Co}_{2}$-subgroup in $O_{22}^{+}(2) \cong$ Out $G_{-}^{[4]}$. Second, $\overline{\mathcal{C}}$ is the preimage in $\bar{G}^{[4]}$ of the amalgam of maximal parabolics of the action of $\bar{G}^{[4]} / \mathrm{O}_{2}\left(\bar{G}^{[4]}\right) \cong \mathrm{Co}_{2}$ on $\mathcal{G}\left(\mathrm{Co}_{2}\right)$, hence the result.

In a sense $\widehat{\mathcal{C}}$ is an extension of $\overline{\mathcal{C}}$ over a centre of order 2 . If $\widetilde{G}$ is the Baby Monster, then

$$
\widetilde{\mathcal{C}}=\left\{\widetilde{G}^{[i]} \cap \widetilde{G}^{[4]} \mid 0 \leq i \leq 3\right\}
$$

is such an extension and clearly $\widetilde{G}^{[4]} \cong 2_{+}^{1+22} \cdot C_{2}$ is the faithful universal completion of $\widetilde{\mathcal{C}}$. We will exclude the possibility (ii) in Lemma 10.25 by showing that $\widehat{\mathcal{C}}$ is isomorphic to $\widetilde{\mathcal{C}}$. Of course in order to accomplish this it is sufficient to prove that $\widehat{\mathcal{C}}$ is determined uniquely up to isomorphism.

Lemma 10.27 . Let $I=\widehat{G}_{+}^{[3]} / \widehat{Z}^{[3]} \cong 2^{10+2 \cdot 10}$. Aut $M_{22}$ and $B=$ Out $I$. Then 
(i) I is determined uniquely up to isomorphism;

(ii) I contains three normal elementary abelian subgroups of order $2^{20}$ and four classes of complements to $\mathrm{O}_{2}(I)$;

(iii) $B$ acts faithfully on the four classes of complements and $S_{3} \leq B$;

(iv) $\widehat{G}^{[3]} / \widehat{Z}^{[3]}$ is determined uniquely up to isomorphism;

(v) $\widehat{G}_{+}^{[3]}$ is determined uniquely up to isomorphism;

(vi) $\widehat{G}^{[3]}$ is determined uniquely up to isomorphism.

Proof. Since $I$ is a uniquely defined section in $\widehat{G}_{+}^{[3]} / \widehat{Z}^{[4]}$, (i) follows from Lemma 10.26. By Lemmas 10.8, 10.16, $10.18 I$ contains exactly three normal elementary abelian subgroups of order $2^{20}$, each containing two chef factors of $I$, isomorphic to the Golay code module and the Todd module of $I / O_{2}(I) \cong$ Aut $M_{22}$. If $T \in S_{y} l_{3}\left(\widehat{G}_{-}^{[3]}\right)$, then by Lemmas $10.8,10.13$ we have $F:=C_{\widehat{G}_{+}^{[3]}}(T) \cong 2^{10}$. Aut $M_{22}$ and $O_{2}(F)$ is the Todd module. Since the latter has trivial second cohomology (cf. Table VI on p. 167 in [IS02]), F splits over its $\mathrm{O}_{2}$ and hence so does $I$. Since the first cohomology is trivial for the Todd module and 1-dimensional for the Golay code module, $\widehat{G}_{+}^{[3]} / \widehat{N}$ has four classes of complements. Since every extension by Aut $M_{22}$ of the Todd module splits, they lead to four classes of complements in $I$, so we have (ii).

Let $M_{0} \cong$ Aut $M_{22}$ be a complement to $O_{2}(I)$ in $I$ and $J_{1}, J_{2}, J_{3}$ be the normal elementary abelian subgroups of order $2^{20}$ in $I$. Then by the proof of (ii) for $k=1,2,3$ the subgroup $J_{k} M_{0}$ contains two classes of complements to $J_{k}$ and if $M_{k}$ is a complement not in the class of $M_{0}$, then $\left\{M_{0}, M_{1}, M_{2}, M_{3}\right\}$ is a complete set of representatives of the complements to $\mathrm{O}_{2}(I)$ in $I$.

Let $y$ be an automorphism of $I$ which stabilizes every class of complements to $\mathrm{O}_{2}(I)$. Then by the above $y$ normalizes $J_{k}$ for $k=1,2$ and 3 and, adjusted by a suitable inner automorphism, it centralizes $M_{0}$. Since $J_{k}$ contains two irreducible $M_{0}$-factors which are not isomorphic, $y$ must centralize $J_{k}$, which gives the first assertion in (iii). Since $I$ is isomorphic to a section in the parabolic subgroup $\widetilde{G}^{[3]}$ in the Baby Monster, it is clear that $B$ contains $S_{3}$, which gives the second assertion in (iii).

Since the centralizer of $I$ in $\widehat{G}^{[3]} / \widehat{Z}^{[3]}$ is trivial, the latter can be identified with its image in the automorphism group of $I$ and by (iii) this image is determined up to conjugation in Aut $I$, hence (iv) follows. 
Turning to $(\mathrm{v})$, observe that $\widehat{G}_{+}^{[3]}$ is the elementary abelian group $\widehat{Z}^{[3]}$ extended by the group $I$ (which is known by (i)) and that $\widehat{G}^{[3]}$ induces the outer automorphism groups of both $I$ and $\widehat{Z}^{[3]}$. In addition, $\widehat{G}_{+}^{[3]} / \widehat{Z}^{[4]}$ (whose isomorphism type is known by Lemma 10.26) is a quotient of $\widehat{G}^{[3]}$ over a subgroup of order 2 in $\widehat{Z}^{[3]}$ and $\widehat{G}_{+}^{[3]} / \widehat{Z}^{[4]}$ does not split over $\widehat{Z}^{[3]} / \widehat{Z}^{[4]}$. Let $N$ be the largest non-split central extension by $I=\widehat{G}_{+}^{[3]} / \widehat{Z}^{[3]}$ of an elementary abelian 2-group $E$. Let $E_{0}$ be the kernel of the natural homomorphism of $N$ onto $\widehat{G}_{+}^{[3]} / \widehat{Z}^{[4]}$. By the above $E_{0}$ is a hyperplane in $E$. Let $C$ be the image of $\widehat{G}^{[3]} / \widehat{Z}^{[3]}$ in $B$ under the natural homomorphism. Then by (iii) $C$ is uniquely determined up to conjugation and by the universality property $C$ acts on $E$. Consider the images of $E_{0}$ under this action. Since $\widehat{G}_{+}^{[3]}$ has index 2 in its normalizer in $\widehat{G}^{[3]}$, there are at most three different images. If $F$ is the intersection of these images, then $E / F$ is of order $2^{2}$ or $2^{3}$ and if $F^{*}$ is the preimage in $E$ of the centralizer in $E / F$ of an element of order 3 in $C$, then

$$
\widehat{G}_{+}^{[3]} \cong E / F^{*}
$$

and (v) follows.

By Lemma 10.14 (ii) $\widehat{G}^{[3]}$ is the semidirect product of $\widehat{G}_{+}^{[3]}$ and $R \cong S_{3}$. The images of $R$ in the automorphism groups of $I$ and $\widehat{Z}^{[3]}$ are determined up to conjugation. Hence two different images of $R$ in the automorphism group of $\widehat{G}_{+}^{[3]}$ (modulo inner automorphisms) differ by automorphisms which act trivially on $O^{2}\left(\widehat{G}_{+}^{[3]}\right)$. By (ii) and Frattini argument (notice that a Sylow 3 -subgroup of $\widehat{G}_{+}^{[3]}$ acts fixed-point freely on $\left.\widehat{Z}^{[3]}\right)$, there is a complement $M \cong$ Aut $M_{22}$ to $\widehat{G}_{0}^{[3]}$ in $\widehat{G}_{+}^{[3]}$. Since $\widehat{Z}^{[3]} M \cong 2^{2} \times$ Aut $M_{22}$, the group of automorphisms of $\widehat{G}_{+}^{[3]}$ acting trivially on $O^{2}\left(\widehat{G}_{+}^{[3]}\right)$ is elementary abelian of order $2^{2}$ and $\widehat{G}^{[3]}$ induces on the latter the fixed-point free action of $S_{3}$. Since all $S_{3}$-subgroups in $S_{4}$ are conjugate, we obtain (vi).

LEMMA 10.28. The following assertions hold:

(i) the amalgams $\widehat{\mathcal{C}}$ and $\widetilde{\mathcal{C}}$ are isomorphic;

(ii) the possibility (i) in Lemma 10.25 holds.

Proof. Let $\widehat{S}$ be a Sylow 2-subgroup in $\widehat{G}^{[3]}$. By Lemma 10.27 (vi) the isomorphism type of $\widehat{S}$ is uniquely determined. Furthermore, we can choose 
$\widehat{S}$ so that for every $J \subset\{0,1,2,3\}$ and

$$
\widehat{\mathcal{C}}^{[J]}:=\bigcap_{i \in J} \widehat{\mathcal{C}}^{[i]}
$$

the intersection $\widehat{\mathcal{C}}^{[J]} \cap \widehat{S}$ is a Sylow 2-subgroup of $\widehat{\mathcal{C}}^{[J]}$. Now (i) follows from Gaschütz' theorem. Since $\widehat{\mathcal{C}}$ is determined uniquely up to isomorphism (ii) follows by the paragraph before Lemma 10.27 .

By Lemmas 10.24, 10.25 and 10.27 the amalgam $\widehat{\mathcal{F}}$ is of $B M$-shape in terms of [IS02] (in fact we know $\widehat{G}^{[4]}$ and $\widehat{G}^{[3]}$ up to isomorphism). By the main result of Section 11.7 in [IS02] $\widehat{\mathcal{F}}$ is isomorphic to the amalgam $\widetilde{\mathcal{F}}$ of maximal parabolics associated with the action of $B M$ on the $P$-geometry $\mathcal{G}(B M)$ which brings us to the final result of this section and of the whole project.

Proposition 10.29. Let $\mathcal{A}=\{G(x), G\{x, y\}\}$ be the amalgam associated with a locally projective action corresponding to case (vi) in Proposition 4.1. Then $\mathcal{A}=\mathcal{A}(B M, \Gamma(\mathcal{G}(B M))$.

Acknowledgement. The authors would like to thank: V. I. Trofimov for his fruitful cooperation on early stages of the project, Corinna Wiedorn for reading the preliminary version of the manuscript, and the anonymous referee for a number of helpful suggestions on the exposition. The project has been completed whilst the first named author was visiting the Graduate School of Mathematical Sciences at University of Tokyo in the fall of 2002. The exceptionally warm hospitality of both academic and secretarial staff is gladly acknowledged.

\section{REFERENCES}

[B78] G. Bell, On the cohomology of finite special linear groups I and II, J. Algebra, 54 (1978), 216-238 and 239-259.

[B84] R. Borcherds, The Leech lattice and other lattices, Ph.D. Thesis, Cambridge (1984).

[CP82] P. J. Cameron and C. E. Praeger, Graphs and permutation groups with projective subconstituents, J. London Math. Soc. (2), 25 (1982), 62-74.

[Ch99] K. Ching, Graphs with projective linear stabilizers, Europ. J. Combin., 20 (1999), 29-44.

[ATLAS] J. H. Conway, R. T. Curtis, S. P. Norton, R. A. Parker and R. A. Wilson, Atlas of Finite Groups, Clarendon Press, Oxford, 1985. 
[DS85] A. Delgado and B. Stellmacher, Weak $(B, N)$-pairs of rank 2, Groups and Graphs: New Results and Methods, Birkhäuser, Basel (1985), pp. 58-244.

[DM80] D. Ž. Djoković and G. L. Miller, Regular groups of automorphisms of cubic graphs, J. Combin. Theory (B), 29 (1980), 195-230.

[GAP] The GAP Group, GAP - Groups, Algorithms, and Programming, Version 4.2, Aachen, St. Andrews, 1999. (http://www-gap.dcs.st-and.ac.uk/ gap)

[Iv87] A. A. Ivanov, On 2-transitive graphs of girth 5, Europ. J. Combin., 8 (1987), 393-420.

[Iv90] A. A. Ivanov, The distance-transitive graphs admitting elations, Math. USSR Izvestiya Math., 35 (1990), 307-335.

[Iv92] A. A. Ivanov, A presentation for $J_{4}$, Proc. London Math. Soc. (3), 64 (1992), 369-396.

[Iv93] A. A. Ivanov, Graphs with projective subconstituents which contain short cycles, Surveys in Combinatorics (K. Walker, ed.), Cambridge Univ. Press, Cambridge (1993), pp. 173-190.

[Iv99] A. A. Ivanov, Geometry of Sporadic Groups I. Petersen and Tilde Geometries, Cambridge Univ. Press, Cambridge, 1999.

[Iv04] A. A. Ivanov, The Fourth Janko Group, Clarendon Press, Oxford, 2004.

[IM99] A. A. Ivanov and U. Meierfrankenfeld, A computer-free construction of $J_{4}$, J. Algebra, 219 (1999), 113-172.

[IP04] A. A. Ivanov and D. V. Pasechnik, Minimal representation of locally projective amalgams, J. London Math. Soc., 70 (2004), 142-164.

[IP98] A. A. Ivanov and C. E. Praeger, On locally projective graphs of girth 5, J. Algebraic Comb., 7 (1998), 259-283.

[IS02] A. A. Ivanov and S. V. Shpectorov, Geometry of Sporadic Groups II. Representations and Amalgams, Cambridge Univ. Press, Cambridge, 2002.

[Ja73] G. James, The modular characters of the Mathieu groups, J. Algebra, 27 (1973), $57-111$.

[JP76] W. Jones and B. Parshall, On the 1-cohomology of finite groups of Lie type, Proc. Conf. on Finite Groups (W. R. Scott and F. Gross, eds.), Acad. Press, San Diego (1976), pp. 313-327.

[K87] G. Karpilovsky, The Schur Multipliers, Oxford Univ. Press, Oxford, 1987.

[K60] A. G. Kurosh, The Theory of Groups. II, Chelsea, New York, 1960.

[MS93] U. Meierfrankenfeld and B. Stellmacher, Pushing up weak BN-pairs of rank two, Comm. Algebra, 21 (1993), 825-934.

[P94] A. Pasini, Diagram Geometries, Clarendon Press, Oxford, 1994.

[SW88] G. Stroth and R. Weiss, Modified Steinberg relations for the group $J_{4}$, Geom. Dedic., 25 (1988), 513-525.

[Tim84] F. G. Timmesfeld, Amalgams with rank 2 groups of Lie type in characteristic 2, preprint, Math. Inst. Univ. Giessen (1984).

[Tr91a] V. I. Trofimov, Stabilizers of the vertices of graphs with projective suborbits, Soviet Math. Dokl., 42 (1991), 825-828.

[Tr91b] V. I. Trofimov, More on vertex stabilizers of the symmetric graphs with pro- 
jective subconstituents, Int. Conf. Algebraic Combin., Vladimir, USSR (1991), pp. 36-37, (Russian).

[Tr92] V. I. Trofimov, Graphs with projective suborbits, Russian Acad. Sci. Izv. Math., 39 (1992), 869-894.

[Tr95a] V. I. Trofimov, Graphs with projective suborbits. Cases of small characteristics. I, Russian Acad. Sci. Izv. Math., 45 (1995), 353-398.

[Tr95b] V. I. Trofimov, Graphs with projective suborbits. Cases of small characteristics. II, Russian Acad. Sci. Izv. Math., 45 (1995), 559-576.

[Tr98] V. I. Trofimov, Graphs with projective suborbits. Exceptional cases of characteristic 2. I, Izv. Math., 62 (1998), 1221-1279.

[Tr00] V. I. Trofimov, Graphs with projective suborbits. Exceptional cases of characteristic 2. II, Izv. Math., 64 (2000), 173-192.

[Tr01] V. I. Trofimov, Graphs with projective suborbits. Exceptional cases of characteristic 2. III, Izv. Math., 65 (2001), 787-822.

[Tr03a] V. I. Trofimov, Vertex stabilizers of locally projective groups of automorphisms of graphs. A summary, Groups, combinatorics and geometry (Durham, 2001) (A. A. Ivanov, M. W. Liebeck and J. Saxl, eds.), World Sci. Publishing, River Edge, NJ (2003), pp. 313-326.

[Tr03b] V. I. Trofimov, Graphs with projective suborbits. Exceptional cases of characteristic 2. IV, Izvestiya Akad. Nauk, Mat., 67 (2003), 193-222, (Russian).

[Tu47] W. Tutte, A family of cubical graphs, Proc. Camb. Phil Soc., 43 (1947), 459-474.

[W77] R. Weiss, Über symmetrische Graphen und die projektiven Gruppen, Arch. Math., 28 (1977), 110-112.

[W78] R. Weiss, Symmetric graphs with projective subconstituents, Proc. Amer. Math. Soc., 72 (1978), 213-217.

[W79] R. Weiss, Groups with a $(B, N)$-pair and locally transitive graphs, Nagoya Math. J., 74 (1979), 1-21.

[W81] R. Weiss, s-Transitive graphs, Algebraic Methods in Graph Theory, North Holland, Amsterdam (1981), pp. 827-847.

[W82] R. Weiss, Graphs with subconstituents containing $L_{3}(p)$, Proc. Amer. Math. Soc., 85 (1982), 666-672.

\author{
A. A. Ivanov \\ Department of Mathematics \\ Imperial College \\ 180 Queen's Gate \\ London, $S W^{\text {ry } 2 B Z}$ \\ $U K$ \\ S. V. Shpectorov \\ Department of Mathematics and Statistics \\ Bowling Green State University \\ Bowling Green, OH 43403 \\ USA
}

